\title{
Measurement and Modeling of Proton-Induced Reactions on Arsenic from 35 to $200 \mathrm{MeV}$
}

\author{
Morgan B. Fox, ${ }^{1}, *$ Andrew S. Voyles,${ }^{1,2, \dagger}$ Jonathan T. Morrell, ${ }^{1}$ Lee A. Bernstein, ${ }^{1,2}$ Jon C. \\ Batchelder, ${ }^{1}$ Eva R. Birnbaum, ${ }^{3}$ Cathy S. Cutler, ${ }^{4}$ Arjan J. Koning, ${ }^{5}$ Amanda M. Lewis,${ }^{1}$ \\ Dmitri G. Medvedev, ${ }^{4}$ Francois M. Nortier, ${ }^{3}$ Ellen M. O'Brien,${ }^{3}$ and Christiaan Vermeulen ${ }^{3}$ \\ ${ }^{1}$ Department of Nuclear Engineering, University of California, Berkeley, Berkeley, California 94720, USA \\ ${ }^{2}$ Lawrence Berkeley National Laboratory, Berkeley, California 94720, USA \\ ${ }^{3}$ Los Alamos National Laboratory, Los Alamos, New Mexico 87544, USA \\ ${ }^{4}$ Brookhaven National Laboratory, Upton, New York 11973, USA \\ ${ }^{5}$ International Atomic Energy Agency, P.O. Box 100, A-1400 Vienna, Austria
}

(Dated: November 3, 2021)

\begin{abstract}
${ }^{72}$ As is a promising positron emitter for diagnostic imaging that can be employed locally using a ${ }^{72}$ Se generator. However, current reaction pathways to ${ }^{72}$ Se have insufficient nuclear data for efficient production using regional $100-200 \mathrm{MeV}$ high-intensity proton accelerators. In order to address this deficiency, stacked-target irradiations were performed at LBNL, LANL, and BNL to measure the production of the ${ }^{72} \mathrm{Se} /{ }^{72} \mathrm{As} \mathrm{PET}$ generator system via ${ }^{75} \mathrm{As}(\mathrm{p}, \mathrm{x})$ between 35 and $200 \mathrm{MeV}$. This work provides the most well-characterized excitation function for ${ }^{75} \mathrm{As}(\mathrm{p}, 4 \mathrm{n}){ }^{72}$ Se starting from threshold. Additional focus was given to report the first measurements of ${ }^{75} \mathrm{As}(\mathrm{p}, \mathrm{x}){ }^{68} \mathrm{Ge}$ and bolster an already robust production capability for the highly valuable ${ }^{68} \mathrm{Ge} /{ }^{68} \mathrm{Ga}$ PET generator. Thick target yield comparisons with prior established formation routes to both generators are made. In total, high-energy proton-induced cross sections are reported for 55 measured residual products from ${ }^{75} \mathrm{As}$, ${ }^{\text {nat }} \mathrm{Cu}$, and ${ }^{\text {nat }} \mathrm{Ti}$ targets, where the latter two materials were present as monitor foils. These results were compared with literature data as well as the default theoretical calculations of the nuclear model codes TALYS, CoH, EMPIRE, and ALICE. Reaction modeling at these energies is typically unsatisfactory due to few prior published data and many interacting physics models. Therefore, a detailed assessment of the TALYS code was performed with simultaneous parameter adjustments applied according to a standardized procedure. Particular attention was paid to the formulation of the two-component exciton model in the transition between the compound and preequilibrium regions, with a linked investigation of level density models for nuclei off of stability and their impact on modeling predictive power. This paper merges experimental work and evaluation techniques for high-energy charged-particle isotope production in an extension to an earlier study of this kind.
\end{abstract}

\section{INTRODUCTION}

Multi-hundred MeV proton accelerators are promising sites for the large scale production of medical radionuclides due to the high production rates enabled by their high-intensity beam capabilities and the long range of high-energy protons. However, the ability to reliably conduct isotope production at these accelerators and model relevant $(\mathrm{p}, \mathrm{x})$ reactions in the $100-200 \mathrm{MeV}$ range is hampered by a lack of measured data.

In the effort to improve this state of proton-induced nuclear reaction data, irradiations of arsenic have been performed. The formation of ${ }^{72} \mathrm{Se}$ and ${ }^{68} \mathrm{Ge}$ from ${ }^{75} \mathrm{As}(\mathrm{p}, \mathrm{x})$ is of particular interest for their application in diagnostic imaging as generators or "cows" for their decay daughters, ${ }^{72} \mathrm{As}$ and ${ }^{68} \mathrm{Ga}$, respectively. The present general production data for ${ }^{72} \mathrm{Se}$ at incident proton energies in the $35-200 \mathrm{MeV}$ range are scarce to non-existent. Lowenergy ${ }^{68} \mathrm{Ge}$ production data have been thoroughly assessed and already contribute to a robust production ca-

\footnotetext{
* morganbfox@berkeley.edu

$\dagger$ asvoyles@lbl.gov
}

pability set over the past decade, but extending knowledge for ${ }^{68} \mathrm{Ge}$ formation at higher-energies too should benefit its overall application. The $35-200 \mathrm{MeV}$ range is especially relevant because it is characteristic of the Los Alamos Isotope Production Facility (IPF) and the Brookhaven LINAC Isotope Producer (BLIP), where medical isotopes are created for widespread use.

${ }^{72} \mathrm{As}\left(t_{1 / 2}=26.0(1) \mathrm{h}, 87.8(22) \% \beta^{+}[1]\right)$ is a favourable positron emitting radionuclide for the imaging of slower biological processes. Its half-life makes ${ }^{72} \mathrm{As}-$ labelled radiopharmaceuticals useful for the observation of long-term metabolic processes, such as the enrichment and distribution of antibodies in tumour tissue, by positron emission tomography (PET) $[2,3]$. ${ }^{72}$ As offers the similar slow kinetic behaviour as the PET isotope ${ }^{124} \mathrm{I}$ $\left(t_{1 / 2}=4.1760(3) \mathrm{d}, 22.7(13) \% \beta^{+}[4]\right)$ but with a higher positron emission decay branch [5]. Furthermore, ${ }^{72} \mathrm{As}$ can form a promising pair with ${ }^{77} \mathrm{As}\left(t_{1 / 2}=38.83(5) \mathrm{h}\right.$, $\left.100.0(4) \% \beta^{-}, 683.2(17) \mathrm{keV} E_{\beta^{-,}, \max }[6]\right)$ for combined imaging and radiotherapy [7-9]. The high sulfur affinity of arsenic, promoting its covalent binding to thiol groups, along with the toxicity of the ${ }^{77}$ As decay spectrum, make ${ }^{72} \mathrm{As} /{ }^{77} \mathrm{As}$ an unique theranostic candidate $[8,10]$.

Current production methods for ${ }^{72} \mathrm{As}$ require a 
charged-particle beam in an accelerator setting. Existing accelerator pathways rely on ${ }^{\text {nat }} \mathrm{Ge}$ targets via the ${ }^{n a t} \mathrm{Ge}(\mathrm{p} / \mathrm{d}, \mathrm{xn}){ }^{72}$ As mechanisms in the $10-50 \mathrm{MeV}$ incident particle energy range $[3,11]$. However, these direct routes to ${ }^{72} \mathrm{As}$ constrain its use to medical centres nearby the production facility due to a half-life not appropriate for shipping or dispensing from a storage inventory. Additionally, direct production from ${ }^{\text {nat }} \mathrm{Ge}$ suffers from low thick target yields at these low incident energies and from co-production of the longer-lived radioisotopic impurities ${ }^{74,73,71}$ As $[3,11]$. Instead, recognition of the longer-lived ${ }^{72} \mathrm{Se}\left(t_{1 / 2}=8.40(8) \mathrm{d}[1]\right)$ as the parent precursor to ${ }^{72} \mathrm{As}$ creates the possibility for a ${ }^{72} \mathrm{Se} /{ }^{72} \mathrm{As}$ generator system $[2,9,11]$. Production of a generator results in ${ }^{72} \mathrm{As}$ free from other radioarsenic contaminants, on account of advantageous lifetime differences between ${ }^{72} \mathrm{Se}$ and neighboring Se nuclei, and availability restrictions at medical facilities across the globe. Measurements of a ${ }^{\text {nat }} \operatorname{Br}(\mathrm{p}, \mathrm{x})^{72} \mathrm{Se}$ production route have been undertaken but the thick target yields, even approaching $200 \mathrm{MeV}$ incident protons, are relatively low $[3,7,12,13]$. Bromine targets subjected to high power may also pose heating and/or reactivity problems $[12,13]$. The alternatively explored formation mechanism of nat $/ 70 \mathrm{Ge}(\alpha, \mathrm{xn}){ }^{72} \mathrm{Se}$ also suffers from low yields due to the short range of lower energy $\alpha$-particles combined with a relatively small $(<100 \mathrm{mb})$ production peak [14].

In contrast, proton-induced reactions on arsenic offer a potentially improved production pathway to the ${ }^{72} \mathrm{Se} /{ }^{72} \mathrm{As}$ generator system. The combination of an expected sufficient cross section over a wide energy range with a naturally monoisotopic $\left({ }^{75} \mathrm{As}\right)$, stable material that can be appropriately formed into production targets makes high-intensity, high-energy proton irradiations an enticing approach.

${ }^{68} \mathrm{Ga}\left(t_{1 / 2}=67.71(8) \mathrm{min}, 88.91(9) \% \beta^{+}[15]\right)$ has emerged as a significant short-lived positron emitter alongside the ubiquitous ${ }^{18} \mathrm{~F}$ for PET imaging in cases of general cancer, glioma, hypoxia, neuroendocrine tumours, and more $[16,17] .{ }^{68}$ Ga readily forms stable complexes with DOTA (a synthetically flexible metal chelating agent) and HBED, allowing peptides and other small molecules to be radiolabeled at high specific activities $[18,19]$. NETSpot, using ${ }^{68} \mathrm{Ga}$-DOTA, is an FDA approved PET imaging agent for neuroendocrine cancers [18]. Further, the compatibility of ${ }^{68} \mathrm{Ga}$ with a prostatespecific membrane antigen targeting ligand (PSMA-11 with HBED chelator) has led to a sought-after, highly successful PET tracer for the diagnosis of prostate cancer $[16,18,20]$. However, in a similar fashion to ${ }^{72} \mathrm{As}$, direct production by typical ${ }^{65} \mathrm{Cu}(\alpha, \mathrm{n})^{68} \mathrm{Ga}$ and ${ }^{68} \mathrm{Zn}(\mathrm{p}, \mathrm{n}){ }^{68} \mathrm{Ga}$ routes suffer from the same local accelerator production and shipping time constraints that inhibit widespread use [3]. Conversely, an indirect pathway to ${ }^{68} \mathrm{Ga}$, through its long-lived ${ }^{68} \mathrm{Ge}\left(t_{1 / 2}=270.93\right.$ (13) d [15]) parent, constitutes an effective generator system more applicable for societal application.
While the elution and separation chemistry of the ${ }^{68} \mathrm{Ge} /{ }^{68} \mathrm{Ga}$ system has been extensively developed, nuclear data for ${ }^{68} \mathrm{Ge}$ production remains partially incomplete [17]. The ${ }^{\text {nat }} \mathrm{Ga}(\mathrm{p}, \mathrm{xn}){ }^{68} \mathrm{Ge}$ route is the heavily studied, successful favourite of accelerator sites globally - particularly the prominent facilities of IPF, BLIP, and iThemba labs - but data only reaches up to $100 \mathrm{MeV}$. Other ${ }^{69} \mathrm{Ga}(\mathrm{p}, \mathrm{xn})^{68} \mathrm{Ge}$, ${ }^{\text {nat }} \mathrm{Ge}(\mathrm{p}, \mathrm{pxn}){ }^{68} \mathrm{Ge}$, and ${ }^{66} \mathrm{Zn}(\alpha, 2 \mathrm{n}){ }^{68} \mathrm{Ge}$ low-energy pathways have been explored but are less ideal due to excitation functions that peak in the 15-35 MeV range, which may be suboptimal for thick target yields, and present target manufacturing and purity concerns $[17,19]$. Studying proton-induced reactions on arsenic gives a chance to strengthen the community's total understanding of ${ }^{68} \mathrm{Ge} /{ }^{68} \mathrm{Ga}$ formation.

In this work, proton-induced nuclear reaction data for ${ }^{75}$ As were measured for energies $35-200 \mathrm{MeV}$ using the stacked-target method as part of the DOE Isotope Program's Tri-laboratory Effort in Nuclear Data (TREND) between Lawrence Berkeley National Laboratory (LBNL), Los Alamos National Laboratory (LANL), and Brookhaven National Laboratory (BNL) [21]. We report the first cross section measurements for ${ }^{75} \mathrm{As}(\mathrm{p}, \mathrm{x}){ }^{68} \mathrm{Ge}$ and the most well-characterized excitation function of ${ }^{75} \mathrm{As}(\mathrm{p}, 4 \mathrm{n}){ }^{72} \mathrm{Se}$ to-date. Thick target yields are additionally calculated from the measured excitation functions and compared to established formation routes for the generator radionuclides to better inform accelerator facilities of optimal production parameters.

This stacked-target work has further provided 53 other high-energy $(\mathrm{p}, \mathrm{x})$ production cross section datasets for residual nuclei stemming from ${ }^{75} \mathrm{As}$, ${ }^{\text {nat }} \mathrm{Cu}$, and ${ }^{\text {nat }} \mathrm{Ti}$ targets.

These extensive measurements were also used to assess the predictions of multiple nuclear reaction codes. The standardized fitting procedure for reaction model parameters and pre-equilibrium adjustments developed in Fox et al. [21] was applied to the arsenic data, with an investigative focus to check if the proposed exciton model trends are seen.

In addition to studying pre-equilibrium, the fitting procedure provided insight into the appropriate level density models for a swath of nuclei. A discussion of the impact of level density knowledge on modeling predictive power is presented with a reflection of the limitations imposed on creating recommended high-energy charged-particle data.

The combination of experimental measurement and evaluation study presented in this work creates data with immediate application while contributing to an increasingly prioritized future need for high-energy modeling in the nuclear data community [22]. 


\section{EXPERIMENTAL METHODS AND MATERIALS}

This work is an outcome of the same set of experimental irradiations and activations performed for Fox et al. [21] but gives a focus to the analysis and interpretation of arsenic, titanium, and copper target foils not previously discussed. Charged-particle stacked-target irradiations were carried out at the 88-Inch Cyclotron at LBNL for proton energies of $E_{p}<55 \mathrm{MeV}$, at IPF at LANL for $50<E_{p}<100 \mathrm{MeV}$, and at BLIP at BNL for $100<E_{p}<200 \mathrm{MeV}$.

The stacked-target technique is a typical methodology for charged-particle irradiations to simultaneously measure multiple high-fidelity energy-separated cross section values per reaction channel. A stacked-target includes thin foils of a target of interest in combination with thick degraders and monitor foils. The degraders selectively reduce the primary beam energy throughout the stack while the monitor foils can be used to characterize the evolving beam properties as it propagates through the targets. Detailed explanations of the technique can be read in [21, 23-27].

\section{A. Stacked-Target Design}

Individual stacks were created for each irradiation at each experimental site. The three stacks differed slightly in composition according to the physical constraints of each site's irradiation geometry and as a function of expected residual radionuclide production based on beam current and energy parameters.

\section{LBNL Stack and Irradiation}

The 88-Inch Cyclotron stack consisted of $25 \mu \mathrm{m}{ }^{\text {nat }} \mathrm{Cu}$ foils (99.95\%, CU000420, Goodfellow Metals, Coraopolis, PA 15108-9302, USA) and thin metallic ${ }^{75}$ As layers electroplated onto $10 \mu \mathrm{m}$ or $25 \mu \mathrm{m}$ nat $\mathrm{Ti}$ foil backings (99.6\%, TI000213/TI000290, Goodfellow Metals).

Nine copper and titanium foils each were cut into $2.5 \mathrm{~cm} \times 2.5 \mathrm{~cm}$ squares and characterized by taking four length and width measurements using a digital caliper (Mitutoyo America Corp.) and four thickness measurements taken at different locations using a digital micrometer (Mitutoyo America Corp.). Each foil was also massed multiple times using an analytical balance at $0.1 \mathrm{mg}$ precision after being cleaned with isopropyl alcohol. The characterization of the approximately $2.25 \mathrm{~cm}$ diameter arsenic depositions onto titanium, pictured in Figure 1, was a more intensive process involving particle transmission and neutron activation analysis. These details and the description of the associated electroplating creation process are given in Voyles et al. [28], while the resulting thickness and areal density values can be seen in Table I.
All targets were then sealed using DuPont Kapton polyimide film tape of either $43.2 \mu \mathrm{m}$ of silicone adhesive on $25.4 \mu \mathrm{m}$ of polyimide backing (total nominal $7.77 \mathrm{mg} / \mathrm{cm}^{2}$ ) or $43.2 \mu \mathrm{m}$ of silicone adhesive on $50.8 \mu \mathrm{m}$ of polyimide backing (total nominal $11.89 \mathrm{mg} / \mathrm{cm}^{2}$ ). The encapsulated foils were mounted to the center of hollow $5.7 \mathrm{~cm} \times 5.7 \mathrm{~cm}$ aluminum frames. The frames protected the foils during handling and centered them in the beam pipe after the stack was fully arranged in the target box seen in Figure 2.

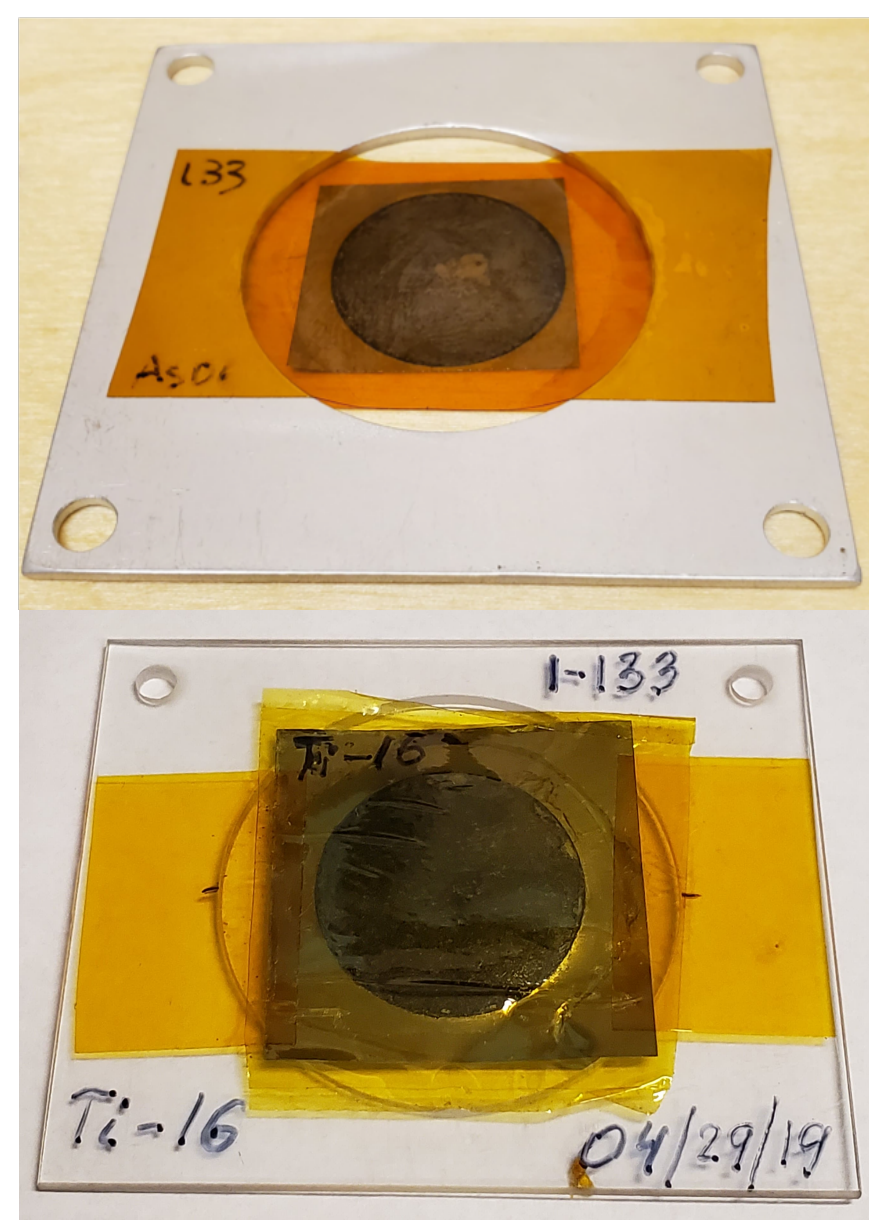

FIG. 1. View of individual electroplated arsenic depositions on titanium backings within Kapton seals. The top target is sampled from the LBNL stack and is pictured after proton irradiation, where slight bubbling in the Kapton seal exists as a result of beam heating. The bottom target is part of the BNL stack prior to proton irradiation. 


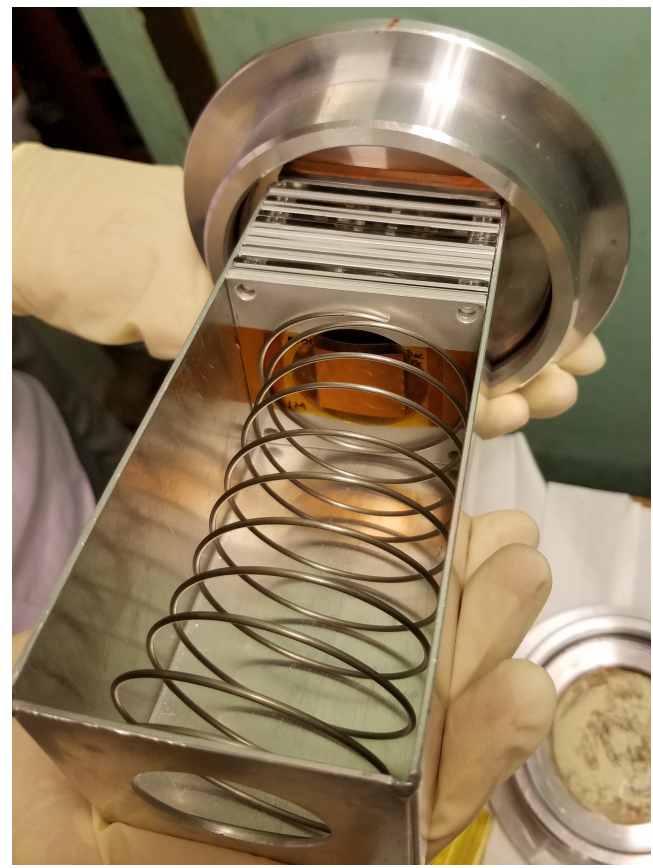

FIG. 2. A top view of the assembled LBNL target stack prior to loading into the cyclotron beam pipe. The beam is first incident on the front facing copper target shown in the photo, as described in Table I.

Multiple aluminum degraders were characterized in the same manner as the copper foils and included in the stack to yield nine different beam energy "compartments" for cross section measurements. One copper foil and one electroplated arsenic foil were placed into each of the nine compartments in the target box. Stainless steel plates (approximately $100 \mathrm{mg} / \mathrm{cm}^{2}$ ) were placed near the front and back of the stack for post-irradiation dose mapping using radiochromic film (Gafchromic EBT3) in order to examine the spatial profile of the beam entering and exiting the stack. The full detailed target stack ordering and properties for the LBNL irradiation are given in Table I.

The stack was irradiated at the 88-Inch Cyclotron for 3884 seconds with a nominal $192 \mathrm{nA} \mathrm{H}^{+}$beam. The total collected charge of the beam was measured using a current integrator connected to the electrically-isolated target holder, which was used to determine that the beam current was stable over the duration of the experiment. The mean beam energy extracted was $55.4 \mathrm{MeV}$ with an approximately $1 \%$ uncertainty.

\section{LANL Stack and Irradiation}

The LANL stack included copper, niobium, aluminum, and electroplated arsenic targets. The stack composition is described in detail in Fox et al. [21], where characterization procedures were very similar to the LBNL setup. A summary of the stack is provided in this paper in Table VII (see Appendix A). The stack was irradiated for 7203 seconds with an $\mathrm{H}^{+}$beam of $100 \mathrm{nA}$ nominal current.
TABLE I. Target stack design for irradiation at the 88-Inch Cyclotron. The proton beam initially hits the $\mathrm{Cu}-\mathrm{SN} 1$ target and is subsequently transported through the rest of the shown stack order. The thickness and areal density measurements are prior to any application of the variance minimization techniques described in this work.

\begin{tabular}{|c|c|c|c|}
\hline Target Layer & $\begin{array}{c}\text { Thickness } \\
{[\mu \mathrm{m}]}\end{array}$ & $\begin{array}{c}\text { Areal } \\
\text { Density } \\
{\left[\mathrm{mg} / \mathrm{cm}^{2}\right]}\end{array}$ & $\begin{array}{c}\text { Areal } \\
\text { Density } \\
\text { Uncertainty } \\
{[\%]}\end{array}$ \\
\hline $\mathrm{Cu}-\mathrm{SN} 1$ & 24.81 & 22.23 & 0.33 \\
\hline As-SN1 & 3.24 & 1.85 & 9.8 \\
\hline Ti-SN1 & 25.00 & 11.265 & 1.0 \\
\hline SS Profile Monitor & 130.0 & 100.12 & 0.07 \\
\hline Al Degrader E1 & 253.0 & 68.31 & 0.10 \\
\hline Al Degrader E2 & 252.7 & 68.24 & 0.10 \\
\hline $\mathrm{Cu}-\mathrm{SN} 2$ & 24.88 & 22.29 & 0.08 \\
\hline As-SN2 & 1.69 & 0.97 & 9.9 \\
\hline Ti-SN2 & 25.00 & 11.265 & 1.0 \\
\hline Al Degrader D1 & 674.2 & 174.44 & 0.05 \\
\hline $\mathrm{Cu}-\mathrm{SN} 3$ & 24.88 & 22.29 & 0.06 \\
\hline As-SN3 & 1.81 & 1.04 & 9.9 \\
\hline Ti-SN3 & 25.00 & 11.265 & 1.0 \\
\hline Al Degrader D2 & 664.5 & 174.87 & 0.06 \\
\hline $\mathrm{Cu}-\mathrm{SN} 4$ & 24.87 & 22.28 & 0.04 \\
\hline As-SN4 & 2.22 & 1.27 & 10 \\
\hline Ti-SN4 & 25.00 & 11.265 & 1.0 \\
\hline Al Degrader E3 & 253.1 & 68.35 & 0.10 \\
\hline $\mathrm{Cu}-\mathrm{SN} 5$ & 24.97 & 22.37 & 0.06 \\
\hline As-SN5 & 1.95 & 1.12 & 9.9 \\
\hline Ti-SN5 & 25.00 & 11.265 & 1.0 \\
\hline Al Degrader F1 & 181.5 & 46.91 & 0.12 \\
\hline Al Degrader F2 & 192.2 & 48.97 & 0.14 \\
\hline $\mathrm{Cu}-\mathrm{SN} 6$ & 24.85 & 22.27 & 0.09 \\
\hline As-SN6 & 1.30 & 0.74 & 11 \\
\hline Ti-SN6 & 25.00 & 11.265 & 1.0 \\
\hline Al Degrader E4 & 252.9 & 68.29 & 0.10 \\
\hline $\mathrm{Cu}-\mathrm{SN} 7$ & 24.67 & 22.11 & 0.39 \\
\hline As-SN7 & 2.36 & 1.35 & 8.9 \\
\hline Ti-SN7 & 10.00 & 4.506 & 1.0 \\
\hline Al Degrader C1 & 970.0 & 261.48 & 0.03 \\
\hline $\mathrm{Cu}-\mathrm{SN} 8$ & 24.80 & 22.22 & 0.06 \\
\hline As-SN8 & 0.94 & 0.54 & 9.7 \\
\hline Ti-SN8 & 25.00 & 11.265 & 1.0 \\
\hline Al Degrader E5 & 252.7 & 68.24 & 0.10 \\
\hline $\mathrm{Cu}-\mathrm{SN} 9$ & 24.90 & 22.31 & 0.10 \\
\hline As-SN9 & 0.57 & 0.32 & 10 \\
\hline Ti-SN9 & 25.00 & 11.265 & 1.0 \\
\hline SS Profile Monitor & 130.0 & 100.48 & 0.07 \\
\hline
\end{tabular}


The beam current, measured using an inductive pickup, remained stable under these conditions for the duration of the irradiation. The mean beam energy extracted was $100.16 \mathrm{MeV}$ with an approximately $0.1 \%$ uncertainty.

\section{BNL Stack and Irradiation}

The BNL stack was composed of copper, niobium, and electroplated arsenic targets. The exact specifications of the stack are given in Fox et al. [21] and a summary can be seen in Table VIII (see Appendix A). The stack was irradiated for 3609 seconds with an $\mathrm{H}^{+}$beam of $200 \mathrm{nA}$ nominal current. The beam current during operation was recorded using toroidal beam transformers and shown to remain stable under these conditions for the duration of the irradiation. The mean beam energy extracted was $200 \mathrm{MeV}$ with an approximately $0.2 \%$ uncertainty [7].

\section{B. Gamma Spectroscopy and Measurement of Foil Activities}

\section{1. $L B N L$}

The gamma spectroscopy at the 88-Inch Cyclotron utilized an ORTEC GMX series (model GMX-50220S) High-Purity Germanium (HPGe) detector and seven ORTEC IDM-200-VTM HPGe detectors. The GMX is a nitrogen-cooled coaxial n-type HPGe with a $0.5 \mathrm{~mm}$ beryllium window, and a $64.9 \mathrm{~mm}$ diameter, $57.8 \mathrm{~mm}$ long crystal. The IDMs are mechanically-cooled coaxial p-type HPGes with single, large-area $85 \mathrm{~mm}$ diameter $\times$ $30 \mathrm{~mm}$ length crystals and built-in spectroscopy electronics. The energy and absolute photopeak efficiency of the GMX and IDMs were calibrated using standard ${ }^{133} \mathrm{Ba}$, ${ }^{137} \mathrm{Cs}$, and ${ }^{152} \mathrm{Eu}$ sources. The efficiency model used in this work is the physical model presented by Gallagher and Cipolla [29].

Foil activity data was first collected from counts beginning approximately 45 minutes after the end-ofbombardment (EoB) and removal of the target stack from the beamline. The copper and electroplated arsenic foils were initially cycled through multiple $5-30$ minute counts on the GMX during the 24 hours immediately following the irradiation. The counting distances from the GMX detector face were varied from $80 \mathrm{~cm}$ to $15 \mathrm{~cm}$ during this period subject to dead time constraints. Each electroplated arsenic foil was then transferred to an individual IDM detector where counts were collected in 1 hour intervals at a $10 \mathrm{~cm}$ distance from the IDM face over the next three weeks. The repeated counts of each foil helped to establish consistent decay curves for residual nuclides and reduce uncertainty in the spectroscopy analysis, particularly aiding in the determination of longerlived products. Final 12-24 hour counts for the copper foils were captured on the GMX near the end of the three week period to record appropriate statistics for long-lived monitor channels.

The radiochromic film, developed by the stainless steel plates, showed that an $\approx 1 \mathrm{~cm}$ diameter proton beam was centered on the stack foils and properly inscribed within the size-limiting borders of the arsenic deposits throughout the stack.

\section{2. $L A N L$}

The LANL experiment used a series of GEM and IDM HPGe detectors. The foil counting at LANL followed a similar cycling routine to LBNL, with counting times ranging from 10 minutes during the first hours after EoB to upwards of 8 hours over the course of 6 weeks after the irradiation for the stack's 40 total targets. The LANL counting scheme is given explicitly in Fox et al. [21]. Notably, the electroplated arsenic targets of the LANL stack were shipped to LBNL in order to perform multi-week long counts with the LBNL GMX to better capture the ${ }^{68} \mathrm{Ge}$ signal, which remained weak in the longest of the LANL counts.

\section{3. $B N L$}

The BNL gamma spectroscopy setup incorporated two EURISYS MESURES 2 Fold Segmented "Clover" detectors in addition to one GMX and two GEM detectors. Foils were cycled in front of the many detectors for repeated short counts of 30 minutes or less during the first 24 hours after EoB. Data collection at BNL continued with multi-hour target counts for an additional day before the targets were shipped back to LBNL, arriving within two weeks after EoB. The LBNL GMX was used for multi-day to week-long counts of the copper, electroplated arsenic, and niobium foils over the course of the next $2+$ months.

Further details of the BLIP activation and spectroscopy is provided in Fox et al. [21].

\section{Activation Analysis}

The UC Berkeley code package Curie [30] was used to analyze the collected gamma spectra from each irradiation. Decay curves for observed residual products were constructed from the count data with appropriate timing, efficiency, and attenuation corrections. EoB activities $A_{0}$ were then determined by fitting decay curves to the applicable Bateman equations [21, 23, 24]. A sample gamma-ray spectrum from an electroplated arsenic target is given in Figure 3.

Independent, $(i), A_{0}$ results were determined from decay curve fits where decay contributions from any precursors of a residual product could be distinguished or where no parent decay in-feeding existed. In cases where 


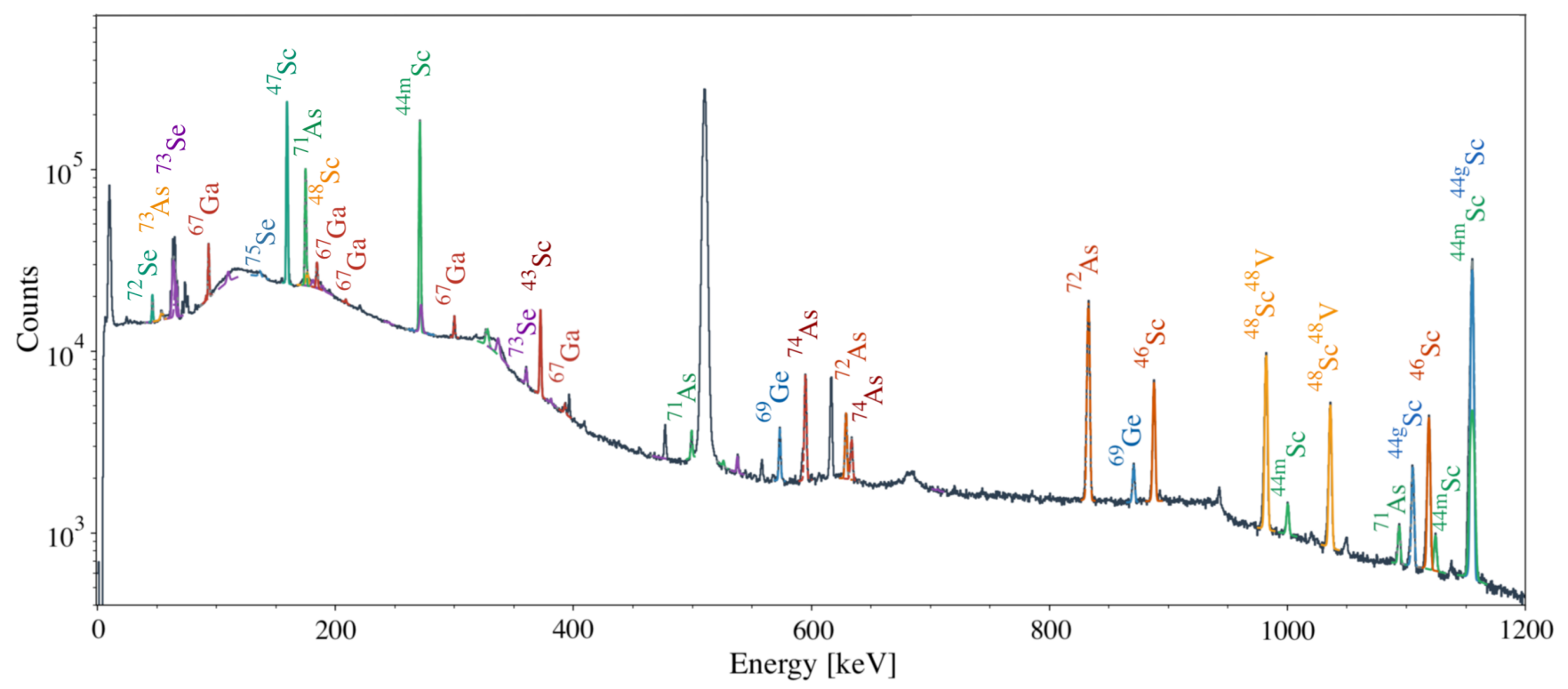

FIG. 3. Example gamma-ray spectrum from the induced activation of an electroplated arsenic target in the LANL stack at approximately $E_{p}=91 \mathrm{MeV}$. The spectrum was taken slightly beyond 2 days after EoB and the smooth fits to the peaks of interest shown are produced by the Curie package [31].

precursor contributions could not be distinguished, either due to timing or decay property limitations, cumulative, $(c), A_{0}$ values for a residual product within a decay chain were instead calculated.

The total uncertainties in the determined EoB activities had contributions from fitted peak areas, evaluated half-lives and gamma intensities, regression parameters, and detector efficiency calibrations. Each contribution to the total uncertainty was assumed to be independent and was added in quadrature. The impact of calculated $A_{0}$ uncertainties on final cross section results is detailed in Section IID.

\section{Stack Current and Energy Properties}

The proton beam energy and current at each target in a given stack was determined by monitor foil activation data, Curie's Andersen \& Ziegler-based Monte Carlo particle transport code, and a "variance minimization" approach, following the established methodology presented in Voyles et al. [23], Morrell et al. [24], and Graves et al. [25].

The ${ }^{\text {nat }} \mathrm{Ti}(\mathrm{p}, \mathrm{x}){ }^{48} \mathrm{~V},{ }^{46} \mathrm{Sc}$ and ${ }^{\text {nat }} \mathrm{Cu}(\mathrm{p}, \mathrm{x}){ }^{63,62} \mathrm{Zn},{ }^{58} \mathrm{Co}$ monitor reactions, taken from the IAEA-recommended data reference for charged-particle reactions [32], were used for the LBNL beam characterization. The results after variance minimization are shown in Figure 4 with plotted weighted averages of all the monitor reaction fluence predictions in each stack compartment. The weighted averages account for data and measurement correlations between the monitor reaction channels at each position in the stack and were used to create the uncertainty-weighted linear fit, also included in Figure 4 [33]. The fit is a global model applied due to the observed flat fluence depletion and provides an interpolation for the fluence and energy of each individual target of interest in the stack. This optimized linear model after variance minimization shows an approximately constant $207 \mathrm{nAh}$ fluence throughout the LBNL stack.

Further details of the monitor foil calculations, variance minimization approach, and energy determinations for the LBNL experiment can be reviewed in Appendix B. An in-depth discussion of this same beam characterization procedure for the LANL and BNL stacks is provided in Fox et al. [21]. Recall that this work and Fox et al. [21] are outcomes of the same set of target stacks and irradiations meaning that the LANL and BNL fluence results and energy assignments from Fox et al. [21] are identically applied here.

The final deduced energy assignments, with associated uncertainties, for targets in all three stacks are provided in Tables II, III, and IV. 


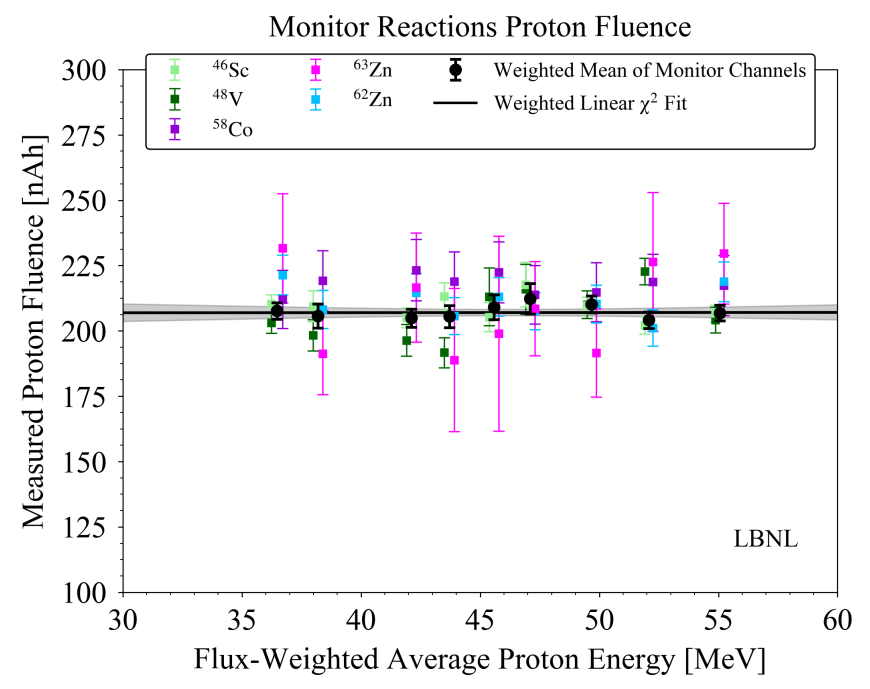

FIG. 4. Plot of the proton beam fluence measured by monitor reactions in the LBNL stack following adjustments made by the variance minimization technique.

\section{Cross Section Determination}

Cross sections for observed products in this work were calculated from the typical activation formula,

$$
\sigma=\frac{A_{0}}{I_{p}\left(\rho_{N} \Delta r\right)\left(1-e^{-\lambda t_{i r r}}\right)},
$$

where $I_{p}$ is the beam current in protons per second at a given foil in a stack, $\rho_{N} \Delta r$ is the relevant foil's areal number density, $\lambda$ is the decay constant for the observed residual product of interest, and $t_{i r r}$ is the beam-on irradiation time.

Measured ${ }^{75} \mathrm{As}(\mathrm{p}, \mathrm{x})$ cross sections are reported in Table II for ${ }^{75,73,72} \mathrm{Se},{ }^{74-70} \mathrm{As},{ }^{72,68-66} \mathrm{Ga},{ }^{69,68,66} \mathrm{Ge}$, ${ }^{69 m}, 65 \mathrm{Zn}$, and ${ }^{60,58-56} \mathrm{Co}$.

${ }^{\text {nat }} \mathrm{Cu}(\mathrm{p}, \mathrm{x})$ production cross sections for ${ }^{65,63,62} \mathrm{Zn}$, ${ }^{64,61,60} \mathrm{Cu}, \quad{ }^{60,57-55} \mathrm{Co}, \quad{ }^{59} \mathrm{Fe}, \quad{ }^{57,56} \mathrm{Ni}, \quad{ }^{56,54,52} \mathrm{Mn}$, ${ }^{51,49,48} \mathrm{Cr},{ }^{48} \mathrm{~V}$, and ${ }^{47,46,44 \mathrm{~m}} \mathrm{Sc}$ are given in Table III.

${ }^{\text {nat }} \mathrm{Ti}(\mathrm{p}, \mathrm{x})$ experimental cross section results for ${ }^{48} \mathrm{~V}$,

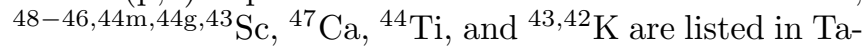
ble IV.

In Tables II III, and IV, the cross sections for residual products are marked as either independent, $(i)$, or cumulative, $(c)$, referencing the distinction discussed in Section II B 4 surrounding decay chains.

The final uncertainty contributions to the cross section measurements include uncertainties in evaluated decay constants $(0.02-1.0 \%)$, foil areal density measurements (0.05-11\%), proton current determination calculated from monitor fluence measurements and variance minimization (1.1-3.4\%), and $A_{0}$ quantification that accounts for efficiency uncertainty in addition to other factors listed in Section II B 4 (1.5-14\%). These contributions were added in quadrature to give uncertainty in the final cross section results at the $3.5-17 \%$ level.

\section{RESULTS AND DISCUSSION}

The measured data from select reactions of particular interest to the medical applications community or for nuclear reaction modeling purposes are discussed in detail below. Plots of all other reported cross sections are given in Appendix C (Figures 19-69).

The experimentally extracted cross sections are compared with the predictions of nuclear reaction modeling codes TALYS-1.95 [34], CoH-3.5.3 [35], EMPIRE-3.2.3 [36], and ALICE-20 [37], each using default settings and parameters. A discussion of these default conditions and assumptions is provided in Fox et al. [21]. Comparisons with the TENDL-2019 library [38] are also made. Additionally, the cross section measurements in this work are compared to the existing body of literature data, retrieved from EXFOR [7, 23-25, 39-79].

\section{A. ${ }^{75} \mathrm{As}(\mathbf{p}, \mathbf{4 n}){ }^{72}$ Se Cross Section}

${ }^{72}$ Se decays $100 \%$ by electron capture to the first $1^{+}$ excited state in ${ }^{72} \mathrm{As}$. This leaves a $45.89 \mathrm{keV}\left(I_{\gamma}=\right.$ $57.2(4) \%) \gamma$-ray as the only direct detectable signature of ${ }^{72} \mathrm{Se}$ formation from the HPGe equipment used in this work. However, ${ }^{72} \mathrm{Se}$ production could additionally be quantified using the ${ }^{72}$ As decay gamma-rays after ${ }^{72} \mathrm{Se} /{ }^{72} \mathrm{As}$ were in secular equilibrium at least 11 days after EoB. The results from each measurement method were seen to be very comparable but only the secular equilibrium values were recorded, and plotted in Figure 5 , due to comparatively reduced uncertainties.

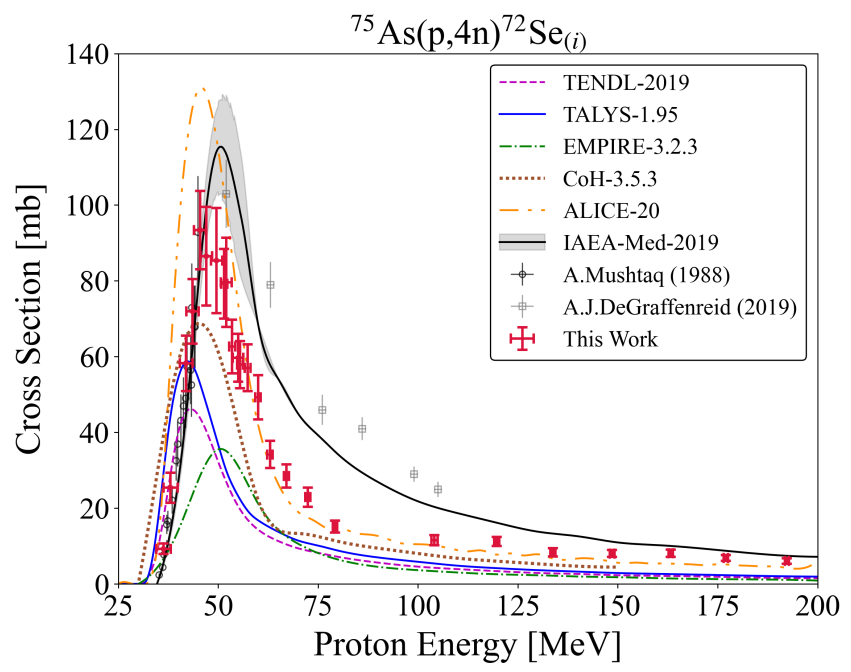

FIG. 5. Experimental and theoretical cross sections for ${ }^{72} \mathrm{Se}$ production, peaking near $90 \mathrm{mb}$ around $50 \mathrm{MeV}$.

Only two prior experimental datasets partially measured this excitation function. The Mushtaq et al. [39] results cover the low energy production from threshold towards the maximum of the compound peak near $50 \mathrm{MeV}$ and agree well with the measurements of this work. The 
TABLE II. Summary of arsenic cross sections measured in this work. Subscripts $(i)$ and $(c)$ indicate independent and cumulative cross sections, respectively. Uncertainties are listed in the least significant digit, that is, $49.5(14) \mathrm{MeV}$ means $49.5 \pm 1.4 \mathrm{MeV}$. Stack ID specifies which irradiation each measurement belongs to - Stack "BR" designates the Brookhaven irradiation, Stack "LA" designates the Los Alamos irradiation, and Stack "LB" designates the Lawrence Berkeley irradiation.

\begin{tabular}{|c|c|c|c|c|c|c|c|c|c|}
\hline \multicolumn{10}{|c|}{${ }^{75}$ As $(\mathbf{p}, \mathbf{x})$ Production Cross Sections [mb] } \\
\hline$E_{\mathrm{p}}[\mathrm{MeV}]$ & $192.28(49)$ & $177.01(51)$ & $163.21(54)$ & $148.55(58)$ & $133.75(62)$ & $119.66(67)$ & $104.09(73)$ & $91.09(51)$ & $79.19(56)$ \\
\hline Stack ID & $\mathrm{BR}$ & BR & $\mathrm{BR}$ & $\mathrm{BR}$ & $\mathrm{BR}$ & $\mathrm{BR}$ & $\mathrm{BR}$ & LA & LA \\
\hline${ }^{56} \mathrm{Co}_{(c)}$ & $0.823(98)$ & $0.337(34)$ & $0.581(64)$ & $0.436(48)$ & $0.169(28)$ & - & - & - & - \\
\hline${ }^{57} \mathrm{Co}_{(c)}$ & $3.04(46)$ & $1.36(18)$ & $2.03(28)$ & $1.68(25)$ & $0.51(17)$ & - & - & - & - \\
\hline${ }^{58} \mathrm{Co}_{(i)}$ & $3.62(80)$ & - & $2.81(33)$ & $2.25(26)$ & $0.84(11)$ & $0.32(24)$ & $0.07(8)$ & - & - \\
\hline${ }^{60} \mathrm{Co}_{(i)}$ & $8.8(11)$ & $1.89(20)$ & $1.70(20)$ & $1.06(14)$ & - & - & - & - & - \\
\hline${ }^{65} \mathrm{Zn}_{(c)}$ & $45.8(77)$ & $47.6(58)$ & $47.4(63)$ & $35.1(43)$ & $29.2(38)$ & $31.4(38)$ & $10.8(15)$ & - & - \\
\hline${ }^{66} \mathrm{Ga}_{(c)}$ & $11.1(66)$ & $24.9(66)$ & $31(17)$ & $24.1(98)$ & $16.0(42)$ & - & $14.6(39)$ & $5.43(89)$ & $5.33(95)$ \\
\hline${ }^{66} \mathrm{Ge}_{(c)}$ & - & - & - & $1.15(49)$ & $1.18(22)$ & - & - & - & - \\
\hline${ }^{67} \mathrm{Ga}_{(c)}$ & $39.1(46)$ & $44.8(45)$ & $43.2(47)$ & $42.1(43)$ & $38.6(49)$ & $36.7(36)$ & $35.0(39)$ & $20.6(19)$ & $25.5(24)$ \\
\hline${ }^{68} \mathrm{Ga}_{(i)}$ & $41.7(83)$ & $39.2(62)$ & $41.3(58)$ & $40.7(69)$ & $35.5(53)$ & $42.8(55)$ & $39.5(52)$ & - & - \\
\hline${ }^{68} \mathrm{Ge}_{(c)}$ & $30.7(46)$ & $26.9(30)$ & $26.4(32)$ & $22.8(27)$ & $21.9(30)$ & $20.3(23)$ & $13.0(16)$ & $11.1(22)$ & $24.1(41)$ \\
\hline${ }^{69 m} \mathrm{Zn}_{(i)}$ & $1.24(19)$ & $1.38(22)$ & $1.38(17)$ & $1.26(14)$ & $1.02(24)$ & $1.29(13)$ & $0.75(13)$ & - & - \\
\hline${ }^{69} \mathrm{Ge}_{(c)}$ & $36.9(43)$ & $40.5(43)$ & $41.6(50)$ & $37.0(42)$ & $36.9(49)$ & $42.5(44)$ & $35.0(39)$ & $19.8(20)$ & $16.2(16)$ \\
\hline${ }^{70} \mathrm{As}_{(c)}$ & $15.9(18)$ & $16.4(17)$ & $17.7(19)$ & $16.4(17)$ & $17.2(21)$ & $23.2(23)$ & $27.1(28)$ & $36.9(39)$ & 43.7 (45) \\
\hline${ }^{71} \mathrm{As}_{(c)}$ & $40.0(45)$ & $49.2(51)$ & $55.2(64)$ & $55.8(60)$ & $64.3(79)$ & $76.2(76)$ & $73.4(75)$ & - & $91.8(85)$ \\
\hline${ }^{72} \mathrm{Ga}_{(c)}$ & - & - & - & $1.39(57)$ & $3.07(95)$ & $1.89(68)$ & $3.38(82)$ & $2.20(29)$ & $2.31(49)$ \\
\hline${ }^{72} \mathrm{As}_{(i)}$ & $70.3(77)$ & $82.6(82)$ & $80.3(90)$ & $89.2(94)$ & $97(12)$ & $122(12)$ & $116(12)$ & - & 108.8 \\
\hline${ }^{72} \mathrm{Se}_{(i)}$ & $6.12(72)$ & $6.90(75)$ & $8.12(94)$ & $8.09(89)$ & $8.4(11)$ & $11.2(12)$ & $11.6(13)$ & - & $15.2(16)$ \\
\hline${ }^{73} \mathrm{As}_{(i)}$ & $95(17)$ & $125(19)$ & $138(24)$ & $128(24)$ & $138(26)$ & $166(28)$ & $172(31)$ & $180(42)$ & $174(24)$ \\
\hline${ }^{73} \mathrm{Se}_{(c)}$ & $11.9(15)$ & $14.0(16)$ & $14.8(17)$ & $15.6(18)$ & $18.0(24)$ & $23.0(25)$ & $23.5(27)$ & $22.8(29)$ & $25.7(35)$ \\
\hline${ }^{74} \mathrm{As}_{(i)}$ & $98(11)$ & $112(12)$ & $113(16)$ & 118 (14) & $124(18)$ & $138(14)$ & $148(18)$ & - & $123(12)$ \\
\hline${ }^{75} \mathrm{Se}_{(i)}$ & $5.55(59)$ & $6.65(63)$ & $7.47(79)$ & $6.80(69)$ & $7.44(89)$ & $9.23(88)$ & $9.48(95)$ & $6.08(52)$ & $10.10(87)$ \\
\hline$E_{\mathrm{p}}[\mathrm{MeV}]$ & $72.39(60)$ & $67.00(64)$ & $62.92(67)$ & $59.93(69)$ & $57.31(72)$ & $55.42(74)$ & $54.9(13)$ & $53.46(76)$ & $52.0(14)$ \\
\hline Stack ID & LA & LA & LA & LA & LA & LA & LB & LA & LB \\
\hline${ }^{66} \mathrm{Ga}_{(c)}$ & $2.88(64)$ & - & - & - & - & - & - & - & - \\
\hline${ }^{67} \mathrm{Ga}_{(c)}$ & $16.4(18)$ & $6.2(10)$ & $2.32(77)$ & $1.00(78)$ & $0.91(74)$ & - & - & - & - \\
\hline${ }^{68} \mathrm{Ge}_{(c)}$ & $41.4(72)$ & $39.2(69)$ & $31.1(54)$ & $14.1(20)$ & - & - & - & - & - \\
\hline${ }^{69} \mathrm{Ge}_{(c)}$ & $17.6(19)$ & $20.6(22)$ & $25.6(26)$ & $34.5(40)$ & $39.4(42)$ & $37.4(40)$ & $41.5(44)$ & $39.6(45)$ & $35.8(39)$ \\
\hline${ }^{70} \mathrm{As}_{(c)}$ & $33.1(40)$ & - & $2.3(10)$ & - & - & - & - & $2.3(16)$ & - \\
\hline${ }^{71} \mathrm{As}_{(c)}$ & $131(13)$ & $143(14)$ & $130(12)$ & $128(14)$ & $103(11)$ & $74.9(77)$ & $63.9(65)$ & $53.6(61)$ & $32.3(34)$ \\
\hline${ }^{72} \mathrm{Ga}_{(c)}$ & $1.72(51)$ & $2.25(47)$ & - & $1.26(47)$ & - & $1.31(48)$ & - & $1.03(34)$ & - \\
\hline${ }^{72} \mathrm{As}_{(i)}$ & $146(14)$ & $169(17)$ & $188(18)$ & $238(26)$ & $262(26)$ & $249(24)$ & $277(28)$ & $266(28)$ & $246(25)$ \\
\hline${ }^{72} \mathrm{Se}_{(i)}$ & $23.0(25)$ & $28.5(31)$ & $34.2(36)$ & $49.3(58)$ & $57.1(62)$ & $57.9(62)$ & $59.8(63)$ & $62.7(71)$ & $80(12)$ \\
\hline${ }^{73} \mathrm{As}_{(i)}$ & $229(32)$ & $244(35)$ & $252(35)$ & $323(47)$ & $325(47)$ & $282(40)$ & - & $346(60)$ & $320(53)$ \\
\hline${ }^{73} \mathrm{Se}_{(c)}$ & $37.4(48)$ & $39.0(55)$ & $45.2(55)$ & $54.2(81)$ & $62.1(82)$ & $57.0(80)$ & $60.1(69)$ & $65.4(89)$ & $65.4(76)$ \\
\hline${ }^{74} \mathrm{As}_{(i)}$ & $153(16)$ & $158(17)$ & $157(16)$ & $186(21)$ & $185(19)$ & $169(17)$ & $188(20)$ & $170(19)$ & $182(19)$ \\
\hline${ }^{75} \mathrm{Se}_{(i)}$ & $13.2(12)$ & $14.2(13)$ & $14.4(13)$ & $16.9(18)$ & $17.7(17)$ & $16.2(15)$ & $15.2(16)$ & $16.9(18)$ & $16.1(18)$ \\
\hline$E_{\mathrm{p}}[\mathrm{MeV}]$ & $51.44(78)$ & $49.5(14)$ & $47.0(15)$ & $45.4(15)$ & $43.6(16)$ & $41.9(16)$ & $38.0(17)$ & $36.3(18)$ & \\
\hline Stack ID & LA & LB & LB & LB & LB & LB & LB & LB & \\
\hline${ }^{69} \mathrm{Ge}_{(c)}$ & $40.6(48)$ & $31.5(34)$ & $27.6(31)$ & $17.6(20)$ & $13.0(16)$ & $12.0(12)$ & - & - & \\
\hline${ }^{71} \mathrm{As}_{(c)}$ & $39.6(48)$ & $17.4(19)$ & $9.7(11)$ & $6.44(77)$ & $8.2(11)$ & $3.46(39)$ & - & - & \\
\hline${ }^{72} \mathrm{Ga}_{(c)}$ & - & - & - & - & - & - & $0.21(13)$ & - & \\
\hline${ }^{72} \mathrm{As}_{(i)}$ & $280(30)$ & $226(23)$ & $219(22)$ & $207(22)$ & - & $131(12)$ & $73.8(85)$ & $41.9(55)$ & \\
\hline${ }^{72} \mathrm{Se}_{(i)}$ & $79.3(92)$ & $85(14)$ & $87(13)$ & $93(10)$ & $72.0(85)$ & $58.3(73)$ & $25.4(40)$ & $9.3(14)$ & \\
\hline${ }^{73} \mathrm{As}_{(i)}$ & $345(52)$ & $359(65)$ & $469(79)$ & $460(69)$ & $570(100)$ & $587(85)$ & $680(110)$ & $600(94)$ & \\
\hline${ }^{73} \mathrm{Se}_{(c)}$ & $80(12)$ & $69.6(79)$ & $91(10)$ & $92(11)$ & $114(14)$ & $205(21)$ & $235(26)$ & $307(37)$ & \\
\hline${ }^{74} \mathrm{As}_{(i)}$ & $186(22)$ & $181(19)$ & $194(21)$ & $193(21)$ & - & $234(23)$ & $218(24)$ & $239(27)$ & \\
\hline${ }^{75} \mathrm{Se}_{(i)}$ & $18.0(19)$ & $17.8(20)$ & $17.0(18)$ & $17.2(20)$ & $21.8(35)$ & $23.8(23)$ & $25.0(31)$ & $26.5(39)$ & \\
\hline
\end{tabular}


TABLE III. Summary of copper cross sections measured in this work. Subscripts $(i)$ and $(c)$ indicate independent and cumulative cross sections, respectively. Uncertainties are listed in the least significant digit, that is, 90.94 (52) $\mathrm{MeV}$ means $90.94 \pm 0.52 \mathrm{MeV}$. Stack ID specifies which irradiation each measurement belongs to - Stack "BR" designates the Brookhaven irradiation, Stack "LA" designates the Los Alamos irradiation, and Stack "LB" designates the Lawrence Berkeley irradiation.

\begin{tabular}{|c|c|c|c|c|c|c|c|c|c|c|}
\hline \multicolumn{11}{|c|}{${ }^{{ }^{n a t}} \mathbf{C u}(\mathrm{p}, \mathrm{x})$ Production Cross Sections [mb] } \\
\hline$E_{\mathrm{p}}[\mathrm{MeV}]$ & $192.54(49)$ & $177.28(52)$ & $163.49(54)$ & $148.86(58)$ & $134.08(62)$ & $120.02(67)$ & $104.49(74)$ & $90.94(52)$ & $79.03(57)$ & $72.22(61)$ \\
\hline Stack ID & $\mathrm{BR}$ & $\mathrm{BR}$ & $\mathrm{BR}$ & $\mathrm{BR}$ & $\mathrm{BR}$ & $\mathrm{BR}$ & $\mathrm{BR}$ & LA & LA & LA \\
\hline$\overline{{ }^{44 m} \mathrm{Sc}_{(i)}}$ & $0.289(12)$ & $0.1338(63)$ & $0.0784(85)$ & $0.0444(40)$ & - & - & - & - & - & - \\
\hline${ }^{46} \mathrm{Sc}_{(i)}$ & $0.572(21)$ & $0.335(11)$ & $0.2381(65)$ & $0.1065(59)$ & $0.0616(30)$ & $0.0375(24)$ & - & - & - & - \\
\hline${ }^{47} \mathrm{Sc}_{(c)}$ & $0.261(46)$ & $0.182(31)$ & $0.218(26)$ & - & - & - & - & - & - & - \\
\hline${ }^{48} \mathrm{~V}_{(c)}$ & $2.346(84)$ & $1.560(47)$ & $1.162(30)$ & $0.689(29)$ & $0.499(15)$ & $0.298(45)$ & - & - & - & - \\
\hline${ }^{48} \mathrm{Cr}_{(c)}$ & 0.0707 & $0.0437(19)$ & $0.0263(27)$ & $0.0207(11)$ & - & - & - & - & - & - \\
\hline${ }^{49} \mathrm{Cr}_{(c)}$ & $0.943(67)$ & $0.624(60)$ & $0.411(46)$ & - & - & - & - & - & - & - \\
\hline${ }^{51} \mathrm{Cr}_{(c)}$ & $11.59(42)$ & $9.79(29)$ & $8.44(21)$ & $6.46(26)$ & $5.33(13)$ & $4.35(13)$ & $1.676(68)$ & $1.220(61)$ & $0.427(49)$ & 0.469 \\
\hline${ }^{52} \mathrm{Mn}_{(c)}$ & $5.34(19)$ & $4.72(14)$ & $4.22(11)$ & $3.34(12)$ & $2.733(70)$ & $1.934(59)$ & $1.727(70)$ & $1.759(67)$ & $0.509(22)$ & 0.1008 \\
\hline${ }^{54} \mathrm{Mn}_{(i)}$ & $16.26(59)$ & $15.72(48)$ & $14.88(38)$ & $13.4(12)$ & $12.48(31)$ & $11.05(32)$ & $7.30(27)$ & $6.63(23)$ & $3.87(15)$ & $3.86(17)$ \\
\hline${ }^{55} \mathrm{Co}_{(c)}$ & $2.04(11)$ & $2.12(11)$ & $1.995(97)$ & $2.06(10)$ & $1.813(91)$ & $1.679(90)$ & $1.77(10)$ & $2.50(18)$ & $1.43(11)$ & $0.647(60)$ \\
\hline${ }^{56} \mathrm{Mn}_{(c)}$ & $2.52(15)$ & $2.54(15)$ & $2.46(14)$ & $2.18(13)$ & $2.07(13)$ & $1.85(11)$ & $1.40(10)$ & $1.186(57)$ & $1.106(54)$ & $0.927(43)$ \\
\hline${ }^{56} \mathrm{Co}_{(i)}$ & $12.50(43)$ & $12.65(35)$ & $12.57(29)$ & $13.18(34)$ & $12.29(27)$ & $11.55(31)$ & $10.51(37)$ & $10.31(44)$ & $12.12(49)$ & $12.68(56)$ \\
\hline${ }^{56} \mathrm{Ni}_{(c)}$ & $0.072(59)$ & $0.089(12)$ & $0.116(12)$ & 0.105 & $0.131(15)$ & $0.093(15)$ & - & $0.0884(75)$ & $0.1103(82)$ & $0.1070(81)$ \\
\hline${ }^{57} \mathrm{Co}_{(c)}$ & $43.0(35)$ & $42.3(14)$ & $43.1(12)$ & $43.6(11)$ & $44.5(12)$ & $44.7(14)$ & $42.2(16)$ & $44.7(14)$ & $37.7(11)$ & 36.9 (11) \\
\hline${ }^{57} \mathrm{Ni}_{(c)}$ & $1.687(85)$ & $1.787(66)$ & $1.820(61)$ & $1.776(57)$ & - & - & - & $1.76(11)$ & $1.286(83)$ & $1.391(89)$ \\
\hline${ }^{59} \mathrm{Fe}_{(c)}$ & $1.180(51)$ & $1.209(45)$ & $1.189(40)$ & $1.100(50)$ & $1.097(36)$ & $1.045(38)$ & $0.923(40)$ & $0.931(33)$ & $0.867(29)$ & $0.817(29)$ \\
\hline${ }^{60} \mathrm{Co}_{(i)}$ & $11.72(47)$ & $13.66(61)$ & $13.73(48)$ & $11.28(55)$ & 12.41 & $12.24(38)$ & 12.01 & $14.21(42)$ & $12.50(37)$ & $11.48(36)$ \\
\hline${ }^{60} \mathrm{Cu}_{(c)}$ & $8.01(42)$ & $9.37(48)$ & $10.75(57)$ & $13.77(77)$ & $11.4(10)$ & $15.1(14)$ & $16.5(19)$ & $16.87(75)$ & $16.0(10)$ & $17.38(90)$ \\
\hline${ }^{61} \mathrm{Cu}_{(c)}$ & $29.9(16)$ & $33.2(16)$ & $36.4(17)$ & $39.0(17)$ & $42.9(19)$ & $46.6(22)$ & $55.7(29)$ & $60.6(30)$ & $54.3(29)$ & $72.5(35)$ \\
\hline${ }^{62} \mathrm{Zn}_{(i)}$ & $1.71(11)$ & $2.16(13)$ & $1.86(12)$ & $2.44(14)$ & $2.39(15)$ & $3.42(19)$ & $3.26(21)$ & - & - & - \\
\hline${ }^{63} \mathrm{Zn}_{(i)}$ & $3.52(34)$ & $4.32(45)$ & $5.25(63)$ & $6.05(87)$ & $5.52(97)$ & $5.73(93)$ & - & $8.40(52)$ & $10.90(71)$ & $12.98(78)$ \\
\hline${ }^{64} \mathrm{Cu}_{(i)}$ & $26.3(15)$ & $31.7(18)$ & $30.8(34)$ & 35.1 (18) & $36.6(35)$ & $40.7(22)$ & 44.7 (39) & $52.0(57)$ & $40.4(55)$ & $50.3(51)$ \\
\hline${ }^{65} \mathrm{Zn}_{(i)}$ & $1.13(26)$ & $1.52(20)$ & $1.61(16)$ & $1.53(11)$ & $1.938(83)$ & $2.200(78)$ & $2.69(11)$ & $2.868(95)$ & $3.257(95)$ & $3.68(11)$ \\
\hline$E_{\mathrm{p}}[\mathrm{MeV}]$ & $66.81(65)$ & $62.73(68)$ & $59.73(71)$ & $57.11(73)$ & $55.21(75)$ & $55.2(13)$ & $53.24(77)$ & $52.2(14)$ & $51.22(80)$ & $49.9(14)$ \\
\hline Stack ID & LA & LA & LA & LA & LA & $\mathrm{LB}$ & LA & LB & LA & LB \\
\hline${ }^{51} \mathrm{Cr}_{(c)}$ & $0.512(37)$ & $0.409(38)$ & $0.328(33)$ & $0.278(29)$ & - & - & - & - & - & - \\
\hline${ }^{54} \mathrm{Mn}_{(i)}$ & $4.70(17)$ & $4.95(33)$ & $4.70(27)$ & $4.10(20)$ & $3.41(15)$ & $3.58(14)$ & $2.65(11)$ & $2.31(13)$ & $1.848(74)$ & $1.25(10)$ \\
\hline${ }^{55} \mathrm{Co}_{(c)}$ & $0.169(22)$ & $0.077(15)$ & $0.060(20)$ & $0.043(12)$ & $0.0394(92)$ & $0.0127(40)$ & - & - & $0.0162(69)$ & - \\
\hline${ }^{56} \mathrm{Mn}_{(c)}$ & $0.644(33)$ & $0.460(25)$ & $0.243(18)$ & $0.171(15)$ & $0.161(14)$ & $0.101(13)$ & 0.089 (11) & - & $0.0541(91)$ & - \\
\hline${ }^{56} \mathrm{Co}_{(i)}$ & $10.95(46)$ & $7.66(32)$ & $4.47(18)$ & $2.405(99)$ & $1.272(62)$ & - & $0.713(39)$ & - & $0.373(57)$ & - \\
\hline${ }^{56} \mathrm{Ni}_{(c)}$ & $0.0837(61)$ & 0.0518 & $0.0330(28)$ & $0.0144(28)$ & $0.0082(26)$ & - & $0.0076(22)$ & - & $0.0043(13)$ & - \\
\hline${ }^{57} \mathrm{Co}_{(c)}$ & $42.4(13)$ & $50.0(21)$ & $55.9(23)$ & $59.5(26)$ & $58.7(26)$ & $64.6(50)$ & $58.0(25)$ & $55.6(12)$ & $54.8(24)$ & $49.9(10)$ \\
\hline${ }^{57} \mathrm{Ni}_{(c)}$ & $1.78(11)$ & $2.32(10)$ & $2.61(12)$ & $2.73(12)$ & $2.60(12)$ & 2.608 (99) & $2.38(11)$ & $1.942(62)$ & $1.985(90)$ & $1.502(47)$ \\
\hline${ }^{59} \mathrm{Fe}_{(c)}$ & $0.775(27)$ & $0.690(29)$ & $0.618(26)$ & $0.516(22)$ & $0.419(19)$ & - & $0.322(14)$ & - & $0.227(10)$ & - \\
\hline${ }^{60} \mathrm{Co}_{(i)}$ & $11.68(36)$ & $12.22(49)$ & $12.15(47)$ & $11.60(46)$ & $10.88(51)$ & $10.34(41)$ & $10.77(49)$ & $10.04(39)$ & $10.28(40)$ & $9.53(36)$ \\
\hline${ }^{60} \mathrm{Cu}_{(c)}$ & $18.6(15)$ & $27.2(23)$ & - & $26.1(38)$ & $26.4(29)$ & $30.1(27)$ & - & $33.6(25)$ & - & $29.5(25)$ \\
\hline${ }^{61} \mathrm{Cu}_{(c)}$ & $82.8(39)$ & $89.7(42)$ & - & $91.9(44)$ & $94.2(45)$ & $91.1(42)$ & $93.6(45)$ & $94.0(42)$ & $97.5(47)$ & $103.7(45)$ \\
\hline${ }^{63} \mathrm{Zn}_{(i)}$ & $12.29(88)$ & $14.0(11)$ & $16.3(13)$ & $17.5(16)$ & $17.9(20)$ & - & - & - & - & - \\
\hline${ }^{64} \mathrm{Cu}_{(i)}$ & $61.7(60)$ & $51.4(56)$ & $63.0(62)$ & $66.6(66)$ & $59.7(56)$ & $60.7(30)$ & $55.4(59)$ & $56.1(28)$ & $62.7(62)$ & $57.3(32)$ \\
\hline${ }^{65} \mathrm{Zn}_{(i)}$ & $4.05(11)$ & $4.21(20)$ & $4.39(19)$ & $4.66(21)$ & $4.79(24)$ & $4.53(23)$ & $5.32(28)$ & $4.65(25)$ & $5.30(26)$ & $5.51(28)$ \\
\hline$E_{\mathrm{p}}[\mathrm{MeV}]$ & $47.3(15)$ & $45.8(15)$ & $43.9(16)$ & $42.3(16)$ & $38.4(17)$ & $36.7(18)$ & & & & \\
\hline Stack ID & LB & LB & LB & LB & LB & LB & & & & \\
\hline${ }^{54} \mathrm{Mn}_{(i)}$ & $0.533(15)$ & $0.160(43)$ & $0.091(29)$ & $0.020(18)$ & $0.076(30)$ & $0.092(28)$ & & & & \\
\hline${ }^{57} \mathrm{Co}_{(c)}$ & $36.36(68)$ & $29.27(61)$ & $17.91(41)$ & $11.09(29)$ & $1.446(96)$ & $0.398(34)$ & & & & \\
\hline${ }^{57} \mathrm{Ni}_{(c)}$ & $0.909(32)$ & $0.634(26)$ & $0.309(19)$ & $0.1257(93)$ & - & - & & & & \\
\hline${ }^{60} \mathrm{Co}_{(i)}$ & $8.78(16)$ & $7.72(31)$ & $7.12(31)$ & $5.95(32)$ & $4.95(27)$ & $4.35(24)$ & & & & \\
\hline${ }^{60} \mathrm{Cu}_{(c)}$ & $19.3(22)$ & $9.3(21)$ & $5.5(17)$ & 4.7 (18) & - & - & & & & \\
\hline${ }^{61} \mathrm{Cu}_{(c)}$ & $112.6(48)$ & $125.9(54)$ & $137.7(59)$ & $156.9(67)$ & $179.8(77)$ & $187.4(82)$ & & & & \\
\hline${ }^{64} \mathrm{Cu}_{(i)}$ & $58.1(31)$ & 66.5 & $59.7(30)$ & 64.9 & 63.1 & $74.4(36)$ & & & & \\
\hline${ }^{65} \mathrm{Zn}_{(i)}$ & $5.57(12)$ & $5.50(26)$ & $6.19(27)$ & $6.32(29)$ & $6.97(30)$ & $7.33(34)$ & & & & \\
\hline
\end{tabular}


TABLE IV. Summary of titanium cross sections measured in this work. Subscripts $(i)$ and $(c)$ indicate independent and cumulative cross sections, respectively. Uncertainties are listed in the least significant digit, that is, 72.34 (61) MeV means $72.34 \pm 0.61 \mathrm{MeV}$. Stack ID specifies which irradiation each measurement belongs to - Stack "BR" designates the Brookhaven irradiation, Stack "LA" designates the Los Alamos irradiation, and Stack "LB" designates the Lawrence Berkeley irradiation.

\begin{tabular}{|c|c|c|c|c|c|c|c|c|c|}
\hline & & & ${ }^{\text {nat }} \mathbf{T i}(\mathbf{p}, \mathbf{x})$ & Production & Cross Secti & ons $[\mathrm{mb}]$ & & & \\
\hline$E_{\mathrm{p}}[\mathrm{MeV}]$ & $192.26(49)$ & $176.99(51)$ & $163.18(54)$ & $148.52(58)$ & $133.72(62)$ & $119.63(67)$ & $104.05(74)$ & $91.05(51)$ & $79.15(57)$ \\
\hline Stack ID & $\mathrm{BR}$ & $\mathrm{BR}$ & $\mathrm{BR}$ & $\mathrm{BR}$ & $\mathrm{BR}$ & $\mathrm{BR}$ & $\mathrm{BR}$ & LA & LA \\
\hline${ }^{42} \mathrm{~K}_{(i)}$ & $7.54(78)$ & $6.45(70)$ & $6.83(66)$ & $6.34(67)$ & $6.92(64)$ & $5.56(62)$ & $6.10(88)$ & $6.73(47)$ & $6.48(43)$ \\
\hline${ }^{43} \mathrm{~K}_{(c)}$ & $2.62(10)$ & $2.493(90)$ & $2.84(11)$ & $2.34(10)$ & $2.23(10)$ & $2.116(83)$ & $1.95(13)$ & $1.830(58)$ & $1.349(45)$ \\
\hline${ }^{43} \mathrm{Sc}_{(c)}$ & $16.5(11)$ & $15.9(11)$ & $12.8(22)$ & $15.17(95)$ & $17.1(11)$ & $20.0(14)$ & - & $22.8(19)$ & $15.0(16)$ \\
\hline${ }^{44 \mathrm{~g} \mathrm{Sc}_{(i)}}$ & $25.1(13)$ & $26.5(16)$ & $27.9(13)$ & $28.49(97)$ & $28.5(10)$ & $31.5(17)$ & $31.7(15)$ & $32.2(19)$ & $39.3(22)$ \\
\hline${ }^{44 \mathrm{~m}} \mathrm{Sc}_{(i)}$ & $11.46(44)$ & $11.88(40)$ & $12.71(37)$ & $13.43(39)$ & $14.47(80)$ & $14.82(85)$ & $19.1(16)$ & $21.34(72)$ & $22.29(73)$ \\
\hline${ }^{44} \mathrm{Ti}_{(c)}$ & 2.7 (18) & $2.8(11)$ & $3.3(10)$ & $4.37(42)$ & $3.3(17)$ & $4.55(49)$ & - & - & - \\
\hline${ }^{46} \mathrm{Sc}_{(i)}$ & $34.0(13)$ & $36.1(12)$ & $38.2(11)$ & $39.3(10)$ & $39.3(11)$ & $40.9(13)$ & $41.5(16)$ & $42.1(15)$ & $42.3(13)$ \\
\hline${ }^{47} \mathrm{Ca}(c)$ & $0.167(22)$ & $0.187(27)$ & $0.168(30)$ & $0.158(39)$ & - & - & - & - & - \\
\hline${ }^{47} \mathrm{Sc}_{(i)}$ & $25.7(21)$ & $25.84(98)$ & $26.53(87)$ & $26.82(84)$ & $26.2(13)$ & $26.70(97)$ & $26.0(28)$ & $23.5(12)$ & $22.4(11)$ \\
\hline${ }^{48} \mathrm{Sc}_{(i)}$ & $2.31(15)$ & $2.35(16)$ & $1.85(44)$ & $1.88(13)$ & $2.53(31)$ & - & $2.65(42)$ & $2.45(13)$ & $2.35(13)$ \\
\hline${ }^{48} \mathrm{~V}_{(i)}$ & $3.62(13)$ & $4.11(13)$ & $4.16(12)$ & $4.86(12)$ & $5.60(17)$ & $6.24(20)$ & $7.06(28)$ & - & - \\
\hline$E_{\mathrm{p}}[\mathrm{MeV}]$ & $72.34(61)$ & $66.95(64)$ & $62.87(67)$ & $59.88(70)$ & $57.26(72)$ & $55.36(74)$ & $54.9(13)$ & $53.40(76)$ & $51.9(14)$ \\
\hline Stack ID & LA & LA & LA & LA & LA & LA & LB & LA & LB \\
\hline${ }^{42} \mathrm{~K}_{(i)}$ & $6.94(49)$ & $7.32(51)$ & $6.57(43)$ & $5.62(37)$ & $4.30(31)$ & $3.23(23)$ & $2.86(20)$ & $2.77(22)$ & $1.72(11)$ \\
\hline${ }^{43} \mathrm{~K}_{(c)}$ & $1.295(46)$ & $1.358(44)$ & $1.339(45)$ & $1.425(48)$ & $1.408(48)$ & $1.532(51)$ & $1.400(34)$ & $1.439(54)$ & $1.333(28)$ \\
\hline${ }^{43} \mathrm{Sc}_{(c)}$ & $15.4(14)$ & $13.9(15)$ & $15.2(14)$ & $15.7(17)$ & $17.9(17)$ & $18.6(20)$ & $14.22(84)$ & $19.0(17)$ & $15.83(88)$ \\
\hline${ }^{44 \mathrm{~g} \mathrm{Sc}_{(i)}}$ & $35.4(23)$ & - & 30.4 (17) & 27.7 (17) & $21.3(27)$ & $24.65(78)$ & $21.3(12)$ & $22.22(71)$ & $22.2(12)$ \\
\hline${ }^{44 \mathrm{~m}} \mathrm{Sc}_{(i)}$ & $23.03(78)$ & $21.13(69)$ & $18.18(61)$ & $15.97(53)$ & $14.23(47)$ & $13.52(45)$ & $12.02(24)$ & $12.79(42)$ & $10.48(22)$ \\
\hline${ }^{46} \mathrm{Sc}_{(i)}$ & $44.8(16)$ & $48.0(16)$ & $50.0(17)$ & $51.8(21)$ & $53.2(19)$ & $55.5(21)$ & - & $55.3(18)$ & - \\
\hline${ }^{47} \mathrm{Sc}_{(i)}$ & $23.2(11)$ & $23.7(11)$ & $23.8(11)$ & $23.9(11)$ & $23.6(11)$ & $23.5(11)$ & $20.82(65)$ & $22.7(11)$ & $19.08(64)$ \\
\hline${ }^{48} \mathrm{Sc}_{(i)}$ & $2.33(12)$ & $2.30(12)$ & $2.28(13)$ & $2.18(15)$ & $2.131(87)$ & $2.02(13)$ & $1.649(85)$ & $2.01(12)$ & $1.596(44)$ \\
\hline$E_{\mathrm{p}}[\mathrm{MeV}]$ & $51.39(79)$ & $49.5(14)$ & $46.9(15)$ & $45.4(15)$ & $43.5(16)$ & $41.9(16)$ & 38.0 (17) & $36.2(18)$ & \\
\hline Stack ID & LA & LB & LB & LB & LB & LB & LB & LB & \\
\hline${ }^{42} \mathrm{~K}_{(i)}$ & $1.67(16)$ & $1.151(90)$ & $0.786(67)$ & $0.670(80)$ & $0.571(55)$ & - & 0.378 & - & \\
\hline${ }^{43} \mathrm{~K}_{(c)}$ & $1.394(52)$ & $1.169(25)$ & 0.863 (19) & 0.645 (17) & $0.473(12)$ & - & $0.1122(65)$ & - & \\
\hline${ }^{43} \mathrm{Sc}_{(c)}$ & $20.6(22)$ & $16.12(90)$ & $16.32(91)$ & $15.80(92)$ & - & $13.18(85)$ & $9.38(58)$ & $6.54(42)$ & \\
\hline${ }^{44 \mathrm{gSc}_{(i)}}$ & $23.24(72)$ & $19.4(12)$ & $22.7(15)$ & $22.3(16)$ & $23.8(17)$ & $25.1(11)$ & $29.8(11)$ & $33.73(97)$ & \\
\hline${ }^{44} \mathrm{mSc}_{(i)}$ & $12.86(42)$ & $11.54(26)$ & $12.00(28)$ & $11.61(22)$ & $12.16(28)$ & $12.24(26)$ & $15.03(39)$ & $13.45(32)$ & \\
\hline${ }^{46} \mathrm{Sc}_{(i)}$ & $59.7(21)$ & - & - & - & - & - & - & - & \\
\hline${ }^{47} \mathrm{Sc}_{(i)}$ & $23.0(11)$ & $20.41(87)$ & $20.89(92)$ & $19.87(50)$ & $20.37(72)$ & $19.16(57)$ & $23.62(93)$ & $22.35(70)$ & \\
\hline${ }^{48} \mathrm{Sc}_{(i)}$ & $2.01(12)$ & $1.836(70)$ & $1.809(51)$ & $1.684(91)$ & $1.627(49)$ & $1.370(52)$ & $1.296(62)$ & $1.003(70)$ & \\
\hline
\end{tabular}

second prior experimental dataset from DeGraffenreid et al. [7] covers a broader higher-energy portion of the excitation function between 52-105 MeV. A large discrepancy exists between the DeGraffenreid et al. [7] data and the values reported here. This difference is most evident for the cross section above $60 \mathrm{MeV}$ where our measurements demonstrate a much more constrained "bellshape" for the compound peak with a pre-equilibrium "tail" that decreases in magnitude quicker than expressed by DeGraffenreid et al. [7]. These differences are possibly partly a function of the contrasting experimental methodologies between this work and DeGraffenreid et al. [7]. DeGraffenreid et al. [7] did not use a stackedtarget technique, but instead used multiple irradiations with thicker GaAs wafer targets, a much larger beam current, and analysis by chemical dissolution of the targets with subsequent radioassays on an HPGe using solution aliquots.

The TALYS, CoH, and ALICE reaction codes, along with the TENDL evaluation, demonstrate a similar shape though all but ALICE underpredict the compound peak cross section magnitude. Incorrect compound peak energy centroids are a pervasive error among all the calculations for this channel, generally as a function of the 
codes' poor threshold predictions. TENDL perhaps best matches the experimental threshold and rising edge behaviour of the excitation function but its incorrect magnitude, on account of misestimated competition with adjacent channels, muddles some of the comparison of the evaluation to the data.

In general, the variation in peak centroid location between the codes is typical and is a function of the differing pre-equilibrium calculations. Small differences between pre-equilibrium models in the codes can amplify the impact caused by particles emitted in pre-equilibrium that carry a significant amount of energy, which ultimately alter which compound nucleus is formed at a given incident energy [24]. Consequently, the improper pre-equilibrium tail modeling among TALYS, CoH, EMPIRE, and TENDL is noteworthy because it is an error that will propagate to the thresholding and rising edge behaviour in residual products that are energetically downstream of this $(\mathrm{p}, 4 \mathrm{n})$ channel.

Moreover, EMPIRE performs worst among the codes likely on account of these incorrect pre-equilibrium results for residual products closer in mass to the target nucleus. In this ${ }^{72}$ Se channel, the errors in EMPIRE manifest as an estimated rising edge with a much too small of a slope and the largest magnitude underprediction.

The production cross section of ${ }^{72}$ Se has also been evaluated as part of an IAEA coordinated research project (IAEA-Med-2019) focused on the recommendation of data for medical radionuclides, and in specific, diagnostic positron emitters [3]. The DeGraffenreid et al. [7] data were not avaialble at the time of the IAEA evaluation and though the IAEA prediction reaches a similar peak to DeGraffenreid et al. [7], which is above the peak predicted in this work, the IAEA recommendation does not support the very broad compound peak.

It is worth reflecting that these ${ }^{72}$ Se production results, i.e., the proper characterization of an excitation function from threshold to $200 \mathrm{MeV}$ where little prior data existed, are emblematic of the overall TREND endeavour.

\section{B. ${ }^{75} \mathrm{As}(\mathbf{p}, \mathbf{x}){ }^{68} \mathrm{Ge}$ Cross Section}

The results reported here represent the first measurement of this channel. The ${ }^{68} \mathrm{Ge}$ production cross section proved difficult to quantify in this work due to its long half-life $\left(t_{1 / 2}=270.93(13) \mathrm{d}[15]\right)$ and the lack of gamma-ray emissions. ${ }^{68} \mathrm{Ge}$ decays $100 \%$ by electron capture directly to the ground state of ${ }^{68} \mathrm{Ga}$. As a result, it was necessary to rely on the still weak, but strongest available, $1077.34 \mathrm{keV}\left(I_{\gamma}=3.22(3) \%\right) \gamma$-ray from the decay of ${ }^{68} \mathrm{Ga}$ to measure the ${ }^{68} \mathrm{Ge}$ formation cross section [80]. ${ }^{68} \mathrm{Ga}$ is short-lived with a 67.71 (8) min half-life and it quickly falls into secular equilibrium with ${ }^{68} \mathrm{Ge}$ [15]. Therefore, all $1077.34 \mathrm{keV}$ emissions measured in the arsenic target spectra taken months after the irradiation dates were solely attributable to the decay of the initial cumulative ${ }^{68} \mathrm{Ge}$ population. Multi-week-long counts were required to achieve reasonable statistics for the $1077.34 \mathrm{keV}$ signal.

The ensuing measured ${ }^{75} \mathrm{As}(\mathrm{p}, \mathrm{x}){ }^{68} \mathrm{Ge}$ excitation function is given in Figure 6. No cross sections were extracted from the LBNL irradiation or the rear-end of the LANL stack as the incident proton energies were below or too near threshold for measurable ${ }^{68} \mathrm{Ge}$ production. The given excitation function in Figure 6 is the first measurement of ${ }^{68} \mathrm{Ge}$ formation from arsenic up to $200 \mathrm{MeV}$. The excitation function shows a peak of approximately $42 \mathrm{mb}$ at $72 \mathrm{MeV}$ due to the ${ }^{75} \mathrm{As}(\mathrm{p}, \alpha 4 \mathrm{n}){ }^{68} \mathrm{Ge}$ pathway and a high-energy increasing pre-equilibrium tail from formation mechanisms where $\alpha$-particle emission is replaced by $2 \mathrm{p} 2 \mathrm{n}$. The cross section is additionally impacted by the shape of the ${ }^{68}$ As excitation function since the given result is cumulative.

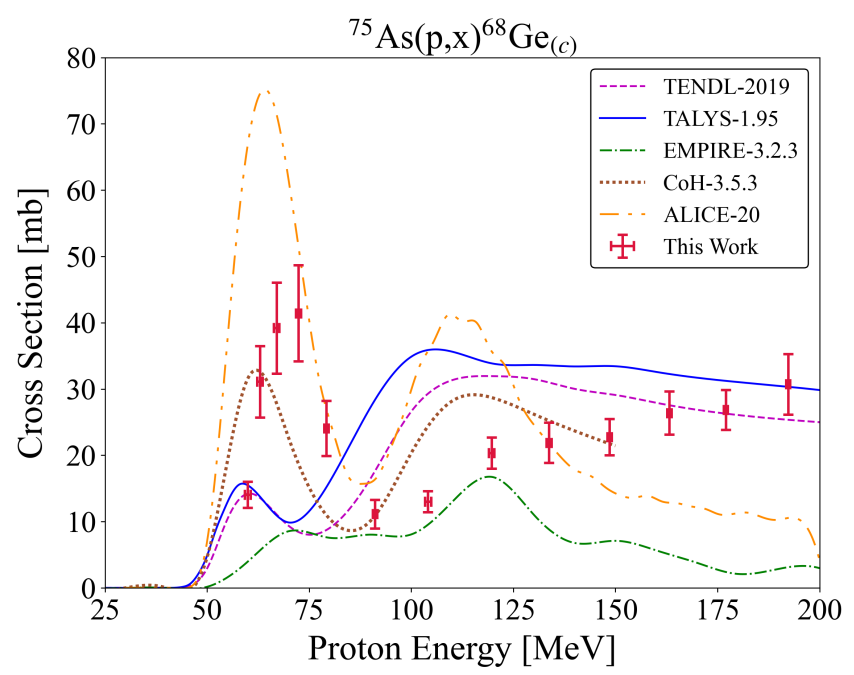

FIG. 6. Experimental and theoretical cross sections for ${ }^{68} \mathrm{Ge}$ production, peaking near $42 \mathrm{mb}$ around $72 \mathrm{MeV}$.

Interestingly, EMPIRE's overprediction of the compound peak energy centroid for ${ }^{72} \mathrm{Se}$ production versus all other codes (Figure 5) is also seen for the ${ }^{68} \mathrm{Ge}$ excitation function except it is a fairly accurate representation of reality in Figure 6. However, this energy comparison is the endpoint of EMPIRE's accuracy as its excitation function shape and magnitude are markedly incorrect.

ALICE continues to overestimate the compound peak magnitude and it even incorrectly predicts a higherenergy second compound peak rather than a preequilibrium tail. $\mathrm{CoH}$ performs similarly to ALICE but at a more correct magnitude albeit at a shifted centroid energy of near $10 \mathrm{MeV}$ below the experimental data. Both TALYS and TENDL correctly demonstrate a significant pre-equilibrium tail with an approximately correct shape, similar to $\mathrm{CoH}$, but the relative magnitudes between their peaks and tails are erroneous.

It is important to temper expectations for the predictive power of these codes in calculating the ${ }^{68} \mathrm{Ge}$ production seen here since this is a cumulative result. Note that in the cumulative cases of this work, the code calculations 
shown include necessary summing of decay precursor contributions. ${ }^{68} \mathrm{Ge}$ therefore requires calculation contributions from three residual products and ultimately only makes up a minor $\approx 5 \%$ of the total non-elastic cross section, which creates a difficult predictive case.

\section{C. ${ }^{75} \operatorname{As}(\mathbf{p}, 3 \mathbf{3 n}){ }^{73}$ Se Cross Section}

The ${ }^{75} \mathrm{As}(\mathrm{p}, 3 \mathrm{n}){ }^{73} \mathrm{Se}$ excitation function is the most well-characterized residual product channel in the prior literature data. The measured cross sections extracted from the LBNL and LANL irradiations are shown in Figure 7 to agree very well with these existing results. Note that the reported cross sections are cumulative and include the formation contribution from the short-lived parent isomer ${ }^{73 \mathrm{~m}} \mathrm{Se}\left(t_{1 / 2}=39.8(13) \mathrm{min}\right)$ in addition to the longer-lived $\left(t_{1 / 2}=7.15(8) \mathrm{h}\right)$ ground state [81]. The results of the BNL irradiation help to extend the excitation function and characterize its tail behaviour up to $200 \mathrm{MeV}$. The consistency between our results and the literature data compiled in EXFOR builds confidence in the energy and current assignments determined in this work as well as the overall measurement and data analysis methodology.

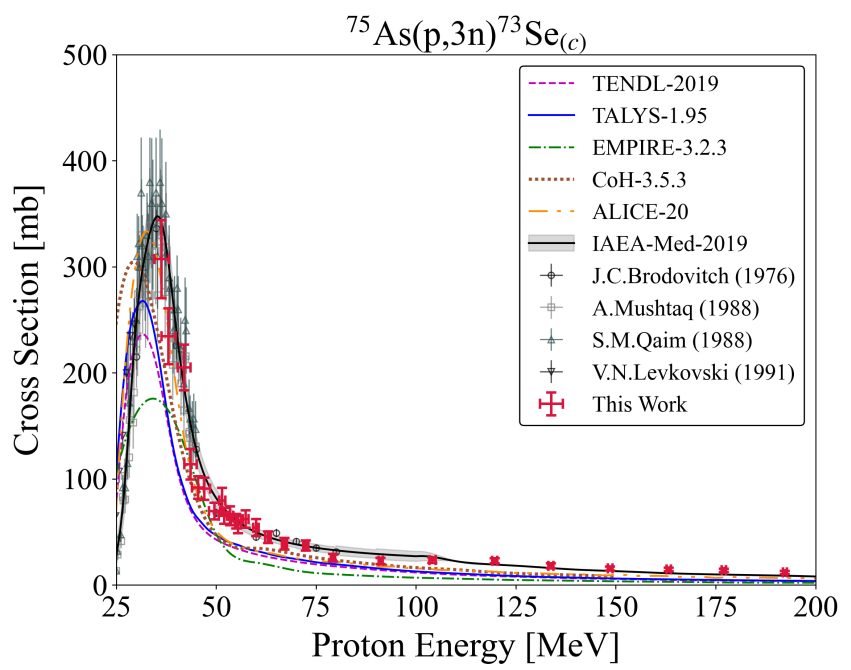

FIG. 7. Experimental and theoretical cross sections for ${ }^{73} \mathrm{Se}$ production, peaking near $330 \mathrm{mb}$ around $35 \mathrm{MeV}$.

The default TALYS and EMPIRE predictions both underestimate the compound peak magnitude, EMPIRE decidedly more so than TALYS, while TALYS also shifts the peak energy lower than experimentally observed. The ALICE calculation performs best here with an appropriate peak magnitude and nearly proper tail shape, which is just incorrectly shifted similar to TALYS. TENDL replicates TALYS very closely other than a slightly reduced peak magnitude. $\mathrm{CoH}$ significantly mispredicts the channel's rising edge resulting in a more severe energy shift than both TALYS and ALICE.

The measured falling edge of the compound peak is additionally relevant to the medical community as
${ }^{75} \mathrm{As}(\mathrm{p}, 3 \mathrm{n})$ has been shown as the most advantageous route to the nonstandard positron emitter ${ }^{73} \mathrm{Se}[82]$. In this vein, the production of ${ }^{73} \mathrm{Se}$ has also been evaluated by the IAEA and this recommended fit is given in Figure 7 [3]. The IAEA fit is seen to agree very well with the measured data in this paper.

It is worth noting that although the cross section averages only $\approx 40 \mathrm{mb}$ from $50-200 \mathrm{MeV}$, the greater range of incident protons at $200 \mathrm{MeV}$ as compared to $50 \mathrm{MeV}$ would lead to a more than doubling in the overall ${ }^{73} \mathrm{Se}$ production yield. This brief consideration is representative of the value inherent to high-current, high-energy proton accelerator facilities and rationalizes the effort to measure high-energy reaction data for potential production targets such as arsenic.

\section{D. ${ }^{75} \mathbf{A s}(\mathbf{p}, \mathbf{p 3 n}){ }^{72}$ As Cross Section}

The direct measurement of ${ }^{72}$ Se decay allowed for the subsequent independent cross section quantification of ${ }^{72}$ As. The cross section results are presented in Figure 8 and are the measured first data for this reaction channel.

The modeling predictions all perform similarly in this channel, in contrast to the large variations seen for nearby ${ }^{72}$ Se and ${ }^{73}$ Se production. EMPIRE, CoH, and ALICE underpredict the high-energy pre-equilibrium tail for ${ }^{72}$ As relative to TALYS and TENDL, though the former trio of codes have the better energy placement of the compound peak centroid.

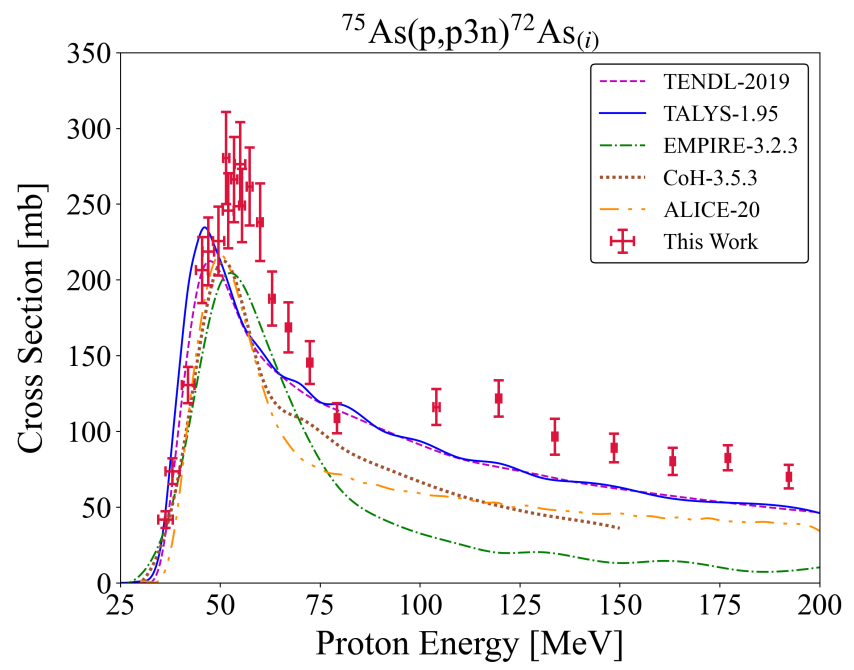

FIG. 8. Experimental and theoretical cross sections for ${ }^{72} \mathrm{As}$ production, peaking near $275 \mathrm{mb}$ around $55 \mathrm{MeV}$.

\section{E. ${ }^{\text {nat }} \mathbf{T i}(\mathbf{p}, \mathbf{x})^{44 \mathrm{~m} / \mathrm{g}} \mathbf{S c}$ Cross Section}

The production of ${ }^{44 \mathrm{~g}} \mathrm{Sc}\left(t_{1 / 2}=3.97\right.$ (4) $\mathrm{h}$ [83] $)$ is of general interest as an emerging radiometal for nuclear imaging and theranostic purposes [3, 82, 84, 85]. 
While the measurements of the ${ }^{\text {nat }} \mathrm{Ti}(\mathrm{p}, \mathrm{x})^{44 \mathrm{~m} / \mathrm{g}} \mathrm{Sc}$ excitation functions extracted from the titanium monitor foils included in the target stacks may not give an ideal production route for this medical application, these cross section results do give the only observable isomer and ground state pair from the three irradiations. As a result, this work provides a large update to the ${ }^{44 \mathrm{~m}} \mathrm{Sc}$ $\left(t_{1 / 2}=58.61(10) \mathrm{h}, J^{\pi}=6^{+}\right)$to ${ }^{44 \mathrm{~g} S c}\left(t_{1 / 2}=3.97\right.$ (4) h, $\left.J^{\pi}=2^{+}\right)$[83] isomer-to-ground state ratio via ${ }^{\text {nat }} \mathrm{Ti}(\mathrm{p}, \mathrm{x})$, as seen in Figure 9 and recorded in Table V.

TABLE V. Isomer-to-ground state production ratio for ${ }^{\text {nat }} \mathrm{Ti}(\mathrm{p}, \mathrm{x})^{44 \mathrm{~m} / \mathrm{g}} \mathrm{Sc}$ covering incident proton energies from 36 to $192 \mathrm{MeV}$.

\begin{tabular}{lc}
\hline \hline$E_{\mathrm{p}}[\mathrm{MeV}]$ & $\sigma\left({ }^{44 \mathrm{~m}} \mathrm{Sc}\right) / \sigma\left({ }^{44 \mathrm{~g}} \mathrm{Sc}\right)$ \\
\hline $192.26(49)$ & $0.456(29)$ \\
$176.99(51)$ & $0.449(32)$ \\
$163.18(54)$ & $0.455(25)$ \\
$148.52(58)$ & $0.471(21)$ \\
$133.72(62)$ & $0.508(34)$ \\
$119.63(67)$ & $0.470(37)$ \\
$104.05(74)$ & $0.603(59)$ \\
$91.05(51)$ & $0.664(45)$ \\
$79.15(57)$ & $0.566(37)$ \\
$72.34(61)$ & $0.650(48)$ \\
$62.87(67)$ & $0.598(40)$ \\
$59.88(70)$ & $0.577(40)$ \\
$57.26(72)$ & $0.668(88)$ \\
$55.36(74)$ & $0.548(25)$ \\
$54.9(13)$ & $0.563(34)$ \\
$53.40(76)$ & $0.576(26)$ \\
$51.9(14)$ & $0.472(27)$ \\
$51.39(79)$ & $0.554(25)$ \\
$49.5(14)$ & $0.595(40)$ \\
$46.9(15)$ & $0.529(37)$ \\
$45.4(15)$ & $0.521(39)$ \\
$43.5(16)$ & $0.512(39)$ \\
$31.9(16)$ & $0.488(23)$ \\
$36.2(18)$ & $0.505(23)$ \\
\hline & $0.399(15)$ \\
\hline 5$)$ &
\end{tabular}

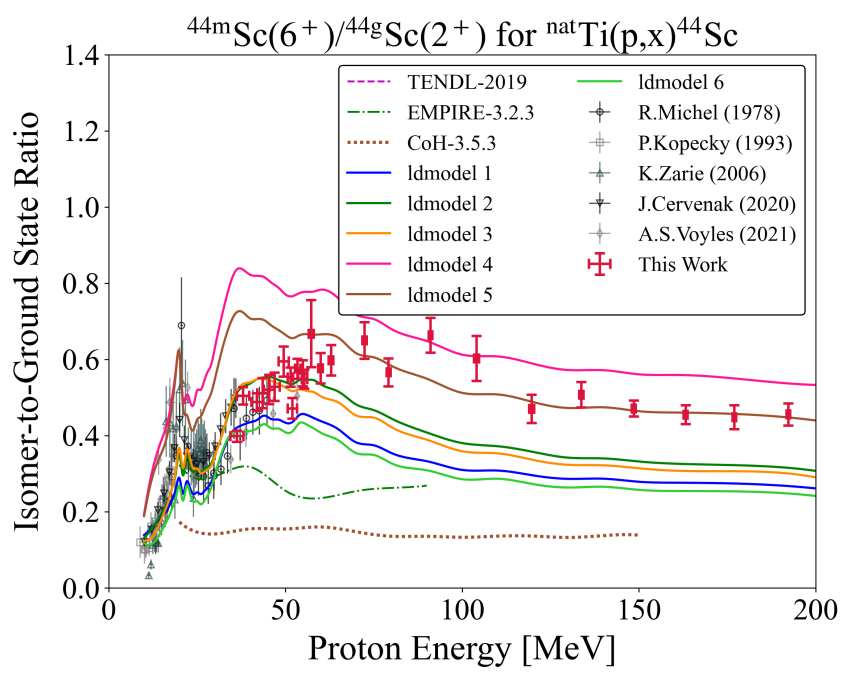

FIG. 9. Experimental and theoretical results for the isomerto-ground state production ratio for ${ }^{\text {nat }} \mathrm{Ti}(\mathrm{p}, \mathrm{x})^{44 \mathrm{~m} / \mathrm{g}} \mathrm{Sc}$. The predictions from all 6 TALYS level density models are shown, where ldmodel 1 is equivalent to the TALYS default.

Multiple experiments have measured this ratio previously for less than $50 \mathrm{MeV}$ and there is agreement between the high-energy end of those measurements and the lowest-energy results of this work [33, 49, 52-54]. This new data extension could be used by the reaction modeling community to gain insight into angular momentum deposition over a broad range of incident particle energies.

The EMPIRE, CoH, and TENDL predictions for the isomer-to-ground state ratio are also shown in Figure 9 for comparison. The EMPIRE and $\mathrm{CoH}$ predictions markedly underestimate the ratio, however this result is a function of varying errors. In EMPIRE's case, the ratio is incorrect due to an overestimation of nat $\mathrm{Ti}(\mathrm{p}, \mathrm{x})^{44 \mathrm{~g}} \mathrm{Sc}$ production (see Figure 22 in Appendix C) while the $\mathrm{CoH}$ misprediction is instead a function of underestimation for ${ }^{\text {nat }} \mathrm{Ti}(\mathrm{p}, \mathrm{x})^{44 \mathrm{~m}} \mathrm{Sc}$ production (Figure 23 in Appendix $\mathrm{C}$ ).

In the compound peak energy region of the ${ }^{44 \mathrm{~m} / \mathrm{g}} \mathrm{Sc}$ excitation functions $(25-45 \mathrm{MeV})$, competition with other exit residual product channels is minimized. Hence the optical model impact and transmission coefficient effects are minimized and the isomer-to-ground state data in Figure 9 is largely a function of the level density of ${ }^{44} \mathrm{Sc}$. Consequently, comparing the isomer-to-ground state predictions from TALYS's numerous nuclear level density models is a conventional brief investigation of this data. These TALYS predictions are the remaining comparisons shown in Figure 9.

The ldmodel 1 in TALYS is the default GilbertCameron constant temperature and Fermi gas model, but ldmodel 2, the Back-shifted Fermi gas model, appears to perform best in Figure 9 over the largest energy range. Though, it is perhaps noteworthy that the highenergy portion of the data is best reproduced by two of TALYS's microscopic level density models - Idmodel 4 and ldmodel 5. The exact nature of these microscopic models, and all six models in total, can be reviewed in 
the TALYS-1.95 manual [34].

A single iteration of the Fox et al. [21] fitting procedure was additionally applied for nat $\mathrm{Ti}(\mathrm{p}, \mathrm{x})$ to try and glean more insight on the effect of level density choice for the relevant nuclei. It was found that an overall best fit to the multiple observed residual product channels (see Table IV for product list) was still achieved using 1 dmodel 2 but that an energy-dependent increase in the spin cut-off parameter was also included among the model adjustments. The spin cut-off increase, set by the procedure to begin globally at $E_{p}=40 \mathrm{MeV}$ in this case, broadens the width of the angular momentum distribution of the level densities involved in the ${ }^{\text {nat }} \mathrm{Ti}(\mathrm{p}, \mathrm{x})$ reaction [34]. This adjusted best fit can be seen versus the unadjusted ldmodel 2 case for the isomer-to-ground state ratio in Figure 10. The mispredictions of the adjusted fit beyond $\approx 60 \mathrm{MeV}$ are not necessarily unexpected since these calculations are significantly complicated due to a polyisotopic target. However, since special attention was paid to adjusting this ${ }^{44 \mathrm{~m} / \mathrm{g}}$ Sc ratio, the lasting mispredictions are likely more attributable to fundamental issues in the base pre-equilibrium model rather than parameter tuning.

It is interesting to observe that beyond $\approx 125 \mathrm{MeV}$, the ratio remains relatively constant, thereby indicating a limit to the maximum amount of angular momentum that can be imparted to the system. This is a reflection of the mechanics of the pre-equilibrium process.

This is evidently only an elementary investigation of the angular momentum in ${ }^{44} \mathrm{Sc}$ and neighbouring nuclei, and a detailed investigation is outside the intent of this paper. Altogether, this discussion is still presented to inform the value and scarcity of these types of ratio datasets over wide energy regions, and to provide motivation for further analysis.

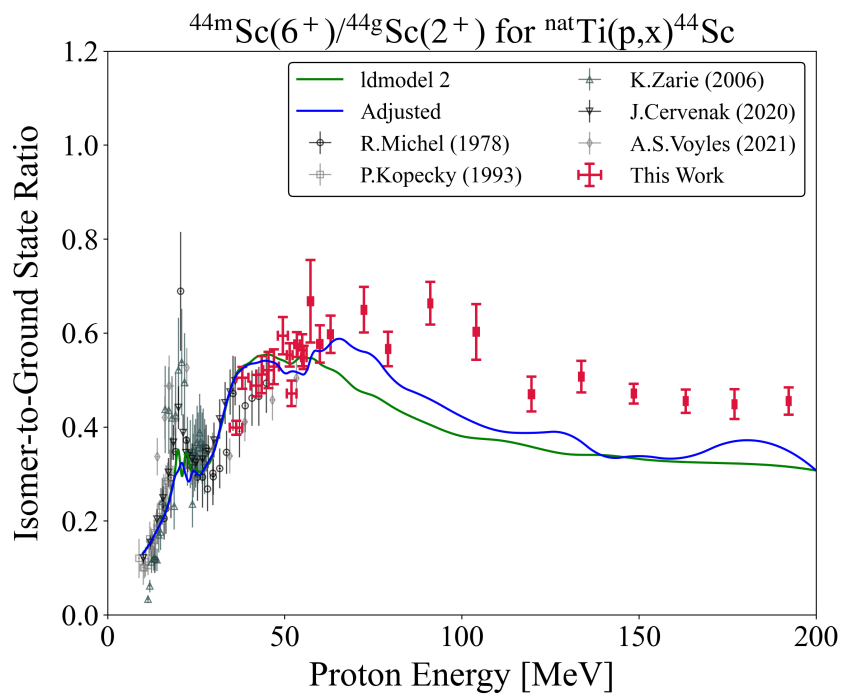

FIG. 10. Comparison of the TALYS ldmodel 2 model prediction for the isomer-to-ground state production ratio for ${ }^{\text {nat }} \mathrm{Ti}(\mathrm{p}, \mathrm{x})^{44 \mathrm{~m} / \mathrm{g}} \mathrm{Sc}$ with a TALYS fit using adjusted parameters, including a spin cut-off increase.

\section{F. ${ }^{\text {nat }} \mathbf{C u}(\mathbf{p}, \mathbf{x})$ Cross Sections}

The numerous ${ }^{\text {nat }} \mathrm{Cu}(\mathrm{p}, \mathrm{x})$ cross sections measured here are in good agreement with the existing body of literature data and help to populate the more sparse regions of measurements between 100-200 MeV. Plots of these copper excitation functions are provided in Appendix C. Similar to the ${ }^{73}$ Se results (Figure 7 ), the ${ }^{\text {nat }} \mathrm{Cu}(\mathrm{p}, \mathrm{x})$ comparisons with existing data lend credence to our analysis methodology as well as our extensions to energy regions with no prior cross section measurements.

\section{G. Predicted Physical Thick Target Yields}

Instantaneous thick target yields for ${ }^{75} \mathrm{As}(\mathrm{p}, \mathrm{x}){ }^{72} \mathrm{Se},{ }^{68} \mathrm{Ge}$ were calculated from the measured cross section results and are plotted in Figure 11. A comparison to the yields from earlier discussed established production routes for these generator nuclei in Section I are also included.

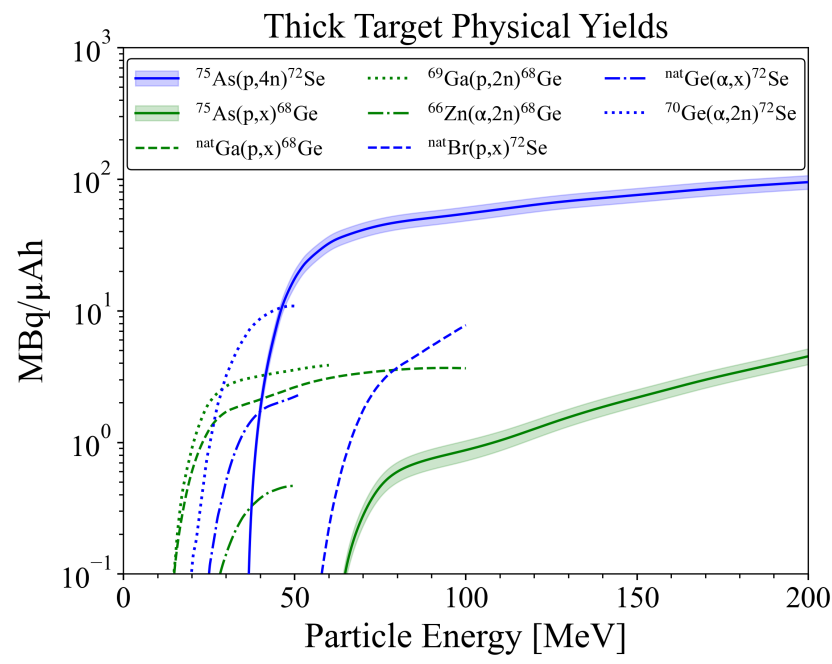

FIG. 11. Yields for the PET generator radionuclides ${ }^{72}$ Se and ${ }^{68} \mathrm{Ge}$ according to established production routes and the new arsenic-based routes measured in this work [13, 14, 42, 86-93].

The data from TREND suggests that across all relevant incident particle energies beyond reaction threshold, the ${ }^{75} \mathrm{As}(\mathrm{p}, 4 \mathrm{n}){ }^{72} \mathrm{Se}$ is the optimal production pathway to the ${ }^{72} \mathrm{Se} /{ }^{72} \mathrm{As}$ generator system. The arsenic target route offers an increase in yield of greater than an order of magnitude versus the current methods, while still affording radioisotopically pure production as best as possible. Specifically, no charged-particle production route to the ${ }^{72} \mathrm{Se} /{ }^{72} \mathrm{As}$ generator system is uncontaminated from ${ }^{75-73} \mathrm{Se}$ co-production. However, it is expected that ${ }^{72} \mathrm{As}$ will be efficiently separated from the parent ${ }^{72} \mathrm{Se}$ when needed, and that the co-produced ${ }^{75-73}$ Se will also follow the chemical separation $[9,11]$. Of course, any ${ }^{73} \mathrm{Se}$ contaminant is much shorter lived than ${ }^{72}$ Se and can be decayed out to reach a more pure ${ }^{72}$ Se starting condition 
regardless. Further, ${ }^{75} \mathrm{Se}$ production is energetically unfavorable in the $\mathrm{p}+{ }^{75} \mathrm{As}$ production conditions for ${ }^{72} \mathrm{Se}$, meaning any in-grown ${ }^{75} \mathrm{As}$ prior to separation will be both minimal and stable. The ${ }^{75} \mathrm{As}(\mathrm{p}, 4 \mathrm{n})$ pathway also avoids any potential long-lived ${ }^{74,73,71}$ As contamination present from Ge target routes. In total, arsenic-based production of ${ }^{72} \mathrm{Se}$ gives the best chance to produce and collect a radiochemically-pure ${ }^{72} \mathrm{As}$ daughter.

It is seen that at incident proton energies nearing $200 \mathrm{MeV}$, the yield from ${ }^{75} \mathrm{As}(\mathrm{p}, \mathrm{x}){ }^{68} \mathrm{Ge}$ can rival and exceed the production route based on already employed natural gallium targets. Specifically, Figure 11 predicts an $\approx 18 \%$ increase for the arsenic-based yield at $200 \mathrm{MeV}$ $(4.5>3.8 \mathrm{MBq} / \mu \mathrm{Ah})$. Nevertheless, a $\mathrm{p}+{ }^{75} \mathrm{As}$ approach is expected to co-produce more stable germanium and ${ }^{71} \mathrm{Ge} \rightarrow{ }^{71} \mathrm{Ga}$ contamination versus the $\mathrm{p}+{ }^{\text {nat }} \mathrm{Ga}$ route, leading to reduced ${ }^{68} \mathrm{Ge}$ specific activity. Arsenic targets would also introduce a need for additional, potential lossy, separation chemistries due to long-lived selenium and arsenic products not present from $\mathrm{p}+{ }^{\text {nat }} \mathrm{Ga}$. Therefore, uprooting the successful established gallium route for arsenic is unwarranted. Still, this ${ }^{75} \mathrm{As}(\mathrm{p}, \mathrm{x})^{68} \mathrm{Ge}$ study gives valuable information in the context of total arsenic reactions, contributes to the knowledge base of the essential ${ }^{68} \mathrm{Ge} /{ }^{68} \mathrm{Ga}$ system, and demonstrates the importance of measuring these high-energy reactions, which can very easily produce large yields due to the long range of high-energy protons.

\section{CHARGED-PARTICLE REACTION MODELING}

The effort to explore and improve the current nuclear reaction models for charged-particles, and perhaps more specifically charged-particles at high incident energies, is continued in this work. Explicitly, the TALYS residual product based fitting procedure presented by Fox et al. [21] is applied to ${ }^{75} \mathrm{As}(\mathrm{p}, \mathrm{x})$ given the unique, large body of proton-induced data measured here.

The nine reaction channels ${ }^{75} \mathrm{As}(\mathrm{p}, \mathrm{x})^{75,73,72} \mathrm{Se}$, ${ }^{74,73,71} \mathrm{As},{ }^{69} \mathrm{Ge},{ }^{68,67} \mathrm{Ga}$ were simultaneously used for the parameter adjustment investigation. ${ }^{73} \mathrm{Se},{ }^{73} \mathrm{As}$, ${ }^{69} \mathrm{Ge}$, and ${ }^{68} \mathrm{Ga}$ were considered as the most important fitting cases due to a combination of factors such as cross section magnitude, diversification of particle emission types, and impact on production competition with neighbouring nuclei.

\section{A. Deformation Effect of ${ }^{75}$ As}

While the cases of ${ }^{93} \mathrm{Nb}(\mathrm{p}, \mathrm{x})$ in Fox et al. [21] and of ${ }^{75} \mathrm{As}(\mathrm{p}, \mathrm{x})$ here have similar attributes - both utilize data from the same experiments, which cover the same energy range of interest, and both are monoisotopic targets in nearby mass ranges - the documented deformation of ${ }^{75} \mathrm{As}$ is a notable change from the spherical ${ }^{93} \mathrm{Nb}$
[94-97]. This potentially introduces a complication to the direct application of the fitting procedure from Fox et al. [21]. Specifically, it would be necessary to address coupled-channels (CC) calculations or other angular momentum modifications to the typical spherically symmetric Hauser-Feshbach formalism prior to any further parameter changes [98].

The RIPL-3 imported TALYS value for the ${ }^{75} \mathrm{As}$ quadrupole deformation parameter is -0.25 , which suggests a strongly oblate deformation [34, 99]. In fact, RIPL-3 lists strong oblate deformation for the arsenic isotopes $A=68-76$. While some experimental evidence supports these values for the neutron deficient cases and transitions around $N=Z$, it is quite rare that the neutron rich isotopes would demonstrate oblate rather than prolate deformation [100, 101]. An investigation using a Nilsson diagram gives further support that ${ }^{75} \mathrm{As}$ is actually prolate in nature. Finally, ENSDF and the original datasets incorporated into the structure evaluation provide experimental evidence of the prolate condition for ${ }^{75} \mathrm{As}$ and actually list a quadrupole deformation parameter of +0.314 (6) [94]. This prolate value appears to be both physically and historically more correct than the RIPL-3 $\beta_{2}=-0.25$ and is therefore taken as the ${ }^{75} \mathrm{As}$ deformation in the analysis that follows.

TALYS, however, does not include any deformation coupling schemes for arsenic isotopes and as a result, a spherical OMP basis is used in the predictive calculations, thereby potentially neglecting a significant physics aspect of the problem. It was therefore necessary to manually create a coupling scheme to see whether this has an effect on final results. Yet, the level scheme of ${ }^{75}$ As does not present any ideal vibrational or rotational bands for coupling and its deformation is very likely either soft vibrational or soft rotational [102, 103].

On further examination, the $3 / 2^{-}$ground state with the $5 / 2^{-}$level at $279.543 \mathrm{keV}$ and the $7 / 2^{-}$level at $821.620 \mathrm{keV}$ appear to form a rotational band. The $5 / 2^{-}$level shows the expected strong $\gamma$-ray transition $\left(I_{\gamma}=100.0(5) \%\right)$ of M1 character to the ground state, while the $7 / 2^{-}$excited level shows both a strong E2 transition to the ground state $\left(I_{\gamma}=100.0(15) \%\right)$ and weaker M1 transition to the $5 / 2^{-}$level $\left(I_{\gamma}=9.6(11) \%\right)$, generally in line with behaviour expected from a rotational band. Further, the $7 / 2^{-}$E2 transition is 23.0 (24) Weisskopf units [104], providing evidence for its collective behaviour. This three-level rotational band coupling scheme was added to TALYS.

It was also noticed that the neighbouring nuclei ${ }^{76,74} \mathrm{Se}$ and ${ }^{76,74} \mathrm{Ge}$ demonstrate vibrational character $[105,106]$ and have vibrational coupling schemes implemented in TALYS for CC calculations $\left({ }^{76} \mathrm{Ge}\right.$ has actually recently been shown as rigid triaxially deformed [107]). These neighbouring properties provide motivation to model the arsenic target as soft vibrational rather than rotational.

Unfortunately, TALYS's implementation of the ECIS06 code for optical model and CC calculations is unsuited for a pure vibrational coupling scheme for odd-Z 
nuclei, and the weak-coupling model has to be used in such cases. Moreover, the only odd-Z nucleus with any sort of vibrational deformation file in TALYS is ${ }^{241} \mathrm{Am}$, where vibrational collectivity is built on top of rotational character. Therefore, taking the ${ }^{241}$ Am deformation formatting as a guide, a weak vibrational band consisting of the ${ }^{75} \mathrm{As} 9 / 2^{+}(303.9243 \mathrm{keV}), 5 / 2^{+}(400.6583 \mathrm{keV})$, and $1 / 2^{+}(860.0 \mathrm{keV})$ levels were added to a second created coupling scheme including the prior discussed rotational band. In this suggested vibrational band, the $1 / 2^{+}$level is dominated by transition to $5 / 2^{+}$, which then has an E2 transition to the $9 / 2^{+}$of 76.4 (25) Weisskopf units [104]. The $9 / 2^{+}$de-excitation is dominated by E3 decay to the ground state. This mixed rotational+vibrational coupling scheme was also added to TALYS.

Elsewhere, this treatment for adjusting the global spherical optical model by a CC approach to implement a deformed optical model for ${ }^{75}$ As calculations has been used in Shibata et al. [103] and Kawano [102]. The Shibata et al. [103] work is an evaluation of neutron nuclear data on ${ }^{75} \mathrm{As}$ up to $20 \mathrm{MeV}$ for JENDL-4 and uses a similar rotational coupling scheme to the one presented here but substitutes the $5 / 2^{-}$level at $279.543 \mathrm{keV}$ with a $5 / 2^{-}$level at $572.41 \mathrm{keV}$. Shibata et al. [103] uses the quadrupole deformation parameter $\beta_{2}=-0.19$ within a rigid-rotator model. In their evaluation, they found it necessary to additionally tune the matrix element parameter as well as the pickup and knockout contributions for their pre-equilibrium model relevant to the residual product cross sections of $(n, \gamma),(n, p),(n, 2 n)$, and $(n, \alpha)$. However, the JENDL-4 evaluation still found limited success in fitting the ${ }^{75} \mathrm{As}(\mathrm{n}, \mathrm{p})$ channel after accounting for both deformation and pre-equilibrium changes. Shibata et al. [103] considered other solutions attempts that included level density and optical model parameter changes concerning both ${ }^{75} \mathrm{As}$ and ${ }^{75} \mathrm{Ge}$ but could not simultaneously improve the (n,p) channel while maintaining good global behaviour elsewhere.

Kawano [102] performed their CC calculations using the $\mathrm{CoH}$ reaction code and probed the collectivity effects in ${ }^{75} \mathrm{As}$ for incident neutrons. They explored the total and some close-to-target residual product cross sections up to $20 \mathrm{MeV}$, similar to Shibata et al. [103]. In comparison to ENDF/B-VII.0 results, the Kawano [102] calculations demonstrated improvement in reproducing the total cross section but did require model parameter adjustments for the individual reaction channels, not always yielding satisfactory results. Kawano [102] used the RIPL-3 suggested strong oblate deformation of arsenic.

In this $\mathrm{p}+{ }^{75} \mathrm{As}$ modeling work, the $\mathrm{CC}$ calculations in TALYS for arsenic, when invoking either the custom rotational+vibrational deformation or the custom pure rotational deformation scheme, together with the ENSDFaccepted prolate deformation parameter $\beta_{2}=+0.314$ (6), proved to have minimal impact on the predictions for residual product excitation functions. Any alterations that were present were not seen to be consistent improvements versus the default spherical optical model calcula- tions. This is not an entirely unusual result given the higher energies under consideration and the overall expected lower level of collectivity for this target nucleus. It should be noted that this is not an exhaustive investigation of arsenic deformation, CC calculations, or collectivity models, and no structure or theory statements can be made. This result is only a statement of the sensitivity of the modeling under the conditions of this work.

Given the observed unremarkable changes, the inability to disentangle effects of $\mathrm{CC}$ calculations from more dominating level density, optical model, and preequilibrium parameter adjustments, and the imperfections of previously established deformed fitting approaches, the decision was made to treat ${ }^{75}$ As spherically within TALYS and implement the fitting procedure from Fox et al. [21] identically.

\section{B. Fitting Procedure Applied to ${ }^{75} \mathrm{As}(\mathbf{p}, \mathbf{x})$}

Firstly, the application of microscopic level density models proved beneficial as compared to the default phenomenological Gilbert-Cameron constant temperature model or the placement of compound peak centroids. However, it was seen that no one microscopic level density model best reproduced the excitation functions across all the observables. Instead, level density calculations from Goriely's tables using the Skyrme effective interaction (1dmodel 4) [108] proved to be most accurate for the close-to-target residual products, and specifically for ${ }^{72-76}$ Se and their competition with closeto-target arsenic products. Yet, applying ldmodel 4 to all nuclei involved in ${ }^{75} \mathrm{As}(\mathrm{p}, \mathrm{x})$ created pre-equilibrium tails biased too high above the experimental data for $\mathrm{Ga}, \mathrm{Ge}$, and other $\alpha$-emission residual product excitation functions farther from the target. Conversely, it was observed that the temperature-dependent Hartree-FockBogolyubov level density calculations using the Gogny force (1dmodel 6) [109] did not suffer from the magnitude bias problems in the far-from-target channels, but failed to model the close-to-target Se and their competing products unlike Idmodel 4 . Therefore, two microscopic level density models were used, where ldmodel 4 was applied to the aforementioned grouping of selenium nuclei and ldmodel 6 was applied for all else. Further details of these level density considerations can be reviewed in Section IV B 1.

The pre-equilibrium parameter adjustments in the next portion of the procedure were indeed found to follow the systematic trend described in Fox et al. [21], with M2constant $=0.80$, M2limit $=3.9$, and M2shift $=0.55$. Furthermore, the value for the constant of the proton and neutron single-particle level density parameter used for calculations of the exciton model particle-hole state densities was altered from its default $\mathrm{Kph}=15$ to $\mathrm{Kph}=15.16$. Other pre-equilibrium modeling changes were manipulations of the stripping and knockout reaction contributions for outgoing alpha, deuteron, triton, and ${ }^{3} \mathrm{He}$ par- 
ticles. These manipulations were performed using the TALYS Cstrip and Cknock keywords. The precise adjusted values can be viewed in Table IX in Appendix D.

Subsequent iterative simultaneous tuning of optical model and individual level density parameters were needed to aid the compound reaction regime and to fix erroneous production competitions between clustered products.

The need for nuclide-specific level density changes arises from discrepancies between measured and modeled data where global changes to exciton or optical model parameters can not resolve the singular problems. These nuclide-specific adjustments were most evident for ${ }^{73} \mathrm{As}$ production, where both the adjusted fit to this point and the default calculation were nearly $200 \mathrm{mb}$ smaller than the observed results. As in Fox et al. [21], these level density manipulations per nuclide could be performed with the TALYS ctable and ptable commands when microscopic level density models are implemented.

The effects of ctable and ptable to create an adjusted level density $\rho\left(E_{x}, J, \pi\right)$ are explicitly given by,

$$
\rho\left(E_{x}, J, \pi\right)=\exp \left(c \sqrt{E_{x}-\delta}\right) \rho_{m i c}\left(E_{x}-\delta, J, \pi\right),
$$

where ctable is the $c$ constant, ptable is the $\delta$ constant (denoted as the "pairing shift"), and $\rho_{\text {mic }}\left(E_{x}-\delta, J, \pi\right)$ are the tabulated microscopic level density calculations as a function of excitation energy $E_{x}$, angular momentum $J$, and parity $\pi$. The produced tables in TALYS have not been adjusted to experimental data and have $c=0$ and $\delta=0$ by default. The implementation of ctable and ptable under the definition of Equation (2) then provides necessary scaling flexibility at both low and high energies [34].

Since the production of ${ }^{73} \mathrm{As}$ is most heavily correlated with the neighbouring exit channels ${ }^{72,73} \mathrm{Se}$ and ${ }^{74} \mathrm{As}$, the ctable and ptable effects on ${ }^{73}$ As necessitated corresponding nuclide-specific level density changes in ${ }^{72,73} \mathrm{Se}$ and ${ }^{74} \mathrm{As}$ as well.

The most suitable optical model adjustments were found to be d1adjust $\mathrm{n}=1.75$ and d1adjust $\mathrm{p}=1.55$, which multiply the energy-dependent imaginary surfacecentral potential well depth for neutron and protons, respectively. These multiplicative changes lead to increased particle emission from the surface region of the nucleus, and thus to increased emission of high-energetic particles, particularly at lower incident proton energies. In turn, these alterations create a more pronounced preequilibrium spectrum that contributes additional production within the compound regions of residual product excitation functions and some additional production to their tails.

Although these are not unsubstantial multiplication factors from the default 1.0 values, the energy dependence of the surface potential means that the adjustment impact is large in the vicinity of low threshold channels for lower incident proton energies but becomes only a minor change above $\approx 50 \mathrm{MeV}$. This is mirrored by the volume potentials that increase and dominate absorption/emission as energies reach $\approx 50 \mathrm{MeV}$ and beyond. For example, at $E_{p}=20 \mathrm{MeV}$, the default imaginary surface-central potential well depth for protons on ${ }^{75} \mathrm{As}$ is $8.4 \mathrm{MeV}$ while the adjusted well depth is $1.55 \times$ larger at $13.0 \mathrm{MeV}$. This $4.6 \mathrm{MeV}$ difference is a relevant change around low residual product threshold energies but by $E_{p}=75 \mathrm{MeV}$, this default versus adjusted well depth difference is reduced to just $1.5 \mathrm{MeV}$. The difference then falls below $1 \mathrm{MeV}$ at $E_{p}=90 \mathrm{MeV}$, and is reduced down to $0.1 \mathrm{MeV}$ at $E_{p}=200 \mathrm{MeV}$. Similar behaviour is true for the change to the imaginary surface-central potential well depth for neutrons. Furthermore, at $E_{p}=20 \mathrm{MeV}$, the imaginary volume potential is $5-7 \times$ smaller than the imaginary surface potential for both neutrons and protons in the adjusted case, but by $E_{p}=75 \mathrm{MeV}$, the imaginary volume potential has grown to be $2-3 \times$ larger. The imaginary volume potential becomes increasingly more dominant, growing to be $50-70 \times$ larger by $E_{p}=200 \mathrm{MeV}$.

It is possible that portions of the d1adjust changes should actually be substituted with changes to the imaginary surface diffusivity parameter, but this cannot be unambiguously determined using only residual product cross section data and instead requires angle-differential cross section information [110]. This limited diversity of high- $E_{p}$ fit data is a common theme that permeates the limitations of this approach to parameter adjustments as well as prevents much physical meaning to be gleaned from the modeling. These limitations are further explored in Section IV D.

An additional increase to proton absorptivity and emissivity across a wider range of energies, to increase peaks and tails for numerous channels consistently, was still warranted by the experimental data. This was implemented with an increase to the imaginary volume potential well depth for protons by w1adjust $\mathrm{p}=1.21$.

The default TALYS alpha optical model of Avrigeanu et al. [111] was deliberately chosen as it performed best for the considered As and Ge channels. The deuteron optical model of Han et al. [112] was applied instead of the default model from standard Watanabe folding [113]. This deuteron adjustment is minor $(\leq 5 \mathrm{mb})$ compared to the alpha model effect but does better match the experimental peak and tail behaviour in observed residual product channels for $A \leq 72$.

Lastly, an additional minor nuclide-specific case for level density adjustments that became relevant as a result of iterating over the above parameter changes was ${ }^{71} \mathrm{As}$. This adjustment included corresponding small level density changes to ${ }^{68} \mathrm{As},{ }^{69} \mathrm{Ge}$, and ${ }^{69} \mathrm{Ga}$ as a function of correlated production competition.

The lone prominent outstanding modeling discrepancy among the considered channels was an overprediction of ${ }^{67} \mathrm{Ga}$ production. It is likely that this difference represents a sensitivity limit for this fitting procedure through a manual approach. Moreover, given the massive parameter space for adjustments in TALYS, it is realistic that 
the fitting here ends in a local variance minimum, unable to perfectly match all prioritized $(\approx 15 \%$ of total cross section) and minor ( $\leq 5 \%$ of total cross section) residual products. We can correct for this ${ }^{67} \mathrm{Ga}$ error by reducing the nuclide-specific level density, but this change is likely a compensating correction in this context and does not contribute to any increase in predictive power.

All parameter changes creating this total adjusted fit are given in Table IX in Appendix D. Figure 12 presents the adjusted fit compared to the default TALYS calculation for the nine considered reaction channels up to an incident proton energy of $200 \mathrm{MeV}$.

Overall, we put forth a large number of level density scalings, either directly or as a correlation consequence, and though this is not unexpected given the prior lack of data and ambiguity for the reactions and energies of interest $[109,114]$, it is important to reflect on the intricacies of performing such a number of scalings. This discussion is presented in Section IV B 1.

Additionally, context for our suggested parameter adjustments can be gleaned from the "best" parameters file for $\mathrm{n}+{ }^{75}$ As included with TALYS-1.95 [34]. This "best" parameterization contains multiple level density scalings (with the back-shifted Fermi gas model as a base) in addition to multiple optical model real potential radii and diffusivity adjustments, some of which reach upwards of $11 \%$ changed from default and are made energydependent. Similar stripping and knock-out contributions to our suggested adjustments in this work exist as well in the "best" file. Overall, our adjustments generally work to avoid potential unphysical changes to geometry parameters and the real potential instead to focus on the imaginary potential. This focus is likely more appropriate for high-energy residual product cross section datasets versus the lower-energy scattering and resonance data important to the development of the "best" $n+{ }^{75} \mathrm{As}$ parameters.

\section{Level Density Adjustments}

Figure 13 directly shows the magnitude of all manually adjusted level density cases with reference to the base ldmodel choice. The total level density of ${ }^{73}$ As has been significantly increased (Figure 13c), as warranted by the experimental data, while a significant decrease is seen in ${ }^{67} \mathrm{Ga}$ although for less direct reasons (Figure 13i).

The recommendation of these many level density changes is substantiated by the global fit success seen in Figure 12 and described in Section IV C but still requires more scrutiny. Furthermore, it is necessary to, at minimum, consider the impact of these level density changes on the residual product channels for which there were no experimental data and were unaccounted for either independently or cumulatively throughout the fitting procedure. Specifically, due to limitations of the activation technique to measure stable or some very-short lived nuclei production, the ${ }^{75} \mathrm{As}(\mathrm{p}, \mathrm{x})^{74} \mathrm{Se},{ }^{74-70} \mathrm{Ge}$, and
73,71-69 Ga channels, for $A>65$ and $Z>30$, were hidden from the fitting observations. Accordingly, it is essential to have a "performance check" for these hidden channels, where the TALYS default and adjusted fits can be compared to monitor for any egregious shape or magnitude changes brought on by the level density adjustments.

The fit performance for stable ${ }^{74} \mathrm{Se}$ is of particular interest since had there been experimental data, the channel would have been one of the prominent excitation functions for the fitting procedure. This ${ }^{74}$ Se performance check is given in Figure 14 and the difference between the default and adjusted is certainly acceptable.

In the unobserved Ge and Ga channels, there continues to be no obviously incorrect changes from the default to adjusted cases. Magnitude differences for most of these products reach $\approx 5-7 \mathrm{mb}$ and excitation function shape continuity is maintained within expectations. The adjusted ${ }^{70} \mathrm{Ge}$ production is the most significantly changed hidden channel from default, with a maximum difference of $\approx 40 \mathrm{mb}$ in the compound peak region. Therefore, when confined to residual product datasets, there are no obvious indications that the bulk of the level density adjustments made here are not viable. Even if new experimental data were to be collected for these "hidden" channels, which disagreed with the adjusted fit, it is likely that since no drastic changes have been made, the parameters can be properly updated to include the new information.

It is also worth remarking that using multiple level density models in this work is not a qualification or statement that one model more accurately reflects physical behaviour. Instead, we can only conclude that multiple level density models, and nuclide-specific changes, were simply scalings needed to best match the available experimental data, which has been seen in other work as well $[114,115]$. There is likely no clear physical insight about the models that can be taken from these fits alone.

Perhaps some of the need for scaling is due to inconsistent or lacking discrete level data that feeds into the level density models. The residual products of interest generally exist off the line of stability and resonance parameters are unknown [115-117]. In ${ }^{70} \mathrm{As}$ and ${ }^{72} \mathrm{As}$, only 68 and 65 experimental discrete levels, respectively, as stored in the RIPL-3 database, inform the level density calculations [34]. This is compared to isotopes such as ${ }^{71,73,75} \mathrm{As}$ where over 120 experimental levels each are used.

A similar pattern exists for ${ }^{72}$ Se where only 52 experimental discrete levels inform calculations, as compared to much more well-characterized ${ }^{73-76} \mathrm{Se}$ isotopes. If there are missing levels relatively low in the level scheme, then the level density model may be adjusted to the wrong number of assumed complete levels. This lack of structure data exists for ${ }^{66,67} \mathrm{Ge}$ and ${ }^{66,68} \mathrm{Ga}$ within their respective isotope chains as well. Ultimately, it is conceivable that incomplete structure data leads to numerous compensating level density effects in this mass region, which may themselves be a key contributor to the ad- 


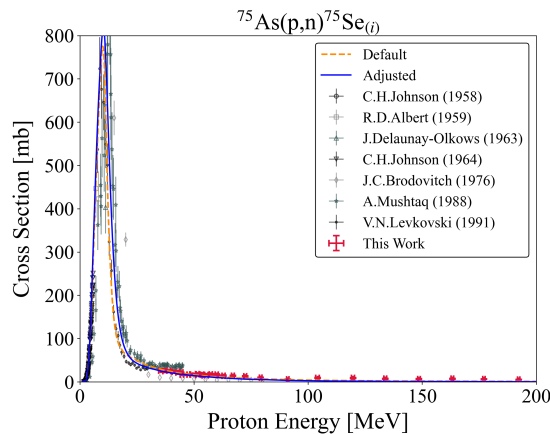

(a)

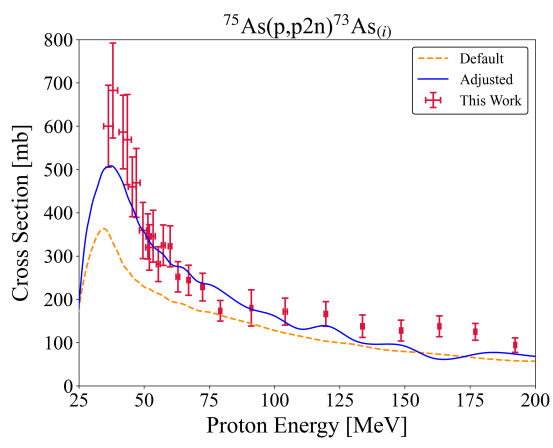

(d)

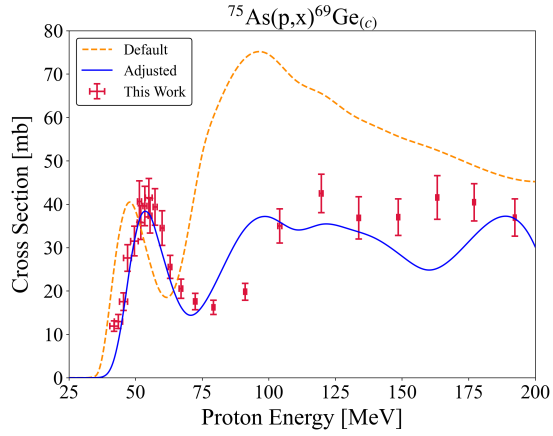

(g)

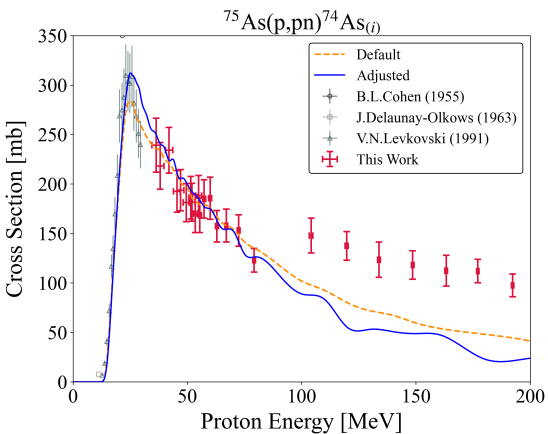

(b)

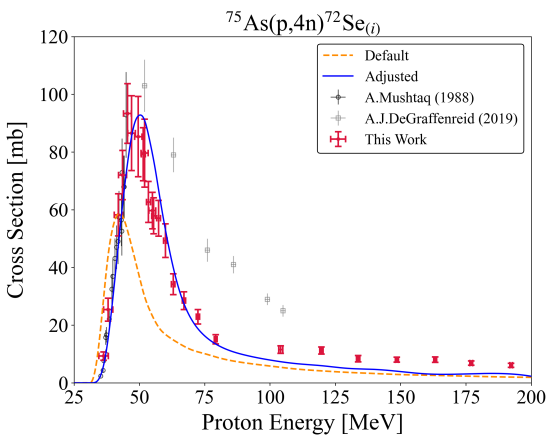

(e)

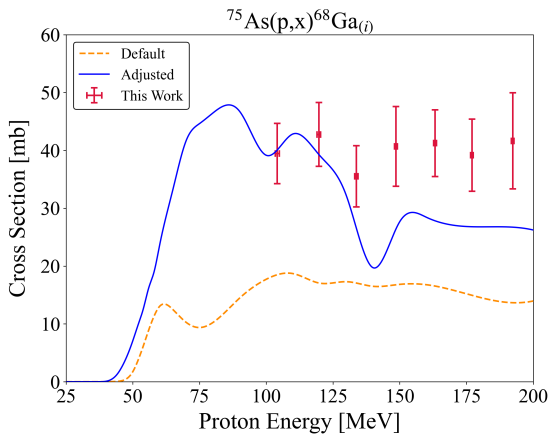

(h)

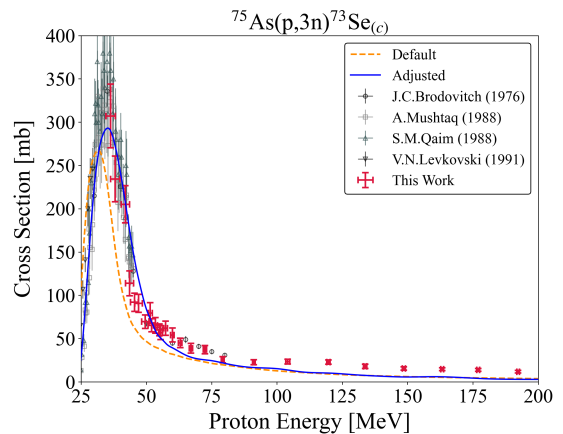

(c)

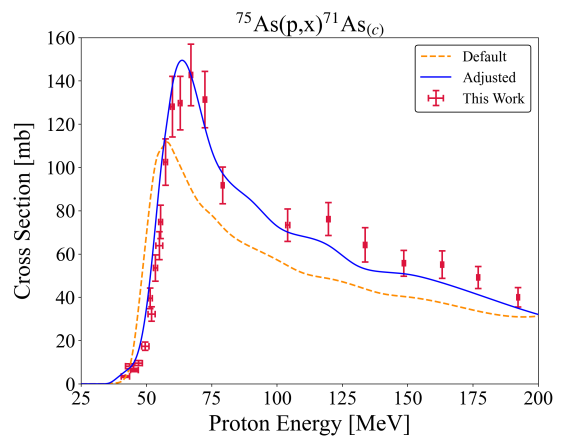

(f)

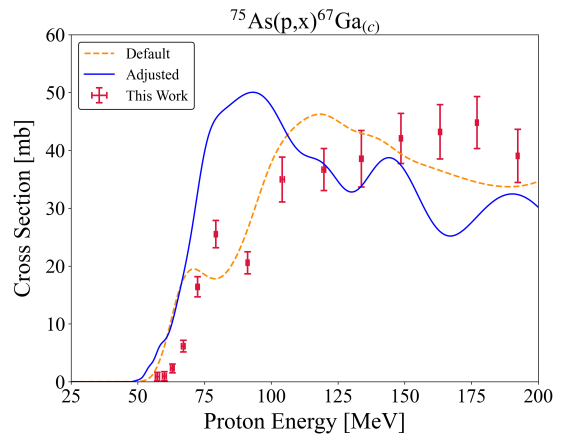

(i)

FIG. 12. TALYS default and adjusted calculations for residual products of proton-induced reactions on arsenic up to $200 \mathrm{MeV}$.

justed scalings as opposed to any inherent issues with the models [115-117].

It is also possible that a disregard of isospin effects in the current TALYS calculations, missing collective enhancement effects for nuclei far from stability, or deterioration of the microscopic level density models altogether at the high excitation energies relevant to this work, have prompted the need for the corrective scalings [117, 118]. A future experiment examining $\alpha+{ }^{72} \mathrm{Ge}$, which populates the same ${ }^{76} \mathrm{Se}$ compound system as $\mathrm{p}+{ }^{75} \mathrm{As}$ but with different isospin, could provide some additional information. Relevant future research may also include examinations of smooth transitions between different level density models as a function of mass difference from the target nucleus or separate structure-based level density model-mixing schemes. The implementation of different level density models in this manner merits specific interest because it has shown to be needed for $\mathrm{p}+{ }^{75} \mathrm{As}$ here and $\mathrm{p}+{ }^{93} \mathrm{Nb}$ in Fox et al. [21].

The overall viability of the level density adjustments in this modeling work in combination with the other modeling parameter changes are further justified in Section IV C. 


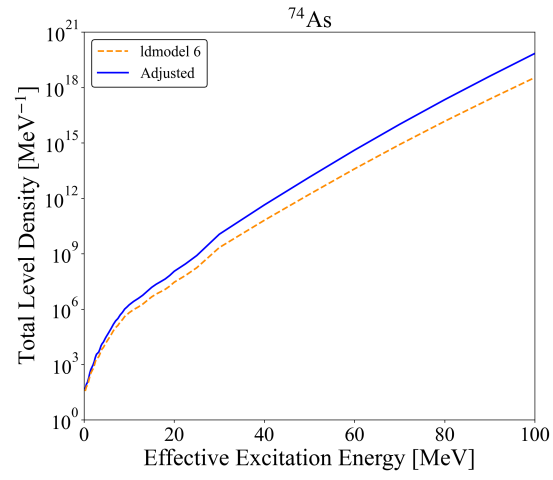

(a)

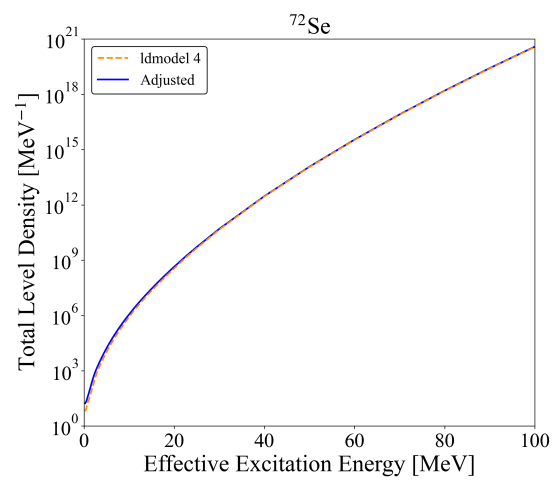

(d)

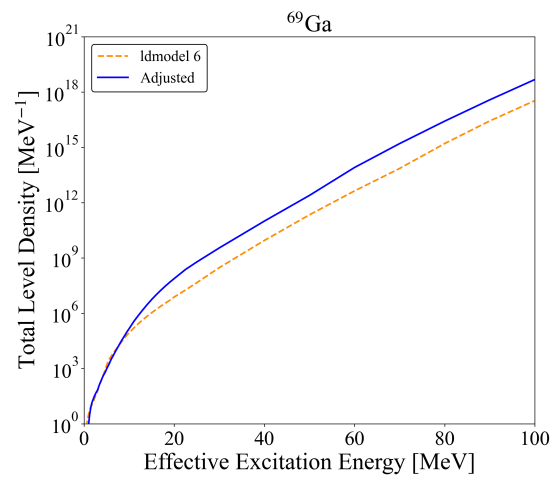

(g)

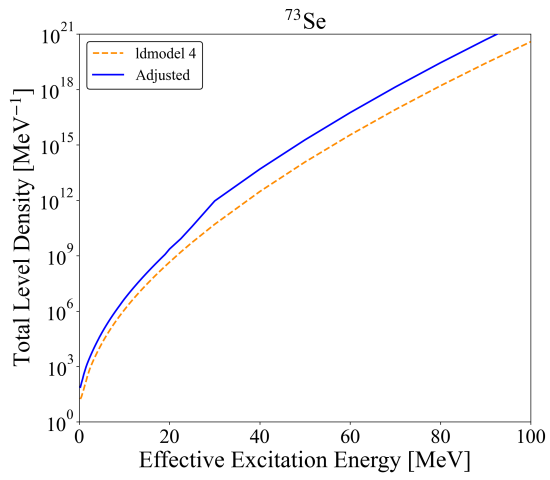

(b)

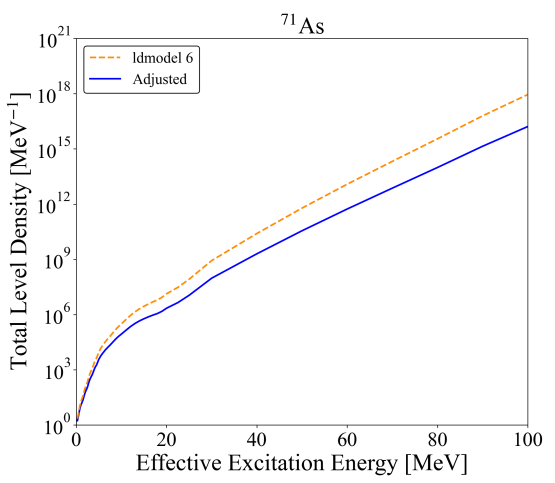

(e)

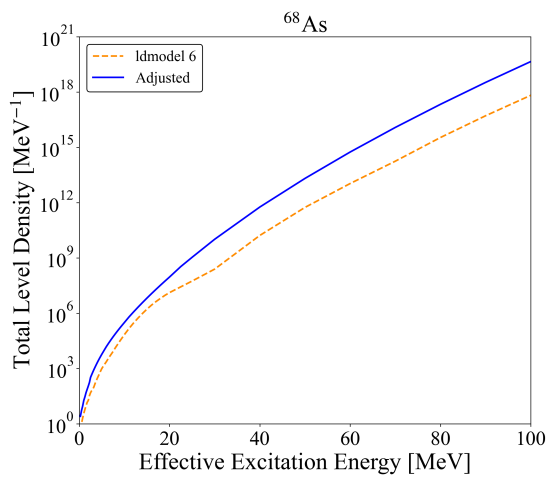

(h)

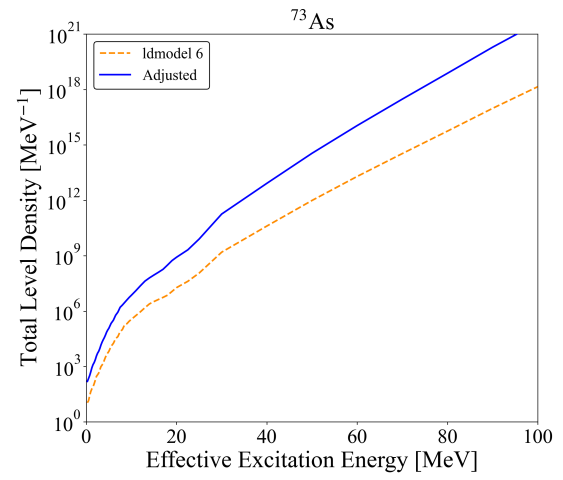

(c)

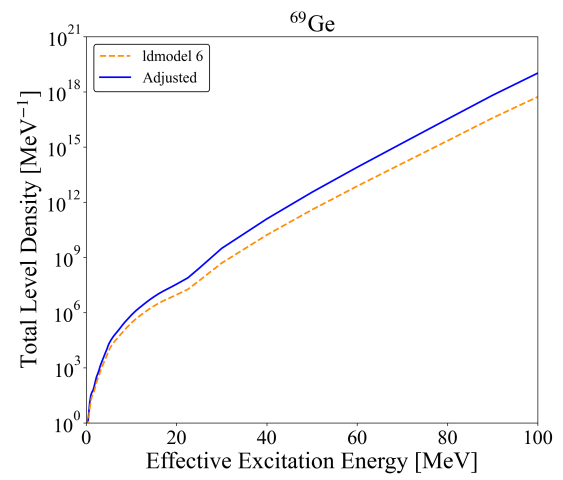

(f)

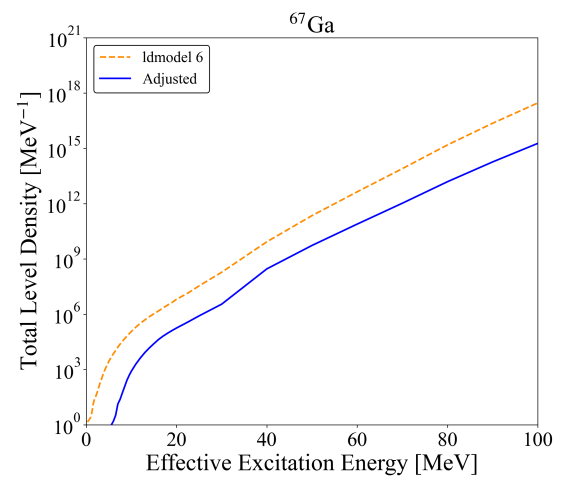

(i)

FIG. 13. Magnitude of all level density scalings implemented as part of the global fitting procedure for residual products of proton-induced reactions on arsenic up to $200 \mathrm{MeV}$.

\section{Parameter Adjustment Validation}

As proposed in Fox et al. [21], validation for the suggested parameter changes can be performed by applying the adjusted fit to reaction channels not included in the initial adjustment sensitivity studies in Section IV B. In this work, the validation channels ${ }^{75} \mathrm{As}(\mathrm{p}, \mathrm{x})^{72,70} \mathrm{As}$, ${ }^{68,66} \mathrm{Ge},{ }^{72,66} \mathrm{Ga},{ }^{69 m}, 65 \mathrm{Zn},{ }^{60,58,57,56} \mathrm{Co}$ help test for $\mathrm{cu}-$ mulative cross section effects and far-from-target modeling stability. ${ }^{75} \mathrm{As}(\mathrm{p}, \mathrm{p} 3 \mathrm{n}){ }^{72} \mathrm{As}$ is in fact independently measured here and meets many of the Fox et al. [21] criteria to be included as a residual product channel in the initial parameter optimization. However, since numerous neighboring competing Se and As channels to ${ }^{72}$ As were already incorporated in Section IVB, it became more worthwhile to save ${ }^{72}$ As production as a significant channel for validation. Figure 15 demonstrates the adjusted fit behaviour in these validation channels, where consistently improved predictive power is seen.

It is also possible to further analyze the total nonelastic cross section predictions of the default and adjusted TALYS models, together with the TENDL eval- 


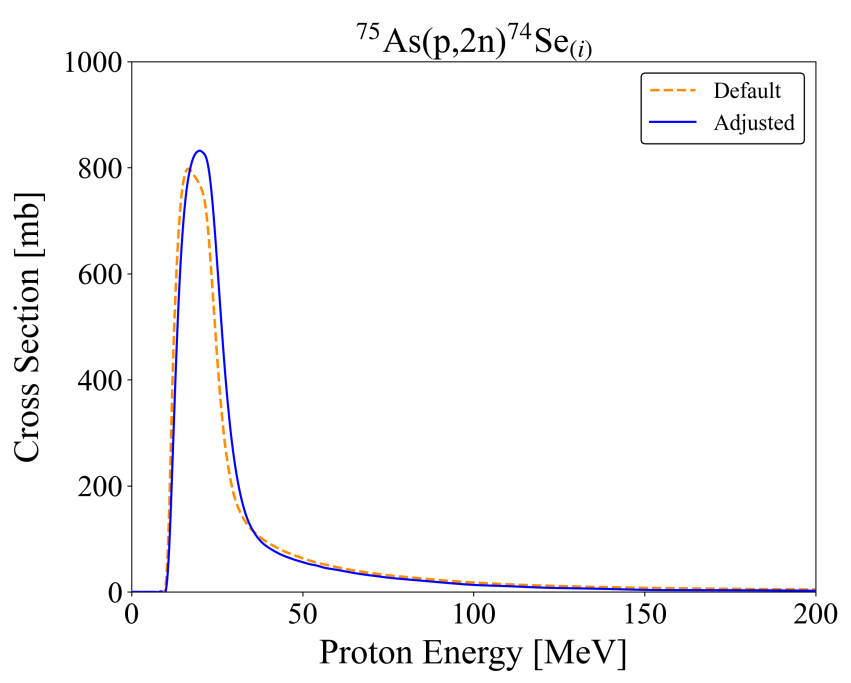

FIG. 14. Performance check for behaviour of the adjusted fit in ${ }^{74} \mathrm{Se}$, the largest unobserved channel from the fitting procedure.

uation (Figure 16). No experimental data points guide the ${ }^{75} \mathrm{As}$ (p,non) predictions, commensurate with the little prior published data for the residual product excitation functions as a whole. Even with the new data results of this paper, due to the unseen reaction products described in Section IV B 1, it is not viable to derive any ${ }^{75} \mathrm{As}(\mathrm{p}$, non $)$ data points from summing the measured cross sections. The adjusted $(p, n o n)$ remains within the TENDL uncertainty band and its increase versus the default is defensible. Specifically, the adjusted (p,non) shares the same shape as the TENDL evaluation and the default prediction, which are based on global fits to other targets, and the increase in magnitude is validated based on changes seen in residual product channels such as ${ }^{73} \mathrm{As}$ in Figure 12d.

A $\chi_{\text {tot }}^{2}$ descriptive metric for comparing the default and adjusted TALYS fits across all presented excitation functions, following the formalism described in Fox et al. [21], is given in Table VI. Both weighting methodologies yield similar results and the adjusted fit is seen to outperform the default prediction. The $\chi_{\text {tot }}^{2}$ values are partially deflated relative to the ${ }^{93} \mathrm{Nb}(\mathrm{p}, \mathrm{x})$ and ${ }^{139} \mathrm{La}(\mathrm{p}, \mathrm{x})$ results in Fox et al. [21] on account of the heavy dependence on the arsenic cross section measurements provided in this work and their associated larger uncertainties (9.0-15\%) stemming from the electroplating process. Consequently, the $\chi_{\text {tot }}^{2}$ results are more usefully viewed as a relative measure between fits rather than as absolute measure of goodness.
TABLE VI. Global $\chi^{2}$ metric describing goodness-of-fit for the default and adjusted TALYS calculations of ${ }^{75} \mathrm{As}(\mathrm{p}, \mathrm{x})$. Low $\chi_{\text {tot }}^{2}$ values, and a case of $\chi_{\text {tot }}^{2}<1.0$, are seen as a function of large weights associated with the measured arsenic data.

\begin{tabular}{lcc}
\hline \hline Weighting Method & Default $\chi_{\text {tot }}^{2}$ & Adjusted $\chi_{\text {tot }}^{2}$ \\
\hline Cumulative $\sigma$ & 2.55 & 0.58 \\
Maximum $\sigma$ & 3.58 & 1.25 \\
\hline \hline
\end{tabular}

\section{Alternative Solutions and Limitations of the Fitting Procedure}

The M2constant $=0.80, \quad$ M2limit $=3.9, \quad$ and M2shift $=0.55$ exciton model adjustments suggested in this paper match the trend of M2constant $<1.0$, M2limit $>1.0$, and M2shift $<1.0$ changes from the ${ }^{93} \mathrm{Nb}(\mathrm{p}, \mathrm{x})$ and ${ }^{139} \mathrm{La}(\mathrm{p}, \mathrm{x})$ fitting cases in Fox et al. [21]. As a result, the same systematic behaviour of a relative decrease for internal transition rates at intermediate proton energies $\left(E_{p}=20-60 \mathrm{MeV}\right)$ in the exciton model as derived from the $\mathrm{Nb}$ and La cases is seen in the As as well. These determined M2 pre-equilibrium adjustments therefore continue to be indicative of a needed global enhancement to the two-component exciton model.

However, due to the mathematical formulation of the exciton model in TALYS, which can be reviewed in detail in Koning and Duijvestijn [119], it has been found that in fact M2constant $<1.0$, M2limit $>1.0$, and M2shift $<1.0$ is not a required condition to generate the systematic behaviour. Instead, numerous sets of (M2constant,M2limit,M2shift) will reproduce the same decrease for internal transition rates and replicate the residual product cross section predictions of Section IV B. For example, both $(2.45,0.7,1.2)$ and $(1.1,2.85$, $0.7)$ satisfy these conditions for the ${ }^{75} \mathrm{As}(\mathrm{p}, \mathrm{x})$ fitting. Thus, the transition rate trend result from Fox et al. [21] is corroborated in this work but the M2 adjustment requirements to create this trend are revised. Moreover, since multiple triplets all predict the expected systematic behaviour for the reaction phase space transitioning between the Hauser-Feshbach and exciton models for nuclear reactions, it is not possible to conclude which triplet is more accurate without more diversified datasets such as particle emission spectra or prompt gamma yields by ${ }^{75} \mathrm{As}(\mathrm{p}, \mathrm{x} \gamma)[114]$. 


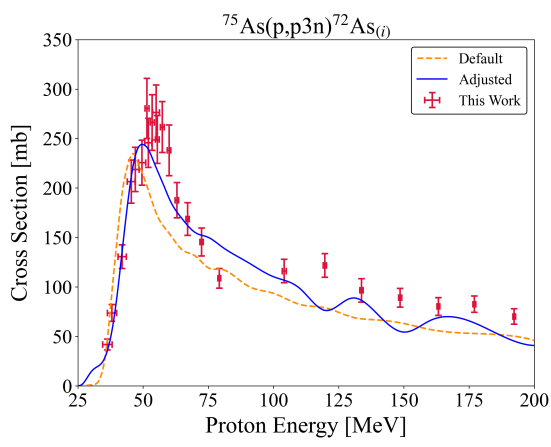

(a)

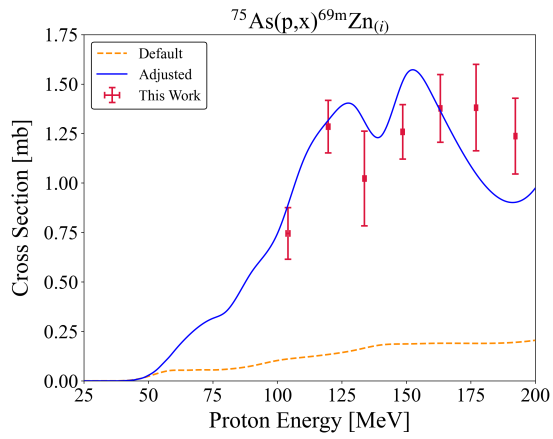

(d)

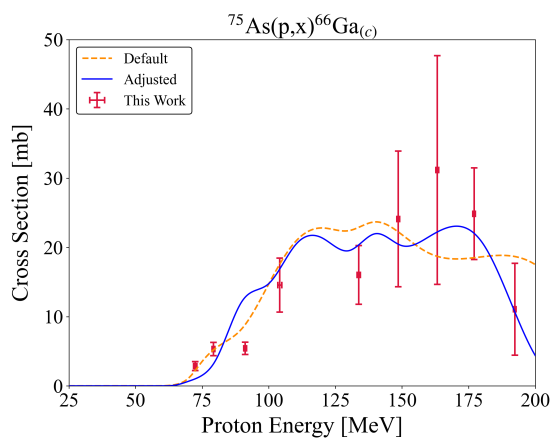

(g)

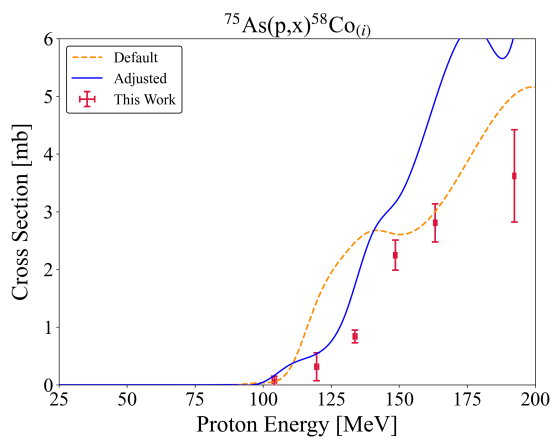

(j)

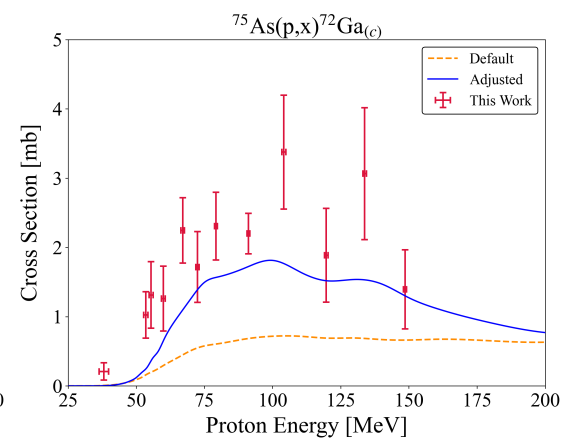

(b)

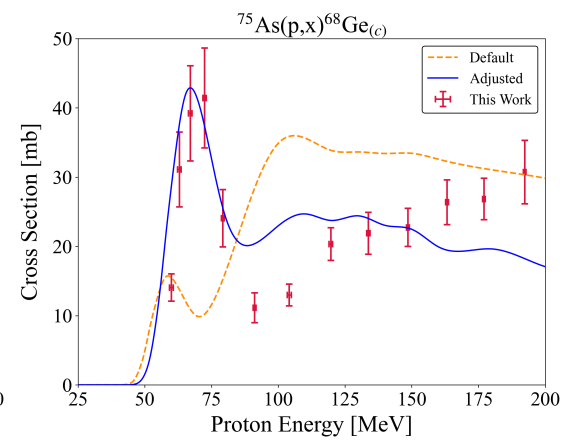

(e)

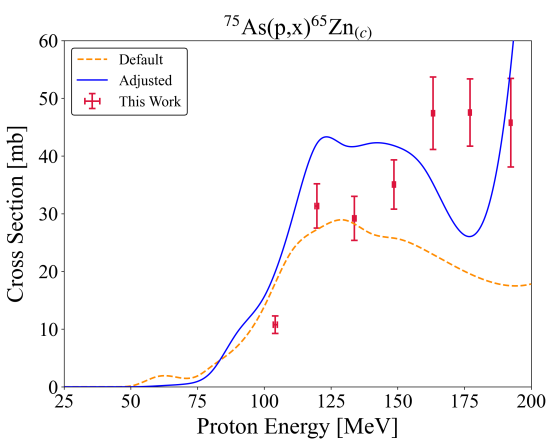

(h)

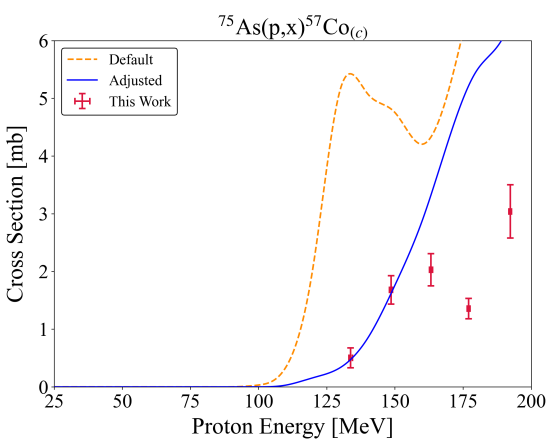

(k)

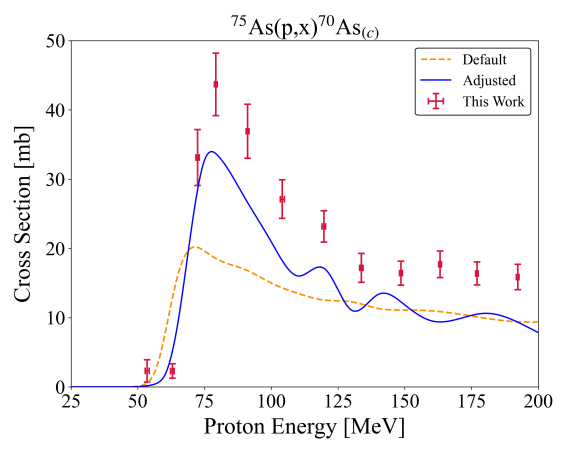

(c)

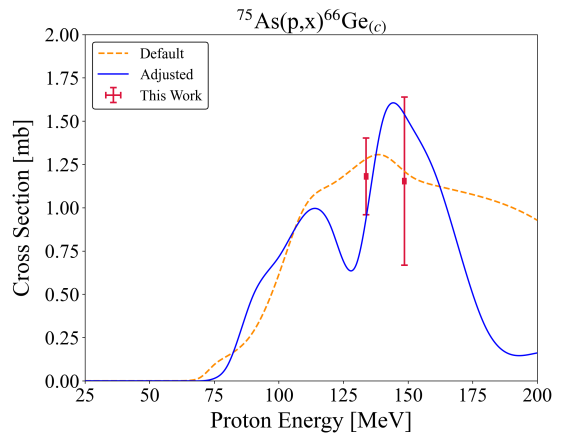

(f)

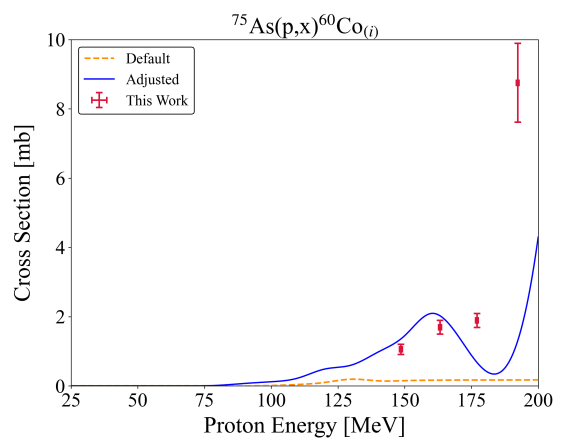

(i)

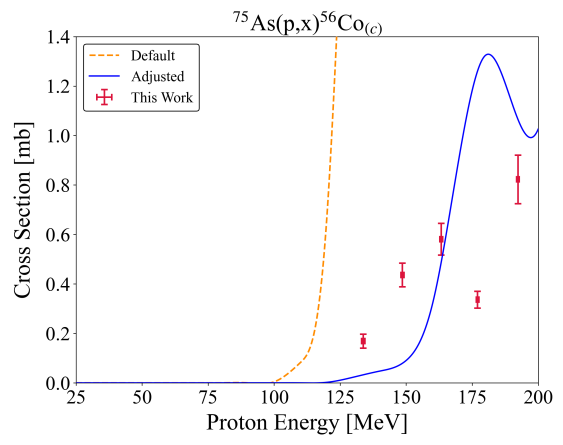

(1)

FIG. 15. TALYS default and adjusted calculations extended to residual products not used in the parameter adjustment sensitivity studies. 


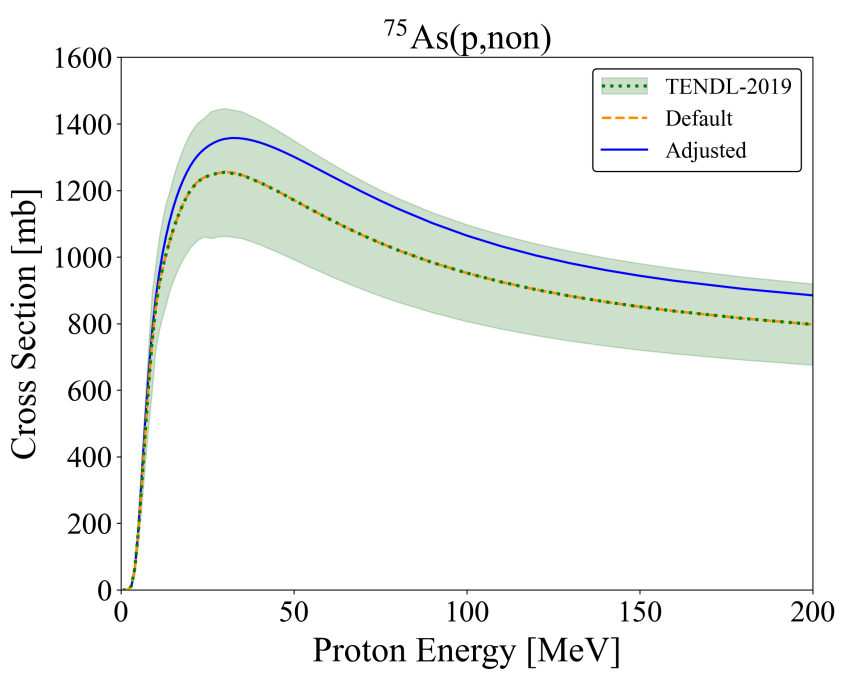

FIG. 16. Comparison of evaluated and theoretical non-elastic cross sections. The filled error bands are associated with the TENDL data.

Indeed, this lack of diversified datasets is the overall limiting factor of the fitting procedure in its current state. The TALYS parameter space is extremely large and the effects of many parameters are hidden from high-energy residual product modeling. Furthermore, the secondary effects from pre-equilibrium, optical model, level density, and coupled-channels changes that are made cannot be deduced without other data types, which detracts from physical insights that can be made about the modeling physics in this work [120]. Prompt gamma data or emission spectra could act to concretely identify certain parameters as well as greatly reduce the remaining parameter space, all creating a more suitable and physical fit solution. Of course, these additional data types would themselves only be able to inform small portions of the incident energy range explored through stackedtarget activation and would not be as useful without the abundance of residual product data. Clearly, continued high-energy reaction measurements of all types are both needed and complementary.

The size of the parameter space is a further limiting element since it leads to local minimum results for the fitting procedure, as was discussed for ${ }^{67} \mathrm{Ga}$ in this work. The implementation of automated searching and/or machine learning could likely mitigate this problem and would be in line with the sentiment of evaluators in the nuclear data community $[22,121]$.

Overall, these shortcomings emphasize that the thought process of the Fox et al. [21] fitting procedure is most relevant, and not every individual result - at this stage, because it principally builds evaluation considerations into nuclear data measurements. This is an important introductory step for an area where no formalism or data existed, as the evolution of this type of thought process better aligns data work and evaluations as a necessary path forward.

\section{CONCLUSIONS}

This work furthers the Tri-laboratory Effort in Nuclear Data by reporting 55 sets of measured ${ }^{75} \mathrm{As}(\mathrm{p}, \mathrm{x})$, ${ }^{\text {nat }} \mathrm{Cu}(\mathrm{p}, \mathrm{x})$, and ${ }^{\text {nat }} \mathrm{Ti}(\mathrm{p}, \mathrm{x})$ residual product cross sections between 35 and $200 \mathrm{MeV}$. The measured data most notably include the first cross section results for ${ }^{75} \mathrm{As}(\mathrm{p}, \mathrm{x}){ }^{68} \mathrm{Ge}$ and the best characterized excitation function of ${ }^{75} \mathrm{As}(\mathrm{p}, \mathrm{x}){ }^{72} \mathrm{Se}$ to-date, which are important for the production of the ${ }^{68} \mathrm{Ge} /{ }^{68} \mathrm{Ga}$ and ${ }^{72} \mathrm{Se} /{ }^{72} \mathrm{As} \mathrm{PET}$ generator systems.

We have additionally continued to develop the Fox et al. [21] formalism for high-energy reaction modeling using the newly available measured ${ }^{75} \mathrm{As}(\mathrm{p}, \mathrm{x})$ data. The modeling study in this paper corroborated the preequilibrium exciton model findings presented in Fox et al. [21] surrounding the transition between the compound and pre-equilibrium regions in TALYS. Furthermore, we provided an in-depth discussion on the limitations to modeling predictive power caused by the lack of level density knowledge for nuclei off of stability.

This paper merges experimental work and evaluation techniques for high-energy charged-particle isotope production in a continuance of the initial analysis of this kind. The consideration of these different aspects of the nuclear data pipeline together is a priority moving forwards that will benefit future data compilation, evaluation, and application.

The $\gamma$-ray spectra and all other raw data created during this research are openly available at http://doi. org/10.5281/zenodo.4648950 [122]. On publication, the experimentally determined cross sections will be uploaded to the EXFOR database.

\section{ACKNOWLEDGMENTS}

This research was supported by the U.S. Department of Energy Isotope Program, managed by the Office of Science for Isotope R\&D and Production, and was carried out under Lawrence Berkeley National Laboratory (Contract No. DE-AC02-05CH11231), Los Alamos National Laboratory (Contract No. 89233218CNA000001), and Brookhaven National Laboratory (Contract No. DEAC02-98CH10886). The authors acknowledge the assistance and support of Brien Ninemire, Scott Small, Nick Brickner, Devin Thatcher, and all the rest of the operations, research, and facilities staff of the LBNL 88-Inch Cyclotron. We also thank David Reass and Mike Connors at LANSCE-IPF, the LANL C-NR Countroom operators, and the LANSCE Accelerator Operations staff. The authors acknowledge Deepak Raparia, head of the Pre-Injector Systems group at CAD-BNL, for LINAC beam tuning for the experiment and all members of the BNL Medical Isotope Research and Production group for their assistance. We are grateful to Patrick Sullivan and John Aloi of the BNL Radiological Control Division for the Health Physics support. Sumanta Nayak is acknowledged for the engineering and Frank Naase for the IT support. 


\section{Appendix A: Additional LANL and BNL Target Stack Information}

Details of the stacked-targets irradiated at LANL and BNL are given in Tables VII and VIII, taken directly from Fox et al. [21].

TABLE VII: Target stack design for irradiation at IPF. The proton beam initially hits the stainless steel plate (SS-SN1) after passing through the upstream Inconel beam entrance window, a water cooling channel, and the target box aluminum window. The thickness and areal density measurements are prior to any application of the variance minimization techniques described in this work.

\begin{tabular}{|c|c|c|c|}
\hline Target Layer & $\begin{array}{c}\text { Thickness } \\
{[\mu \mathrm{m}]}\end{array}$ & $\begin{array}{c}\text { Areal } \\
\text { Density } \\
{\left[\mathrm{mg} / \mathrm{cm}^{2}\right]}\end{array}$ & $\begin{array}{c}\text { Areal } \\
\text { Density } \\
\text { Uncertainty } \\
{[\%]}\end{array}$ \\
\hline SS-SN1 Profile Monitor & 130.0 & 100.12 & 0.07 \\
\hline Al-SN1 & 27.33 & 7.51 & 0.21 \\
\hline Nb-SN1 & 25.75 & 23.08 & 0.12 \\
\hline As-SN1 & 4.27 & 2.45 & 8.2 \\
\hline Ti-SN1 & 25.00 & 11.265 & 1.0 \\
\hline $\mathrm{Cu}-\mathrm{SN} 1$ & 24.33 & 19.04 & 0.13 \\
\hline Al Degrader 01 & 6307.0 & 1702.89 & 0.001 \\
\hline Al-SN2 & 26.67 & 7.58 & 0.32 \\
\hline $\mathrm{Nb}-\mathrm{SN} 2$ & 24.75 & 22.67 & 0.08 \\
\hline As-SN2 & 4.30 & 2.46 & 8.3 \\
\hline Ti-SN2 & 25.00 & 11.265 & 1.0 \\
\hline $\mathrm{Cu}-\mathrm{SN} 2$ & 24.00 & 18.90 & 0.36 \\
\hline Al Degrader 02 & 3185.5 & 860.09 & 0.02 \\
\hline Al-SN3 & 26.67 & 7.38 & 0.22 \\
\hline Nb-SN3 & 24.50 & 22.83 & 0.03 \\
\hline As-SN3 & 3.62 & 2.07 & 9.0 \\
\hline Ti-SN3 & 25.00 & 11.265 & 1.0 \\
\hline $\mathrm{Cu}-\mathrm{SN} 3$ & 23.33 & 19.38 & 0.11 \\
\hline Al Degrader 03 & 2304.5 & 622.22 & 0.06 \\
\hline Al-SN4 & 28.00 & 7.34 & 0.18 \\
\hline $\mathrm{Nb}-\mathrm{SN} 4$ & 25.50 & 22.57 & 0.16 \\
\hline As-SN4 & 3.54 & 2.03 & 9.2 \\
\hline Ti-SN4 & 25.00 & 11.265 & 1.0 \\
\hline $\mathrm{Cu}-\mathrm{SN} 4$ & 24.67 & 19.24 & 0.11 \\
\hline Al Degrader 04 & 1581.3 & 426.94 & 0.04 \\
\hline Al-SN5 & 27.00 & 7.48 & 0.44 \\
\hline Nb-SN5 & 24.75 & 22.78 & 0.12 \\
\hline As-SN5 & 3.90 & 2.23 & 8.7 \\
\hline Ti-SN5 & 25.00 & 11.265 & 1.0 \\
\hline $\mathrm{Cu}-\mathrm{SN} 5$ & 25.00 & 19.09 & 0.17 \\
\hline Al Degrader 05 & 1033.8 & 279.11 & 0.06 \\
\hline Al-SN6 & 28.67 & 7.44 & 0.25 \\
\hline $\mathrm{Nb}-\mathrm{SN} 6$ & 25.25 & 22.80 & 0.08 \\
\hline As-SN6 & 3.11 & 1.78 & 10 \\
\hline
\end{tabular}

Continued on next page
TABLE VII - cont.

\begin{tabular}{|c|c|c|c|}
\hline Target Layer & $\begin{array}{c}\text { Thickness } \\
{[\mu \mathrm{m}]}\end{array}$ & $\begin{array}{c}\text { Areal } \\
\text { Density } \\
{\left[\mathrm{mg} / \mathrm{cm}^{2}\right]}\end{array}$ & $\begin{array}{c}\text { Areal } \\
\text { Density } \\
\text { Uncertainty } \\
{[\%]}\end{array}$ \\
\hline Ti-SN6 & 25.00 & 11.265 & 1.0 \\
\hline $\mathrm{Cu}-\mathrm{SN} 6$ & 24.33 & 19.50 & 0.16 \\
\hline Al Degrader 06 & 834.8 & 225.38 & 0.22 \\
\hline Al-SN7 & 28.33 & 7.56 & 0.15 \\
\hline $\mathrm{Nb}-\mathrm{SN} 7$ & 25.50 & 22.62 & 0.06 \\
\hline As-SN7 & 2.79 & 1.59 & 9.2 \\
\hline Ti-SN7 & 25.00 & 11.265 & 1.0 \\
\hline $\mathrm{Cu}-\mathrm{SN} 7$ & 23.67 & 18.79 & 0.04 \\
\hline Al Degrader 07 & 513.5 & 138.65 & 0.10 \\
\hline Al-SN8 & 27.67 & 7.56 & 0.10 \\
\hline $\mathrm{Nb}-\mathrm{SN} 8$ & 25.50 & 22.95 & 0.45 \\
\hline As-SN8 & 2.20 & 1.26 & 9.0 \\
\hline Ti-SN8 & 25.00 & 11.265 & 1.0 \\
\hline $\mathrm{Cu}-\mathrm{SN} 8$ & 24.00 & 19.06 & 0.23 \\
\hline Al Degrader 08 & 517.3 & 139.66 & 0.43 \\
\hline Al-SN9 & 27.00 & 7.47 & 0.36 \\
\hline Nb-SN9 & 25.00 & 22.53 & 0.24 \\
\hline As-SN9 & 2.57 & 1.47 & 9.9 \\
\hline Ti-SN9 & 25.00 & 11.265 & 1.0 \\
\hline $\mathrm{Cu}-\mathrm{SN} 9$ & 26.33 & 19.19 & 0.12 \\
\hline Al Degrader 09 & 517.8 & 139.79 & 0.09 \\
\hline Al-SN10 & 28.00 & 7.41 & 0.17 \\
\hline Nb-SN10 & 24.75 & 22.82 & 0.02 \\
\hline As-SN10 & 1.94 & 1.11 & 10 \\
\hline Ti-SN10 & 25.00 & 11.265 & 1.0 \\
\hline $\mathrm{Cu}-\mathrm{SN} 10$ & 25.67 & 18.87 & 0.18 \\
\hline SS-SN10 Profile Monitor & 130.0 & 100.12 & 0.07 \\
\hline
\end{tabular}


TABLE VIII. Target stack design for irradiation at BLIP. The proton beam initially hits the stainless steel plate after passing through the upstream beam windows, water cooling channels, and target box aluminum window. The thickness and areal density measurements are prior to any application of the variance minimization techniques described in this work.

\begin{tabular}{|c|c|c|c|}
\hline Target Layer & $\begin{array}{c}\text { Thickness } \\
{[\mu \mathrm{m}]}\end{array}$ & $\begin{array}{c}\text { Areal } \\
\text { Density } \\
{\left[\mathrm{mg} / \mathrm{cm}^{2}\right]}\end{array}$ & $\begin{array}{c}\text { Areal } \\
\text { Density } \\
\text { Uncertainty } \\
{[\%]}\end{array}$ \\
\hline SS Profile Monitor & 120.2 & 95.16 & 0.58 \\
\hline $\mathrm{Cu}-\mathrm{SN} 1$ & 26.00 & 22.34 & 0.10 \\
\hline Nb-SN1 & 25.75 & 22.75 & 0.25 \\
\hline As-SN1 & 1.89 & 1.08 & 9.9 \\
\hline Ti-SN1 & 25.00 & 11.265 & 1.0 \\
\hline $\mathrm{Cu}$ Degrader 01 & 5261.1 & 4708.07 & 0.02 \\
\hline $\mathrm{Cu}-\mathrm{SN} 2$ & 26.75 & 22.41 & 0.11 \\
\hline $\mathrm{Nb}-\mathrm{SN} 2$ & 24.75 & 22.91 & 0.19 \\
\hline As-SN2 & 2.94 & 1.68 & 9.0 \\
\hline Ti-SN2 & 25.00 & 11.265 & 1.0 \\
\hline $\mathrm{Cu}$ Degrader 02 & 4490.7 & 4018.99 & 0.04 \\
\hline $\mathrm{Cu}-\mathrm{SN} 3$ & 26.50 & 22.26 & 0.05 \\
\hline Nb-SN3 & 24.00 & 22.67 & 0.31 \\
\hline As-SN3 & 3.06 & 1.75 & 10 \\
\hline Ti-SN3 & 25.00 & 11.265 & 1.0 \\
\hline $\mathrm{Cu}$ Degrader 03 & 4501.8 & 4028.84 & 0.03 \\
\hline $\mathrm{Cu}-\mathrm{SN} 4$ & 26.00 & 22.29 & 0.15 \\
\hline Nb-SN4 & 24.75 & 22.70 & 0.23 \\
\hline As-SN4 & 4.85 & 2.78 & 9.9 \\
\hline Ti-SN4 & 25.00 & 11.265 & 1.0 \\
\hline $\mathrm{Cu}$ Degrader 04 & 4243.9 & 3797.96 & 0.03 \\
\hline Cu-SN5 & 25.50 & 22.35 & 0.04 \\
\hline Nb-SN5 & 25.00 & 22.54 & 0.12 \\
\hline As-SN5 & 7.26 & 4.15 & 12 \\
\hline Ti-SN5 & 25.00 & 11.265 & 1.0 \\
\hline $\mathrm{Cu}$ Degrader 05 & 3733.8 & 3341.56 & 0.03 \\
\hline $\mathrm{Cu}-\mathrm{SN} 6$ & 26.25 & 22.34 & 0.08 \\
\hline Nb-SN6 & 25.00 & 22.36 & 0.24 \\
\hline As-SN6 & 4.93 & 2.82 & 9.0 \\
\hline Ti-SN6 & 25.00 & 11.265 & 1.0 \\
\hline $\mathrm{Cu}$ Degrader 06 & 3783.0 & 3385.41 & 0.04 \\
\hline $\mathrm{Cu}-\mathrm{SN} 7$ & 25.75 & 22.26 & 0.09 \\
\hline Nb-SN7 & 25.75 & 22.62 & 0.10 \\
\hline As-SN7 & 12.62 & 7.22 & 9.3 \\
\hline Ti-SN7 & 25.00 & 11.265 & 1.0 \\
\hline
\end{tabular}

\section{Appendix B: Proton Current Variance Minimization}

The applied variance minimization technique for the LBNL irradiation is summarized in Figure 17. A $4.23 \%$ increase to stopping power in simulations, implemented through an equivalent increase to degraders' effective density in the stack, best reduced proton fluence measurement disagreements between different monitor channels in each energy compartment. This is in general agreement with results of past stacked-target work that have shown a needed modest positive enhancement to the stopping power of $+2-5 \%$ [23-25].

The associated proton flux spectrum propagating through the stack, after variance minimization, is provided in Figure 18. The energy assignments for each foil in a stack are then the flux-averaged energies from the spectrum with uncertainties per foil taken as the full width at half maximum.

This same calculation methodology can be reviewed in detail for the LANL and BNL stacks in Fox et al. [21]

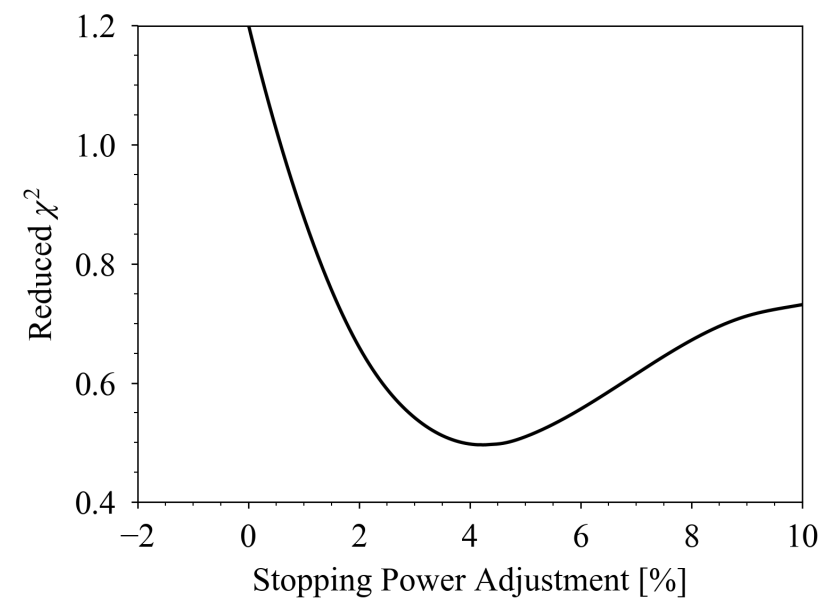

FIG. 17. Result of $\chi^{2}$ optimization used in the variance minimization of the global linear fit to the monitor fluence data, indicating a required increase to stopping power in transport simulations.

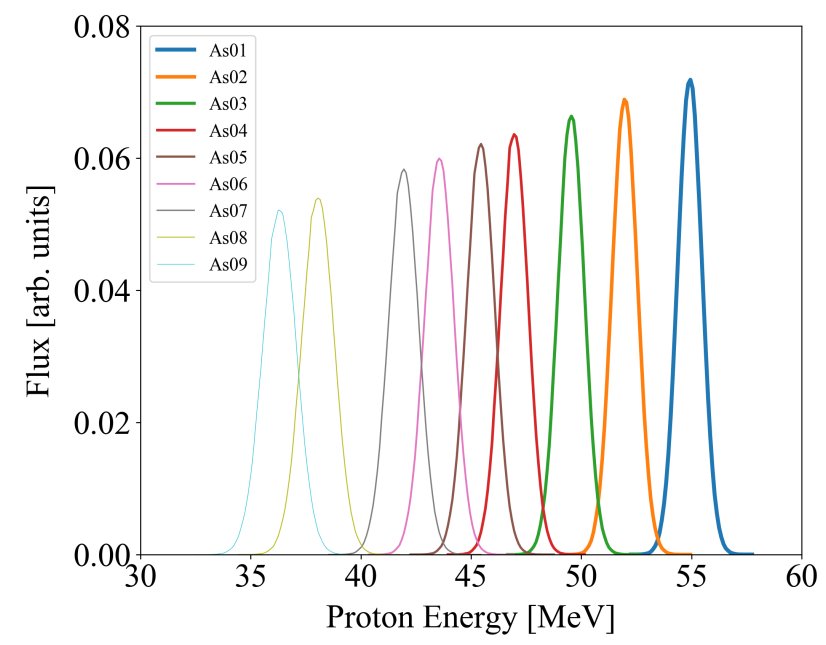

FIG. 18. Visualization of the calculated proton energy spectrum for each arsenic target in the LBNL stack, following variance minimization. 


\section{Appendix C: Measured Excitation Functions}

Plots of extracted cross sections in this work are given (Figures 19-69) with reference to existing literature data, TENDL-2019, and reaction modeling codes TALYS-1.9, EMPIRE-3.2.3, CoH-3.5.3, and ALICE-20 using default parameters [7, 23-25, 33, 39-79]. Subscripts $(i)$ and $(c)$ in figure titles indicate independent and cumulative cross sections, respectively.

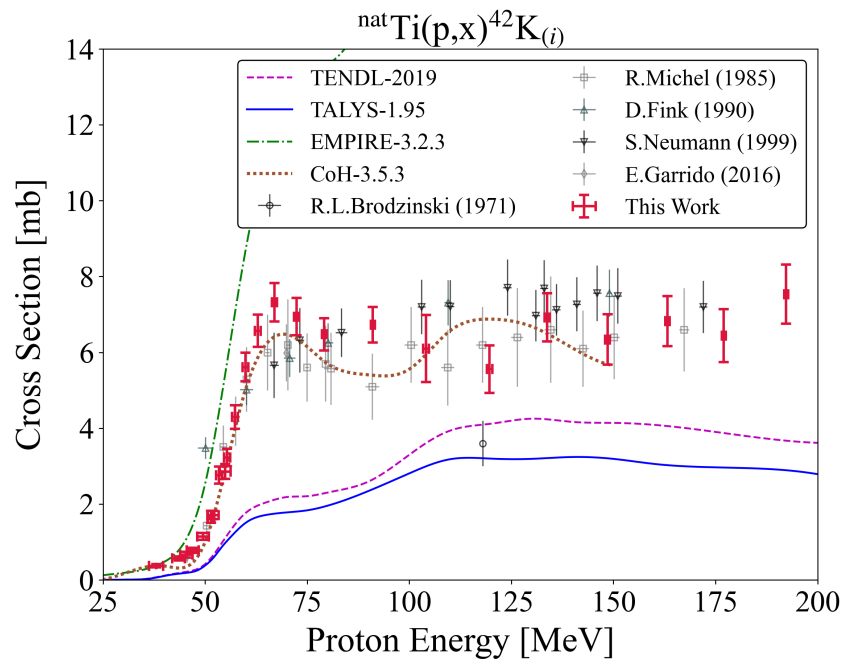

FIG. 19. Experimental and theoretical cross sections for ${ }^{42} \mathrm{~K}$ production.

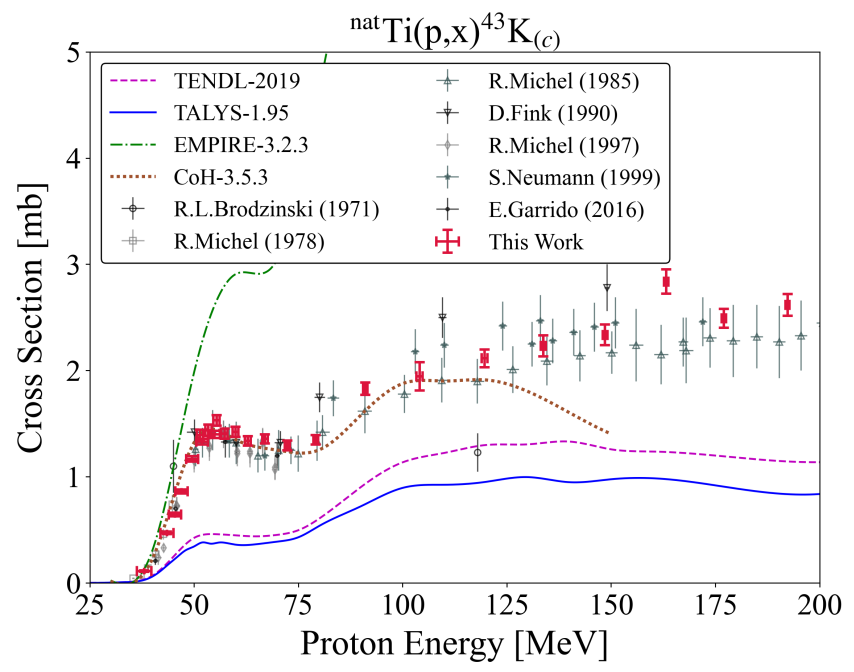

FIG. 20. Experimental and theoretical cross sections for ${ }^{43} \mathrm{~K}$ production.

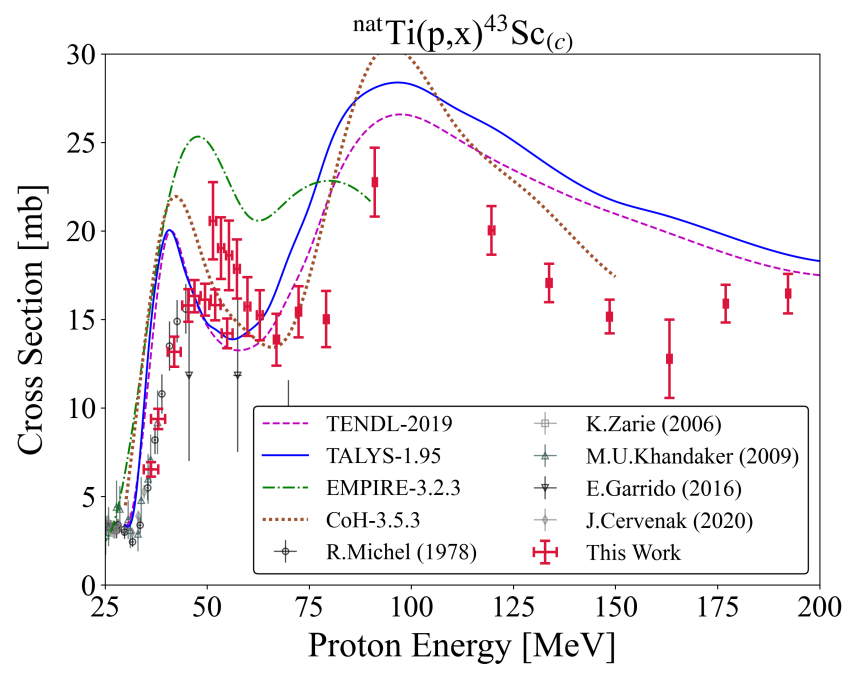

FIG. 21. Experimental and theoretical cross sections for ${ }^{43} \mathrm{Sc}$ production. 


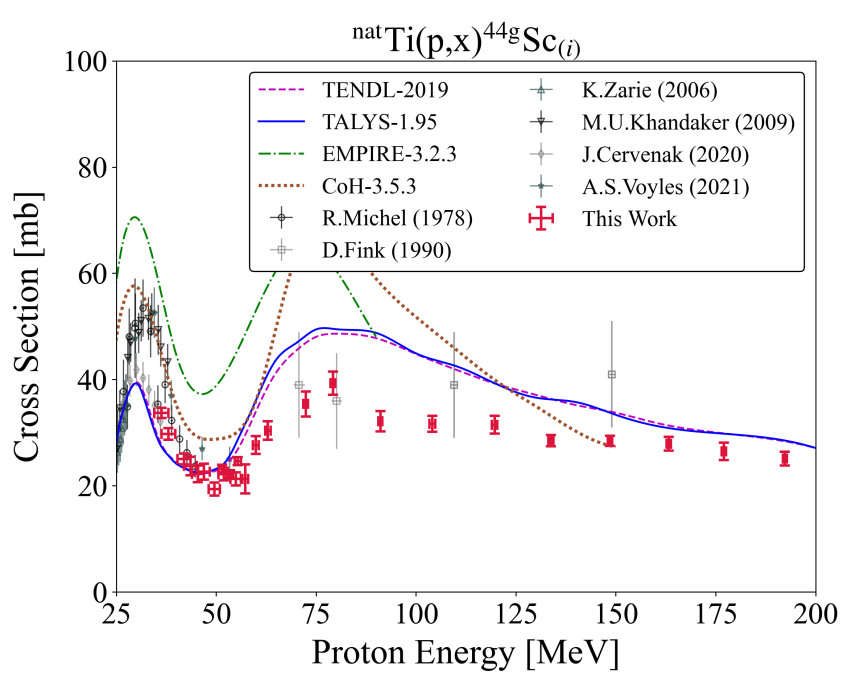

FIG. 22. Experimental and theoretical cross sections for ${ }^{44 \mathrm{~g}} \mathrm{Sc}$ production.

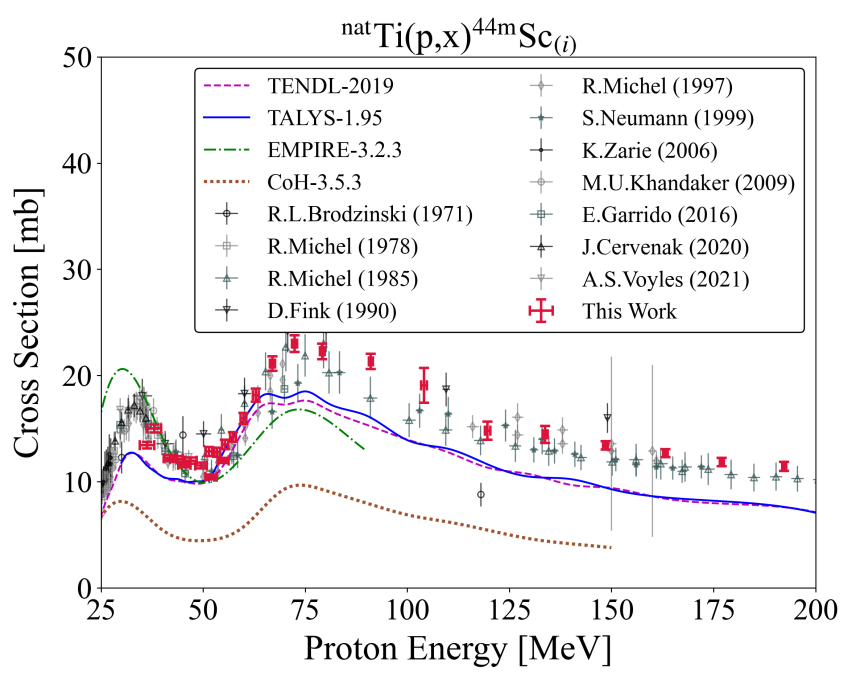

FIG. 23. Experimental and theoretical cross sections for ${ }^{44 \mathrm{~m}} \mathrm{Sc}$ production.

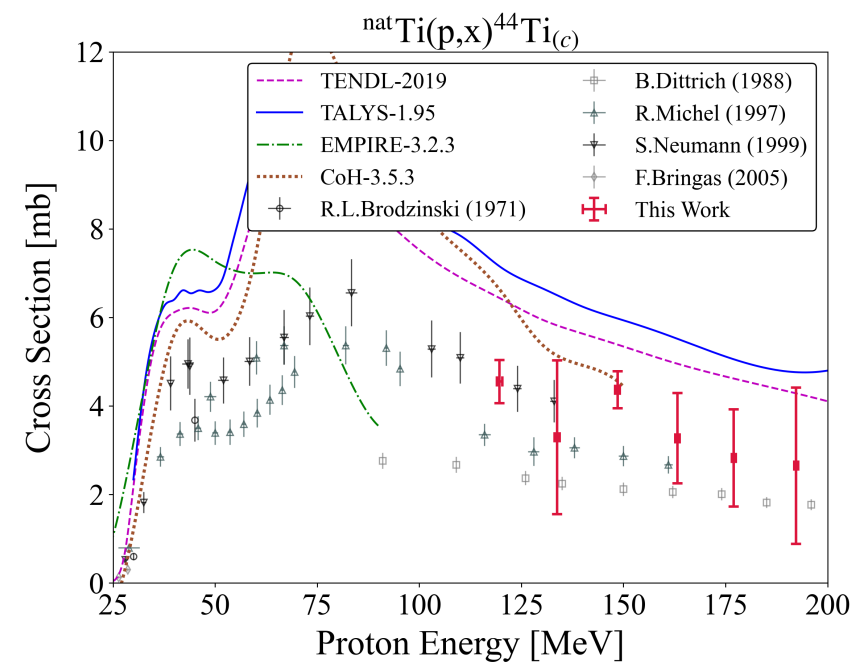

FIG. 24. Experimental and theoretical cross sections for ${ }^{44} \mathrm{Ti}$ production.

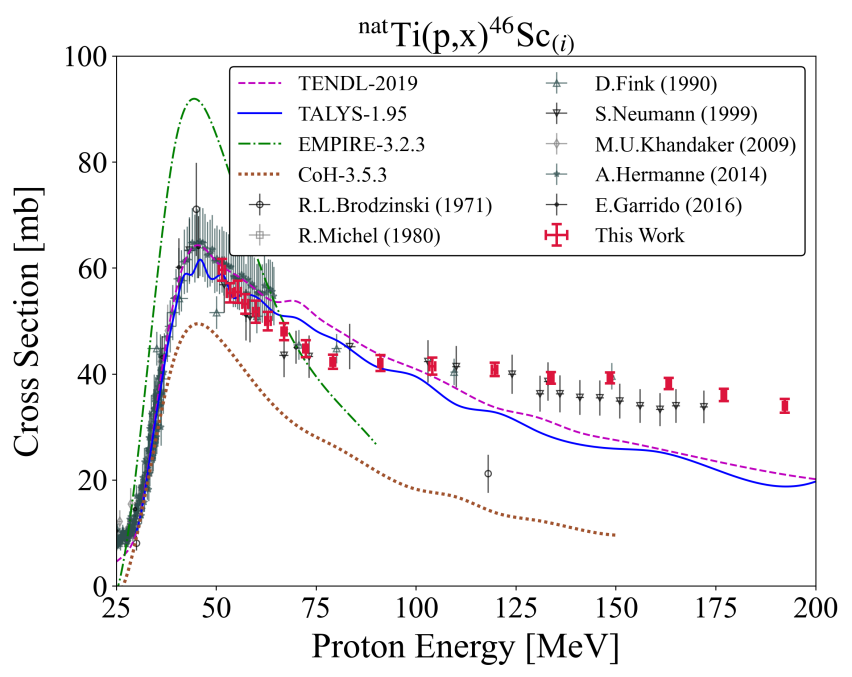

FIG. 25. Experimental and theoretical cross sections for ${ }^{46} \mathrm{Sc}$ production.

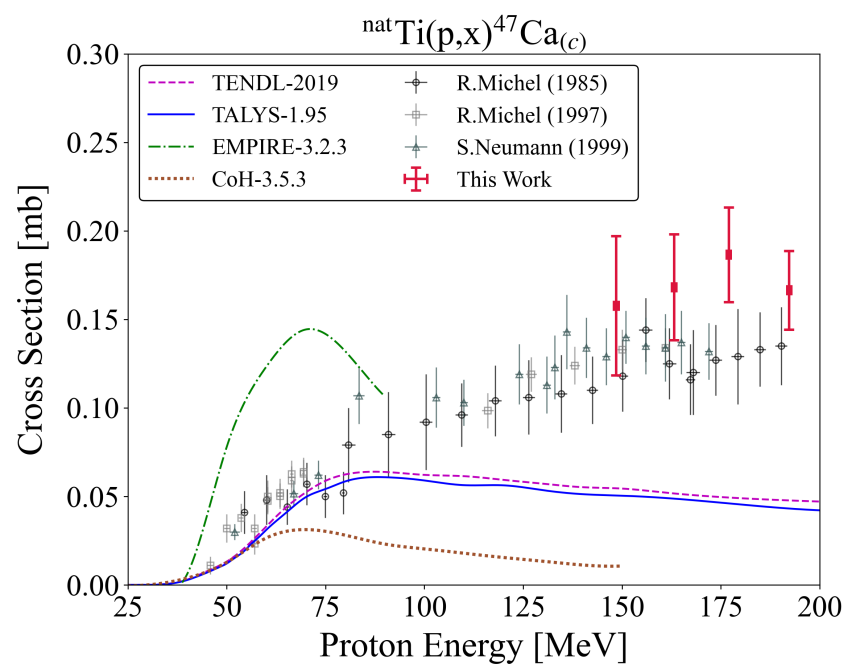

FIG. 26. Experimental and theoretical cross sections for ${ }^{47} \mathrm{Ca}$ production.

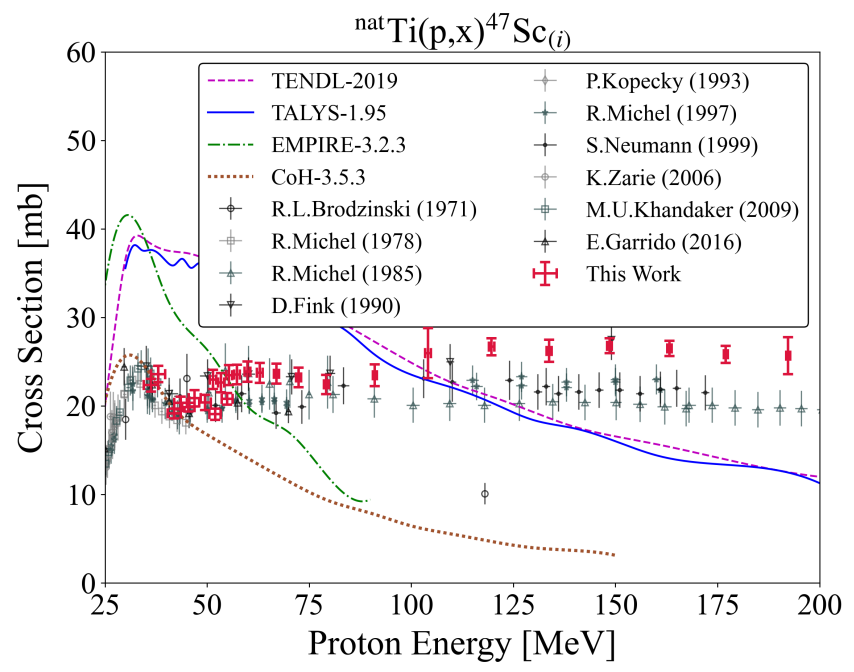

FIG. 27. Experimental and theoretical cross sections for ${ }^{47} \mathrm{Sc}$ production. 


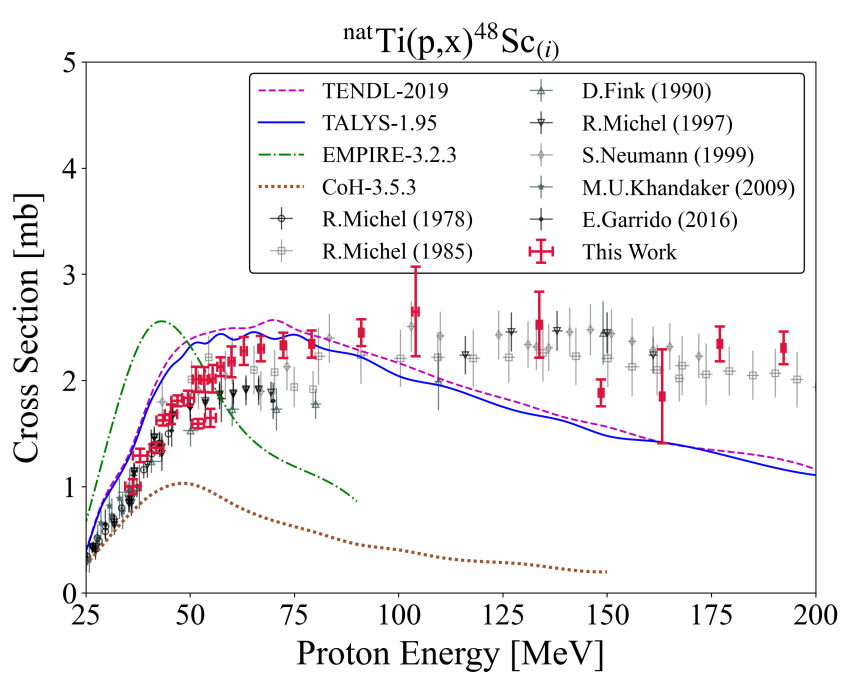

FIG. 28. Experimental and theoretical cross sections for ${ }^{48} \mathrm{Sc}$ production.

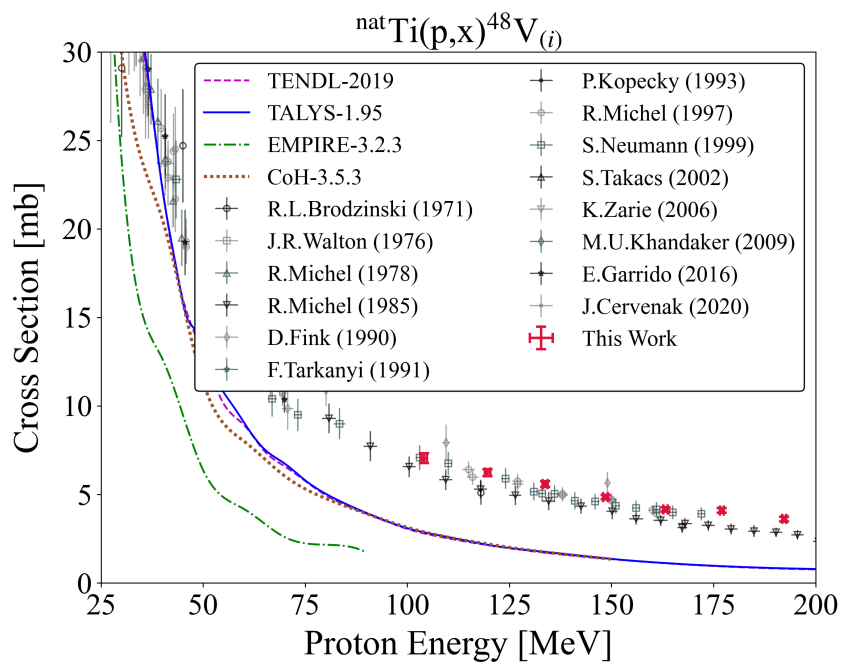

FIG. 29. Experimental and theoretical cross sections for ${ }^{48} \mathrm{~V}$ production.

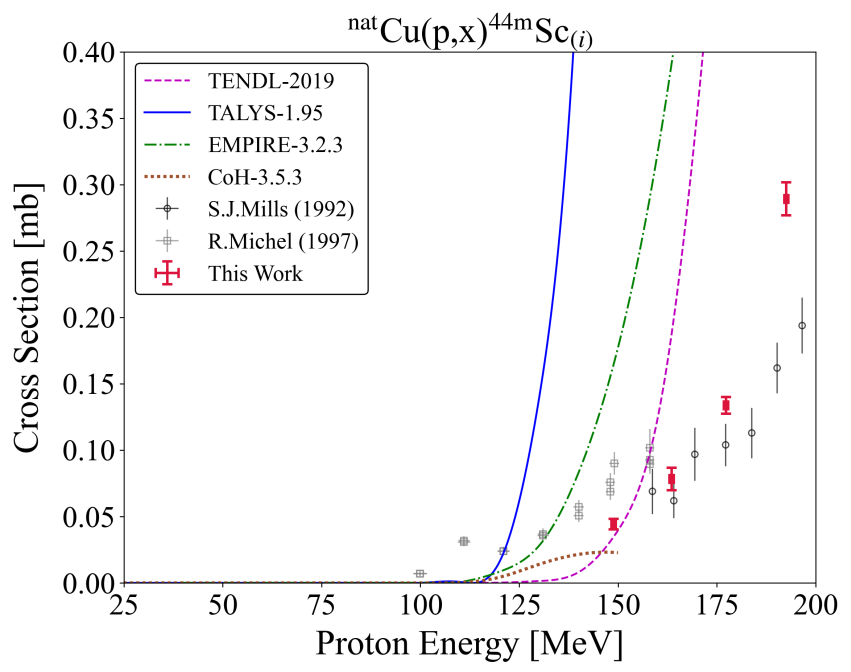

FIG. 30. Experimental and theoretical cross sections for ${ }^{44 \mathrm{~m}} \mathrm{Sc}$ production.

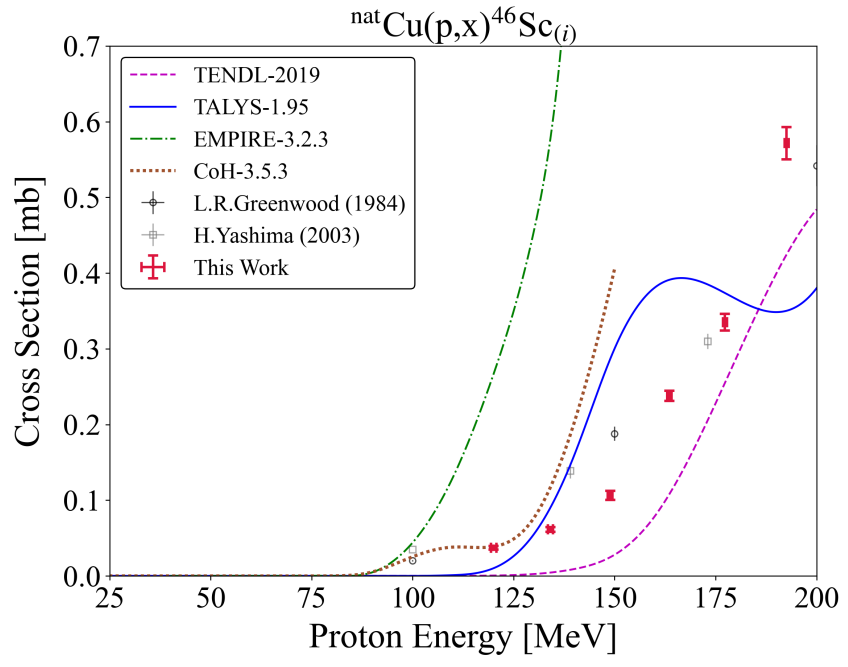

FIG. 31. Experimental and theoretical cross sections for ${ }^{46} \mathrm{Sc}$ production.

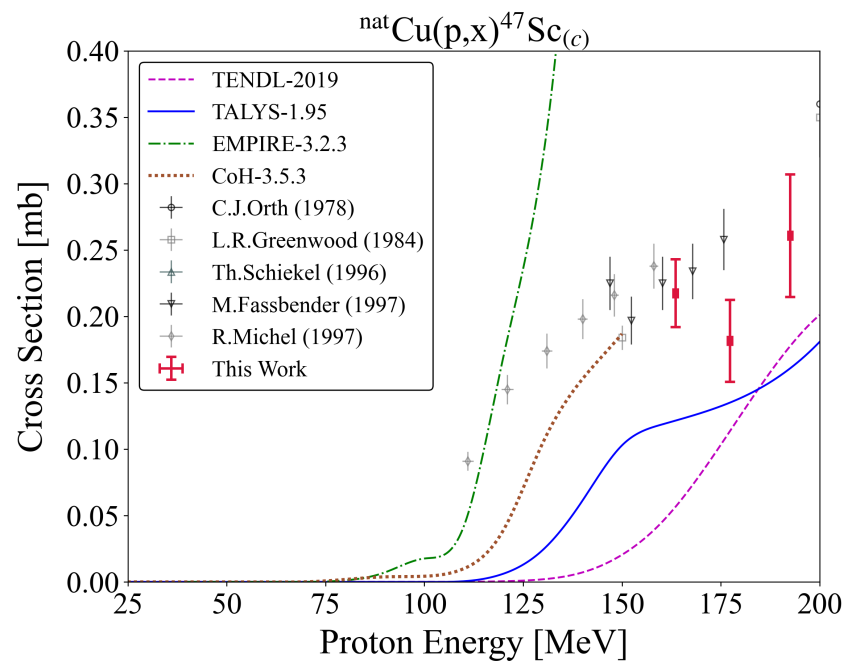

FIG. 32. Experimental and theoretical cross sections for ${ }^{47} \mathrm{Sc}$ production.

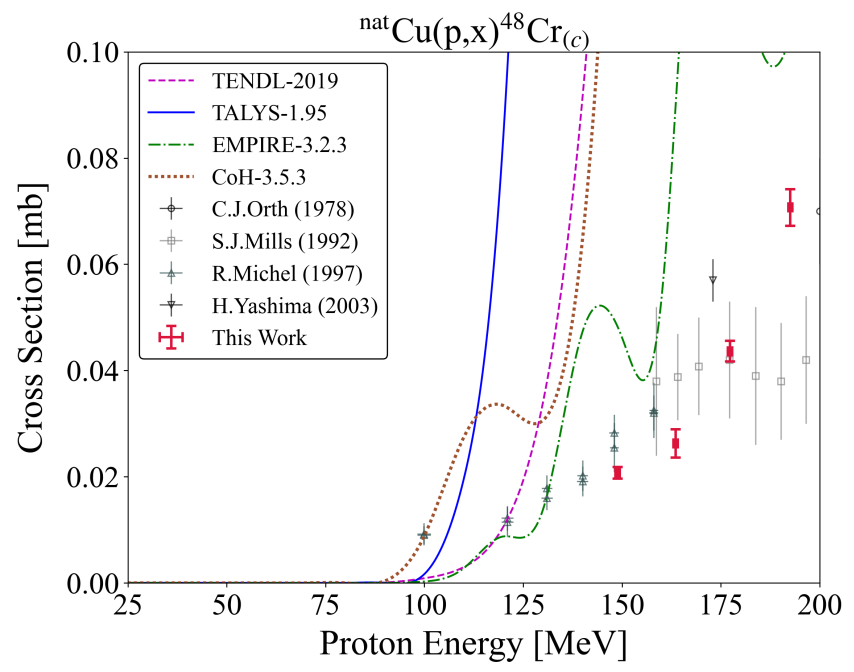

FIG. 33. Experimental and theoretical cross sections for ${ }^{48} \mathrm{Cr}$ production. 


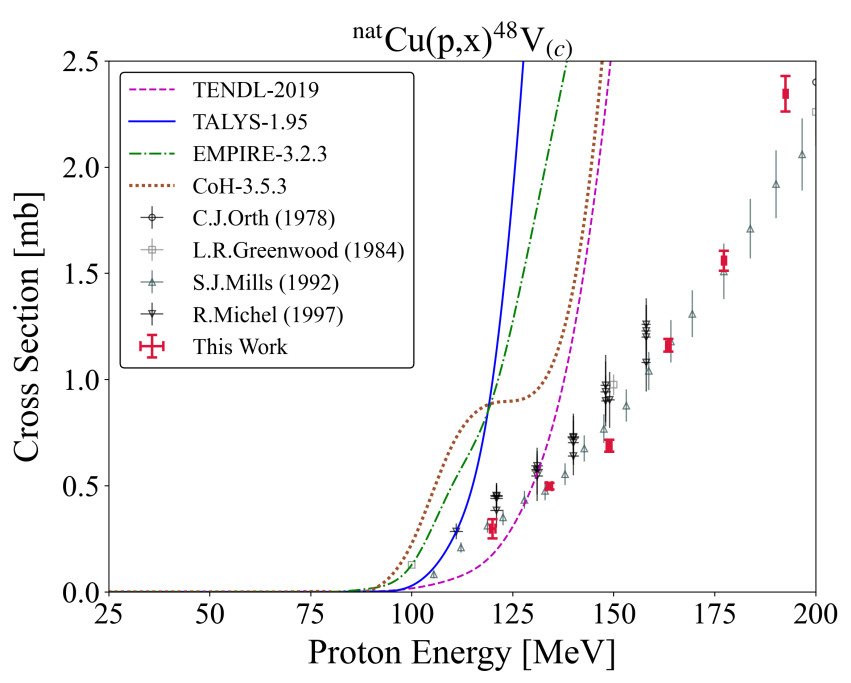

FIG. 34. Experimental and theoretical cross sections for ${ }^{48} \mathrm{~V}$ production.

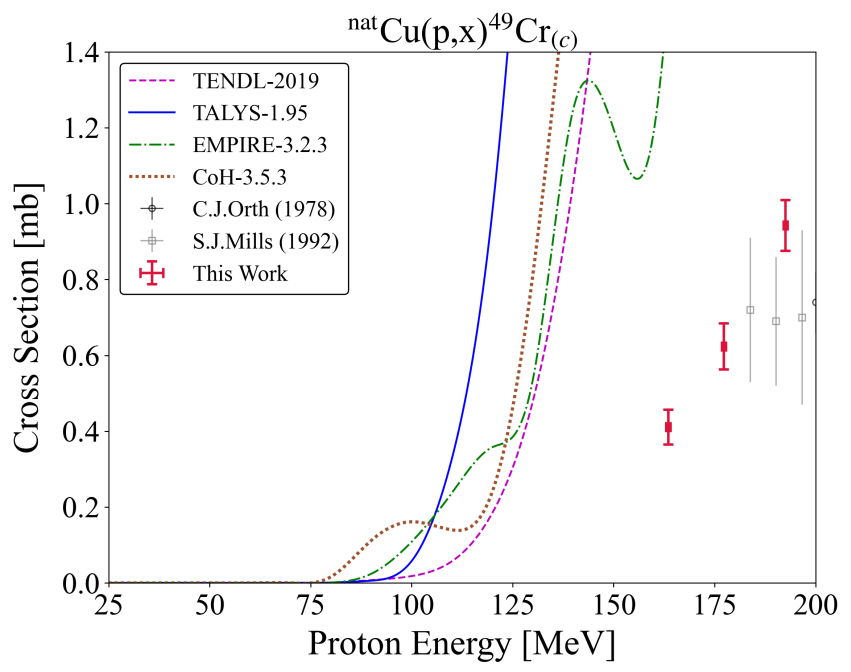

FIG. 35. Experimental and theoretical cross sections for ${ }^{49} \mathrm{Cr}$ production.

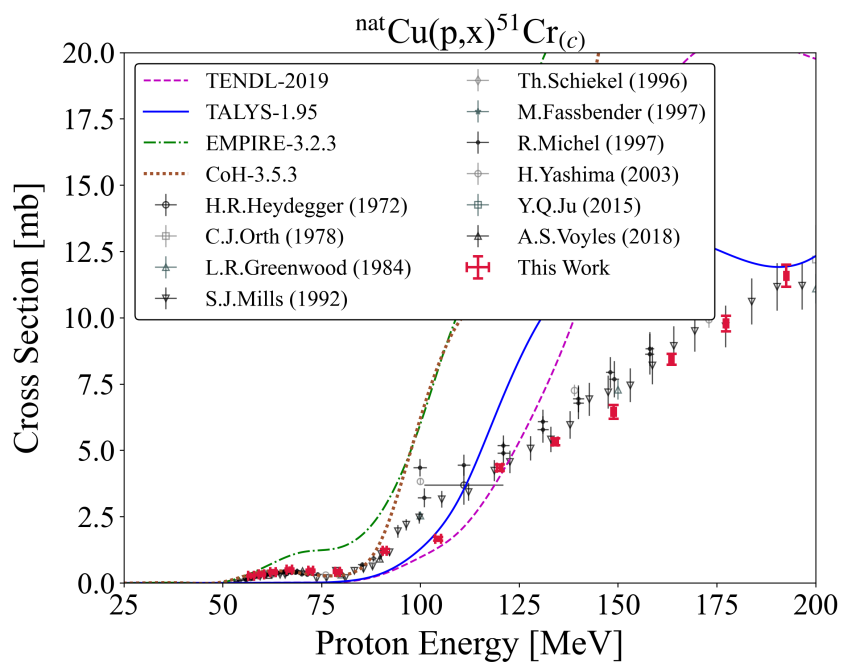

FIG. 36. Experimental and theoretical cross sections for ${ }^{51} \mathrm{Cr}$ production.

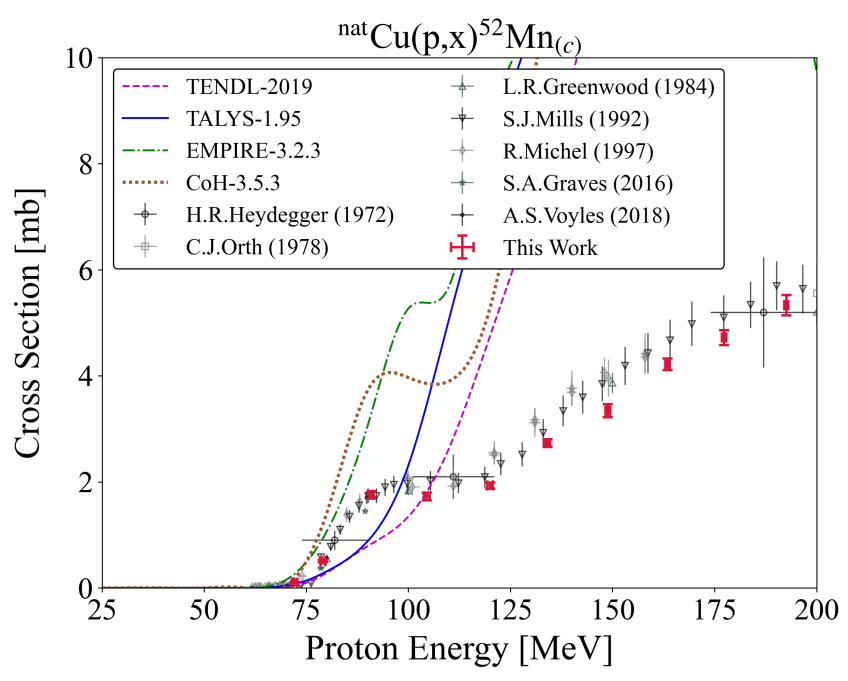

FIG. 37. Experimental and theoretical cross sections for ${ }^{52} \mathrm{Mn}$ production.

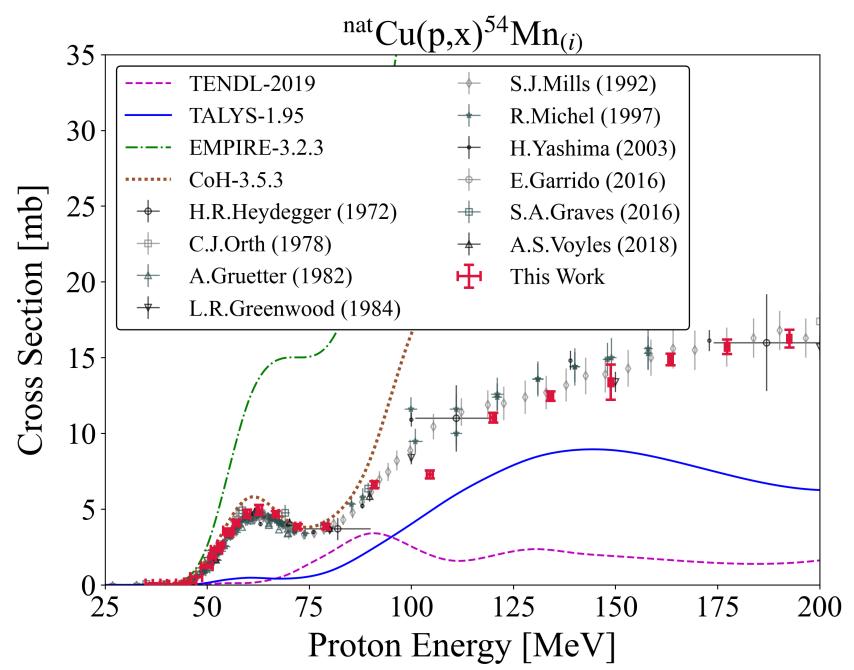

FIG. 38. Experimental and theoretical cross sections for ${ }^{54} \mathrm{Mn}$ production.

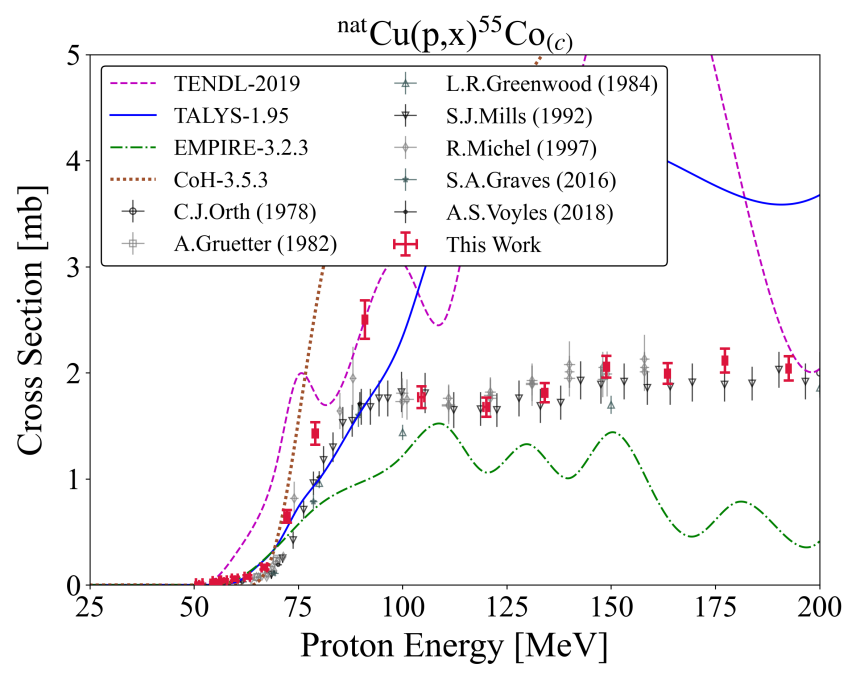

FIG. 39. Experimental and theoretical cross sections for ${ }^{55} \mathrm{Co}$ production. 


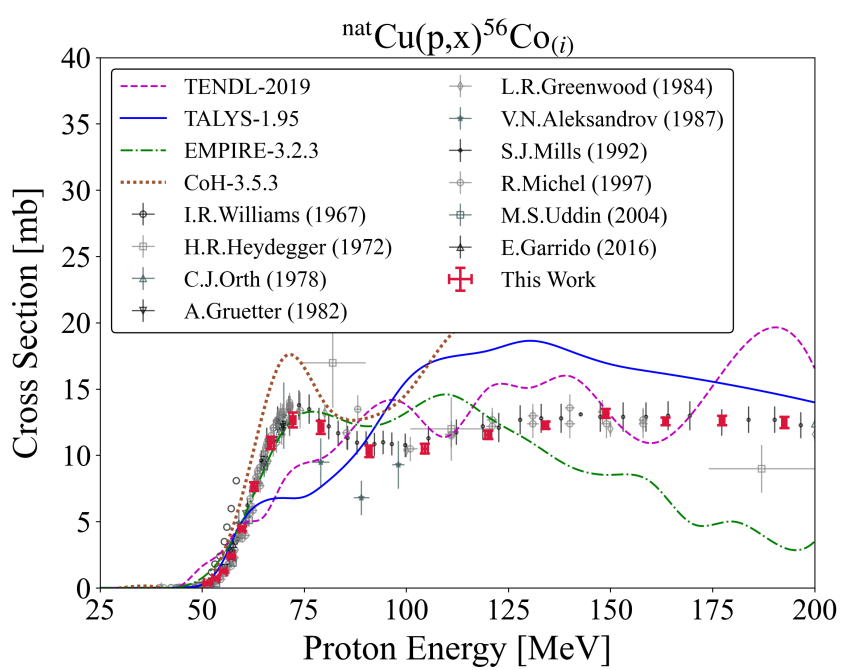

FIG. 40. Experimental and theoretical cross sections for ${ }^{56} \mathrm{Co}$ production.

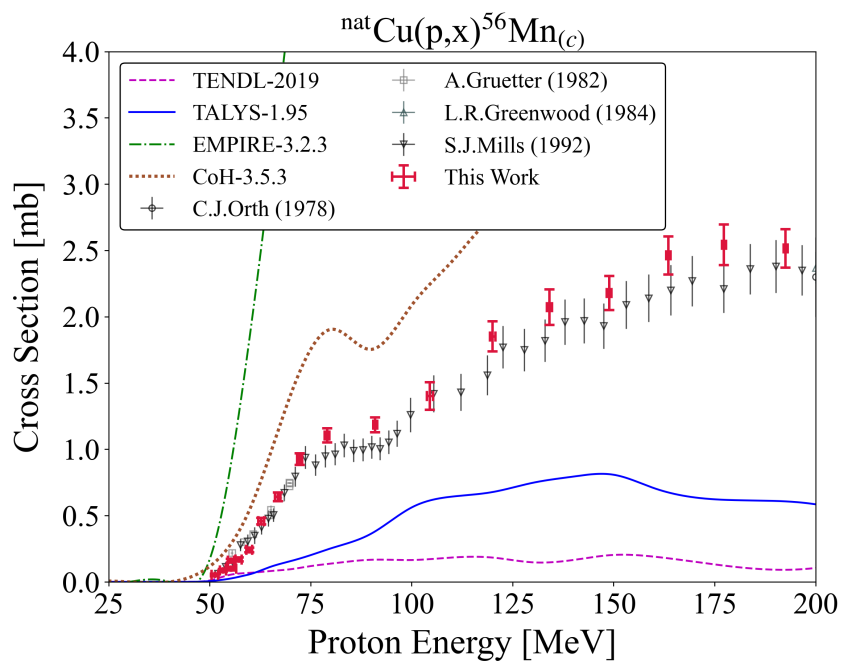

FIG. 41. Experimental and theoretical cross sections for ${ }^{56} \mathrm{Mn}$ production.

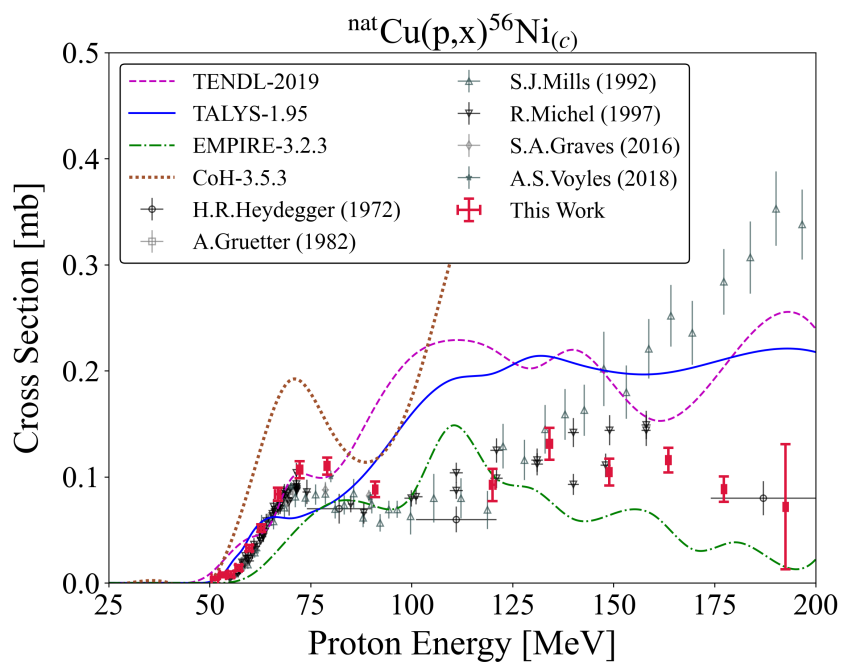

FIG. 42. Experimental and theoretical cross sections for ${ }^{56} \mathrm{Ni}$ production.

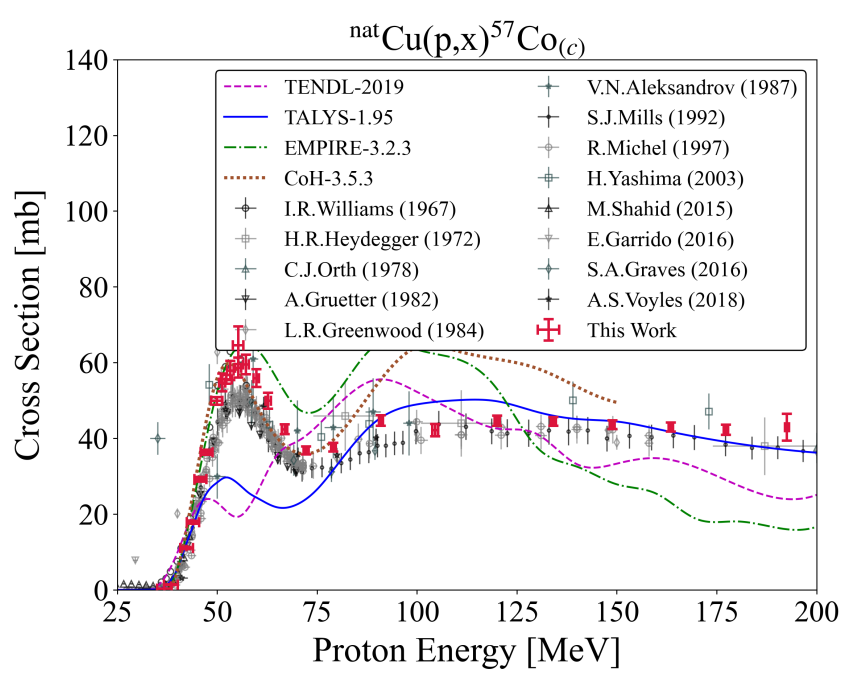

FIG. 43. Experimental and theoretical cross sections for ${ }^{57} \mathrm{Co}$ production.

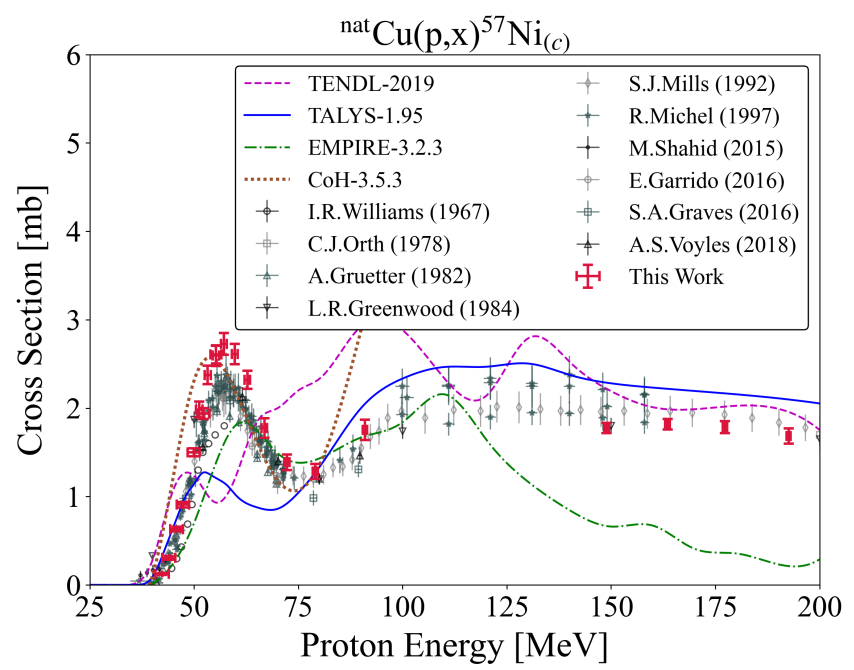

FIG. 44. Experimental and theoretical cross sections for ${ }^{57} \mathrm{Ni}$ production.

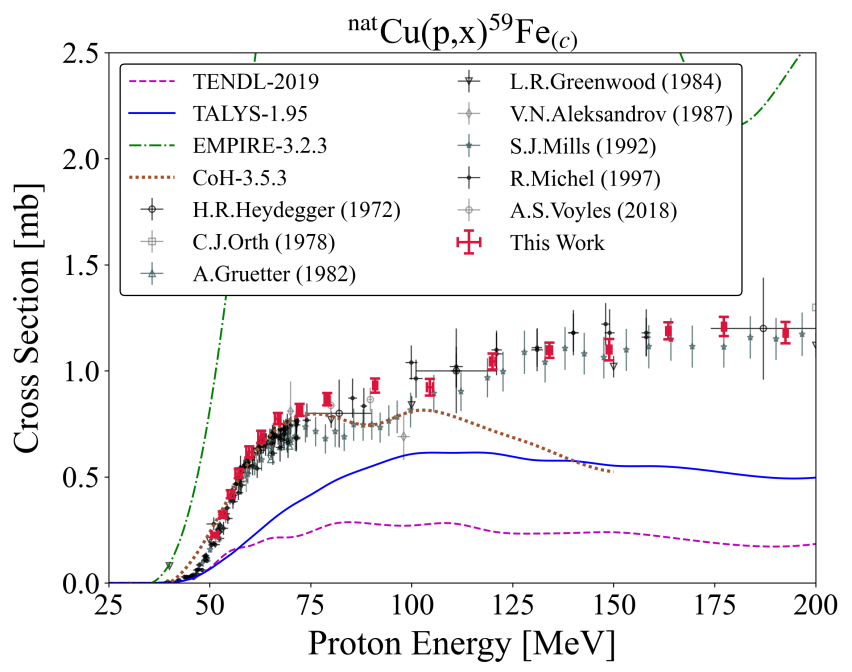

FIG. 45. Experimental and theoretical cross sections for ${ }^{59} \mathrm{Fe}$ production. 


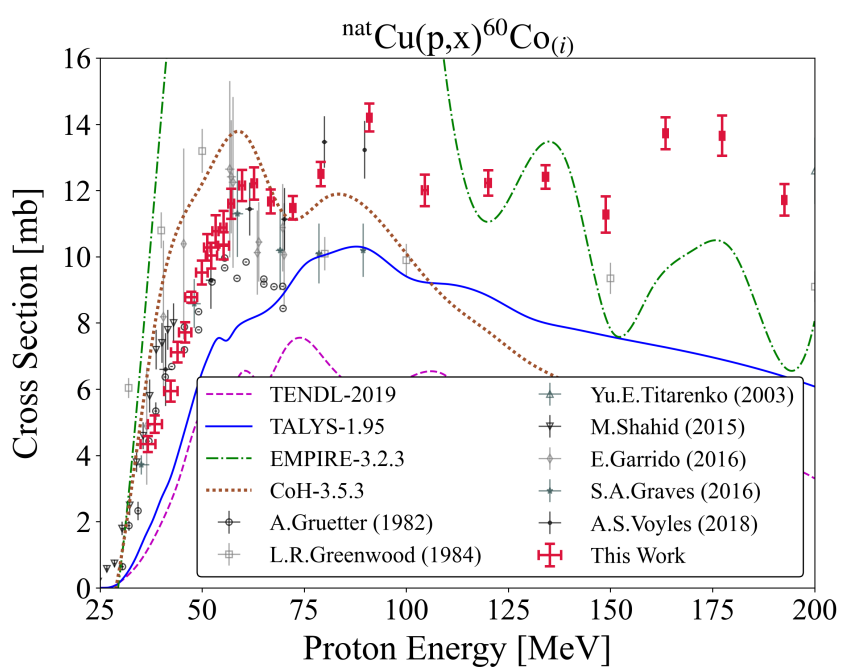

FIG. 46. Experimental and theoretical cross sections for ${ }^{60} \mathrm{Co}$ production.

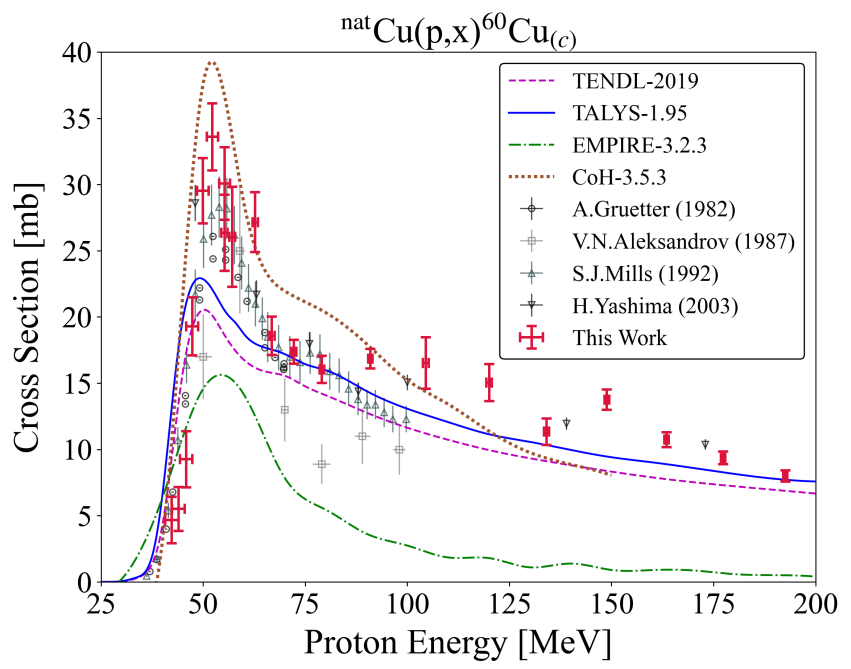

FIG. 47. Experimental and theoretical cross sections for ${ }^{60} \mathrm{Cu}$ production.

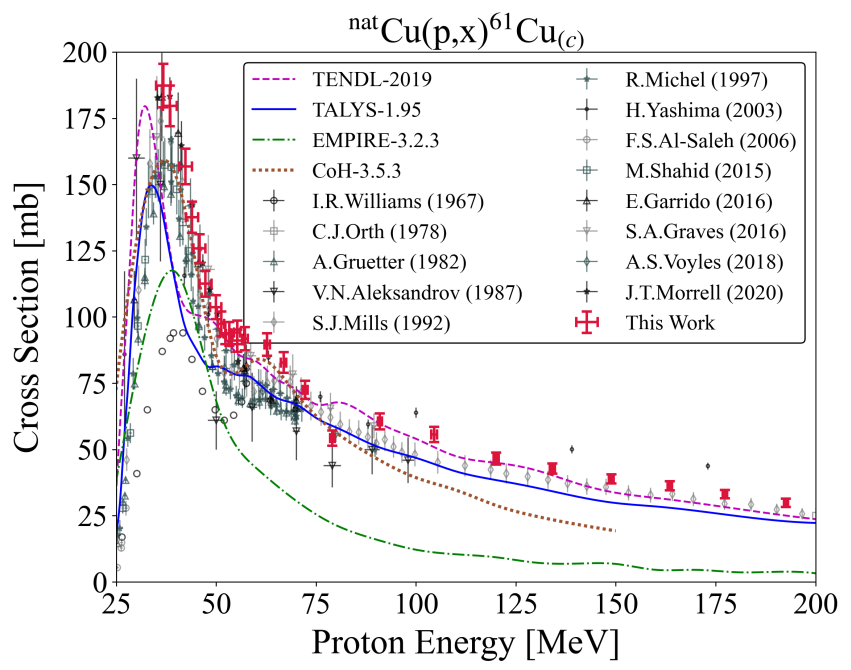

FIG. 48. Experimental and theoretical cross sections for ${ }^{61} \mathrm{Cu}$ production.

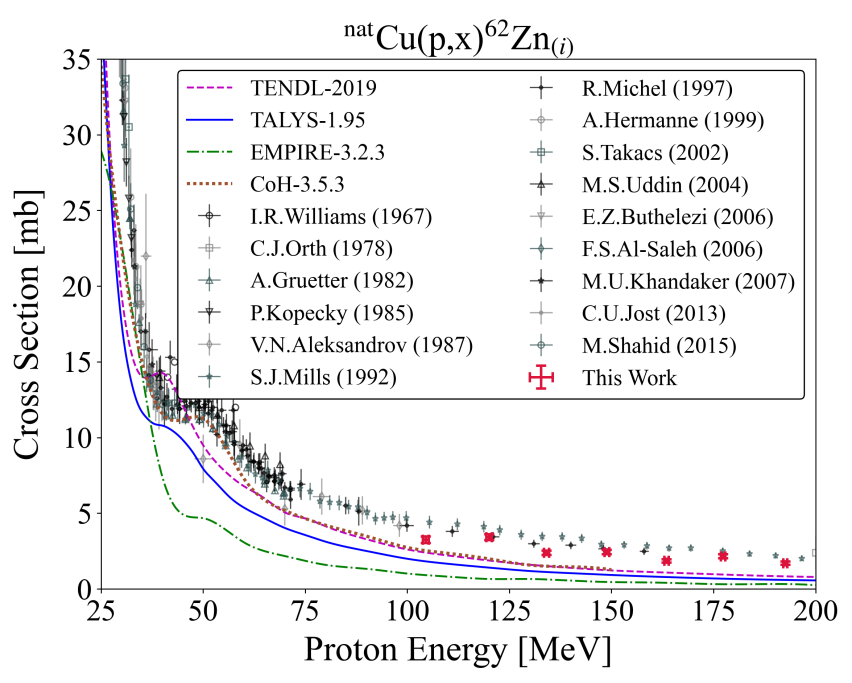

FIG. 49. Experimental and theoretical cross sections for ${ }^{62} \mathrm{Zn}$ production.

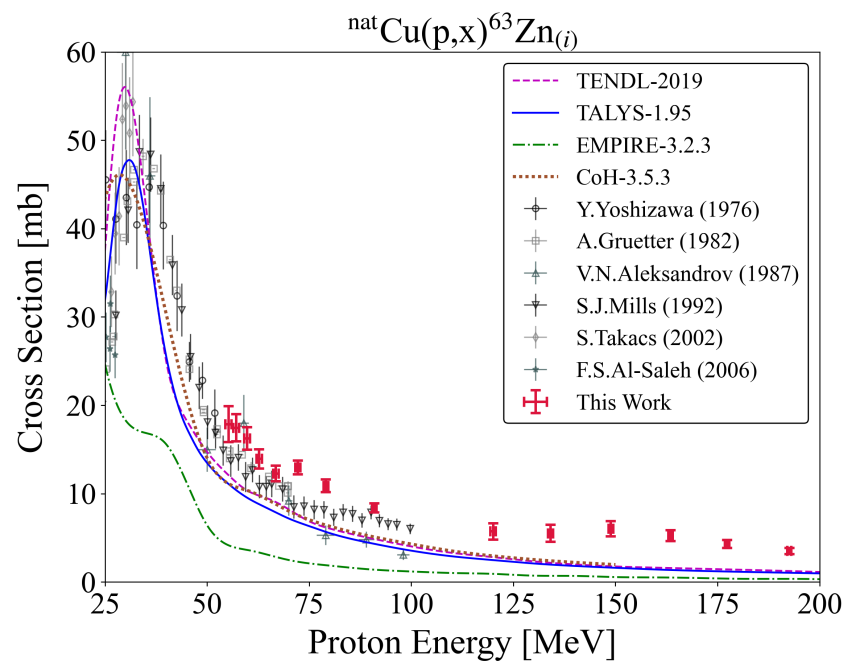

FIG. 50. Experimental and theoretical cross sections for ${ }^{63} \mathrm{Zn}$ production.

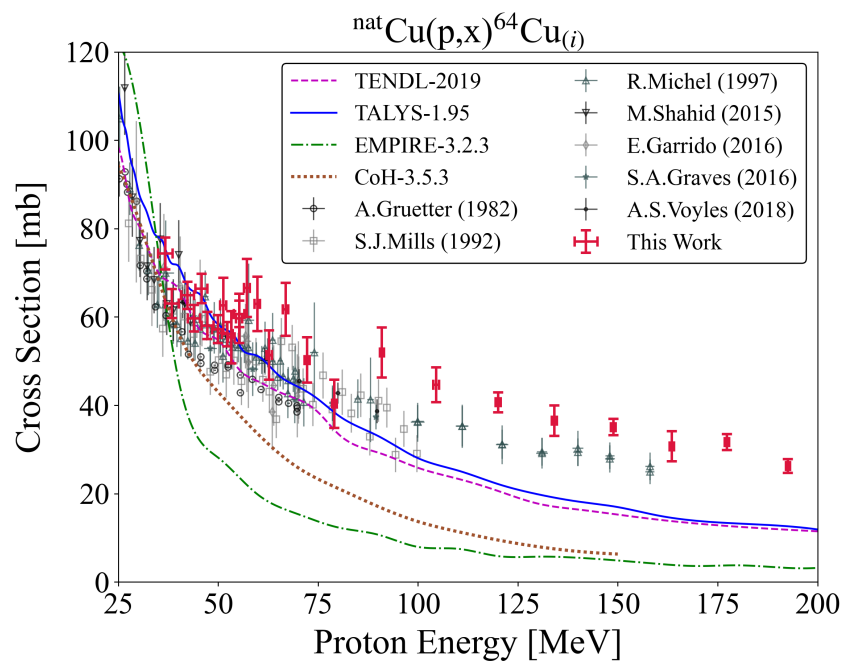

FIG. 51. Experimental and theoretical cross sections for ${ }^{64} \mathrm{Cu}$ production. 


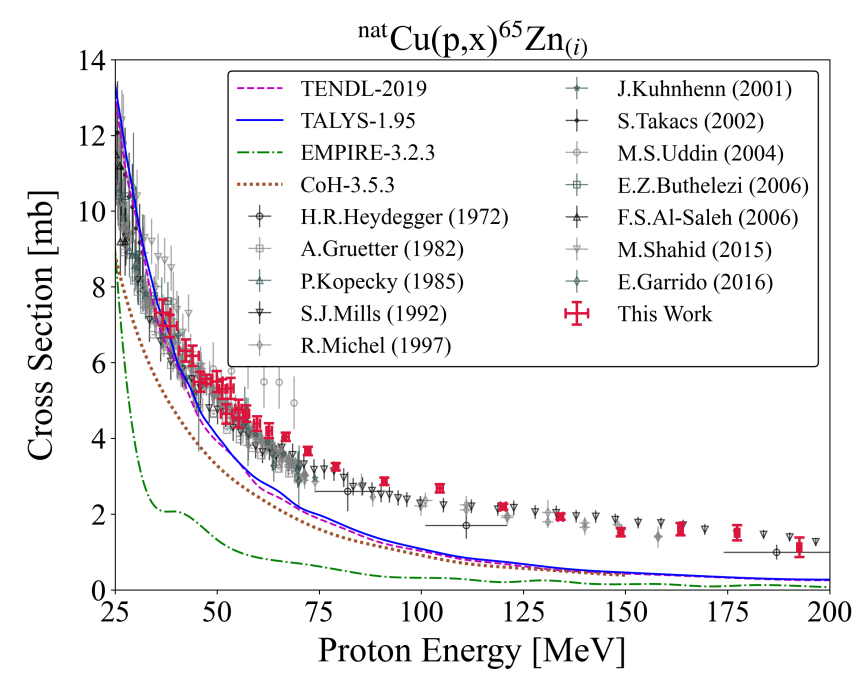

FIG. 52. Experimental and theoretical cross sections for ${ }^{65} \mathrm{Zn}$ production.

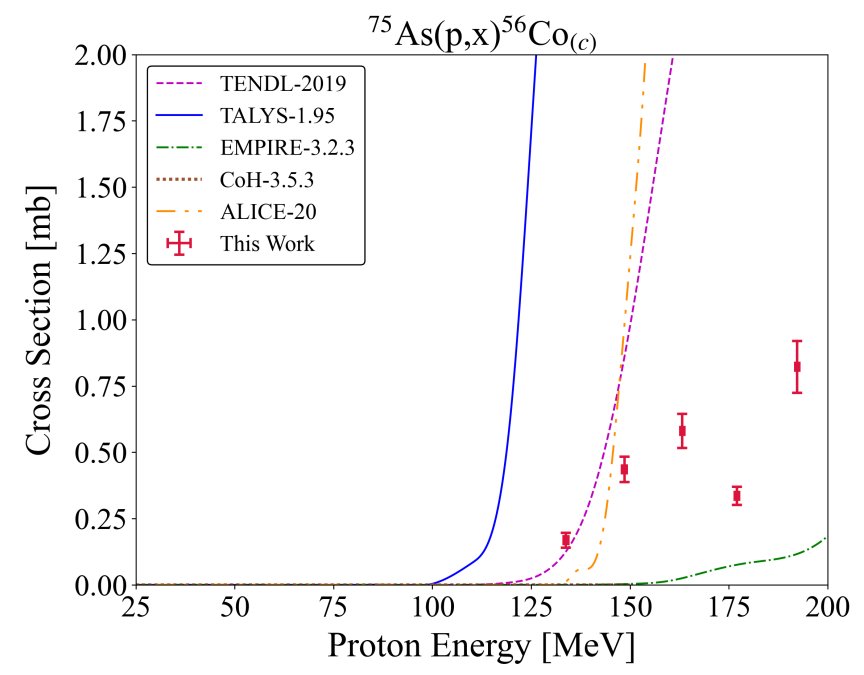

FIG. 53. Experimental and theoretical cross sections for ${ }^{56} \mathrm{Co}$ production.

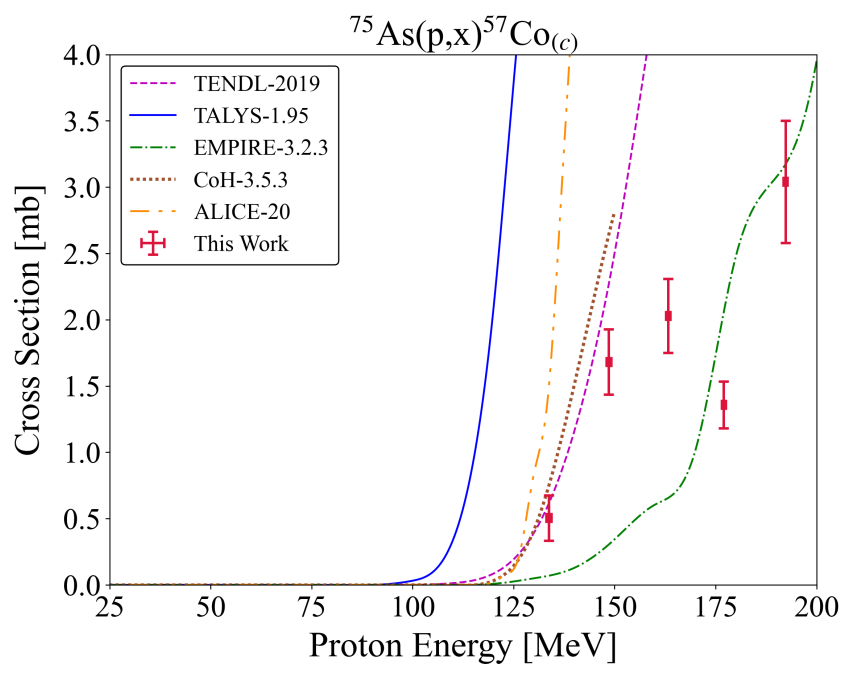

FIG. 54. Experimental and theoretical cross sections for ${ }^{57} \mathrm{Co}$ production.

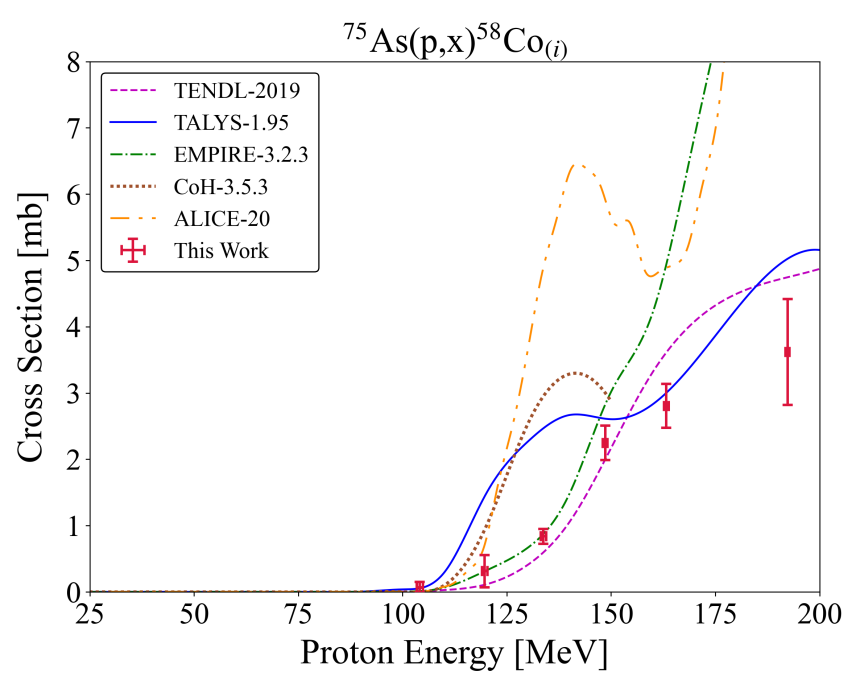

FIG. 55. Experimental and theoretical cross sections for ${ }^{58} \mathrm{Co}$ production.

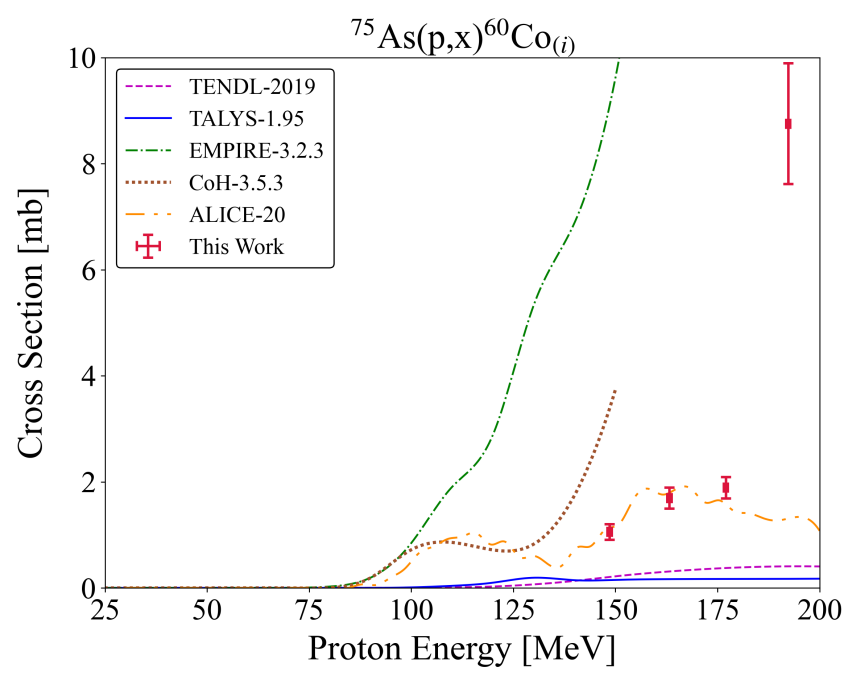

FIG. 56. Experimental and theoretical cross sections for ${ }^{60} \mathrm{Co}$ production.

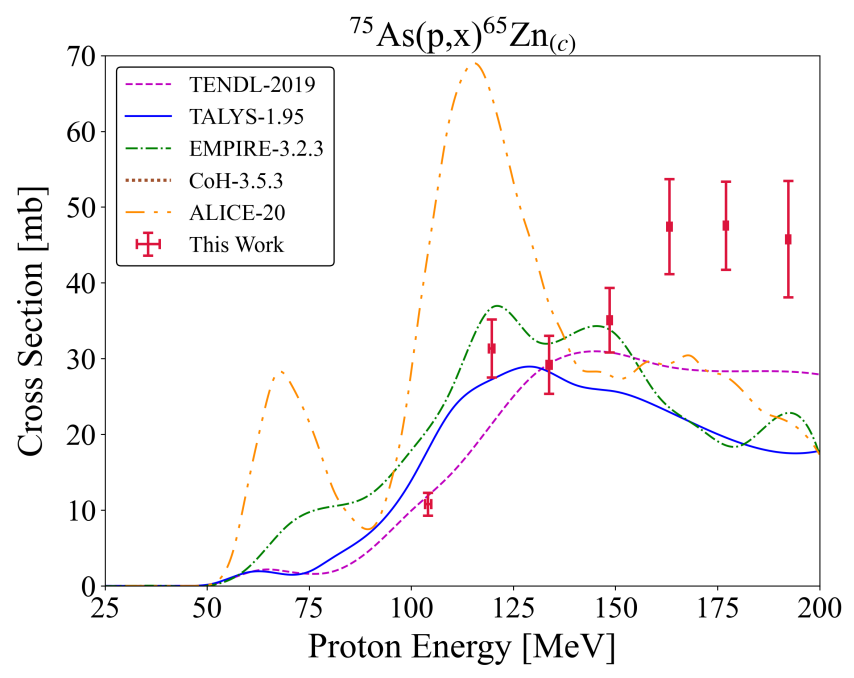

FIG. 57. Experimental and theoretical cross sections for ${ }^{65} \mathrm{Zn}$ production. 


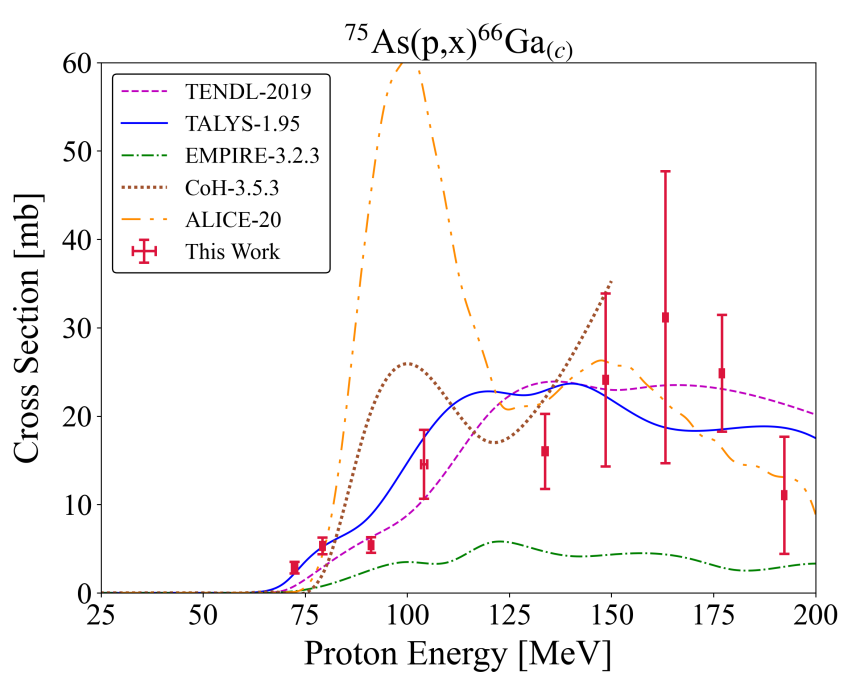

FIG. 58. Experimental and theoretical cross sections for ${ }^{66} \mathrm{Ga}$ production.

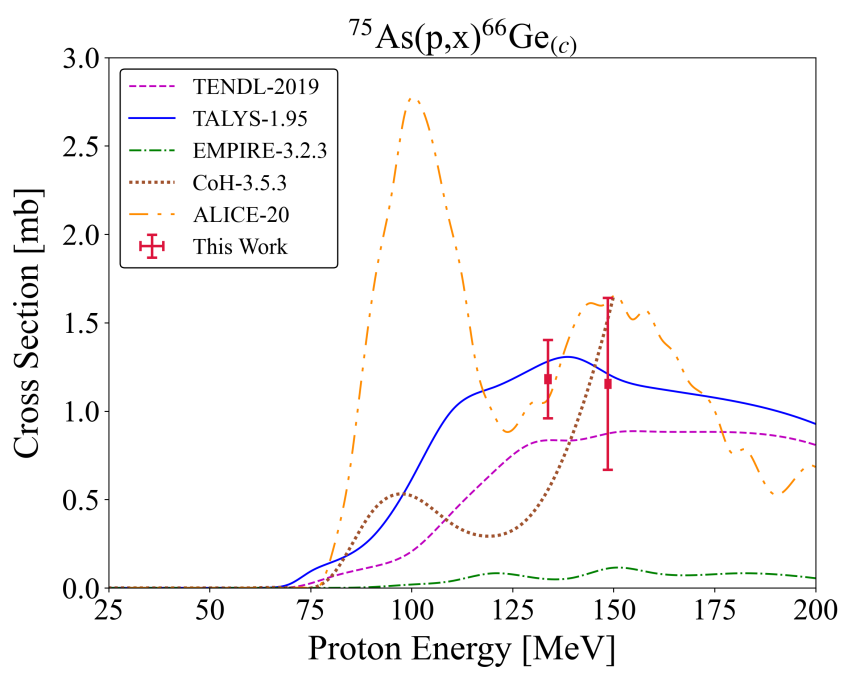

FIG. 59. Experimental and theoretical cross sections for ${ }^{66} \mathrm{Ge}$ production.

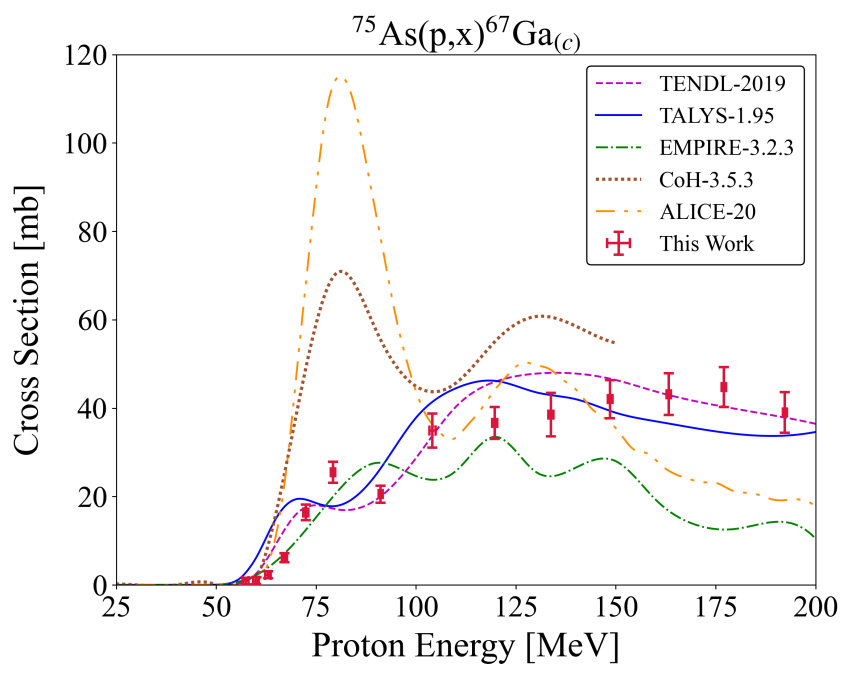

FIG. 60. Experimental and theoretical cross sections for ${ }^{67} \mathrm{Ga}$ production.

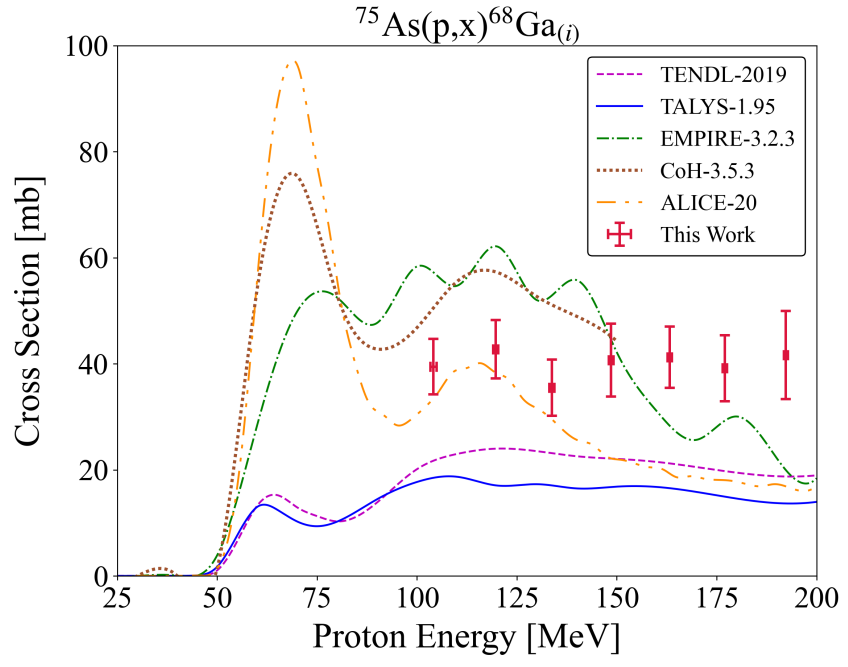

FIG. 61. Experimental and theoretical cross sections for ${ }^{68} \mathrm{Ga}$ production.

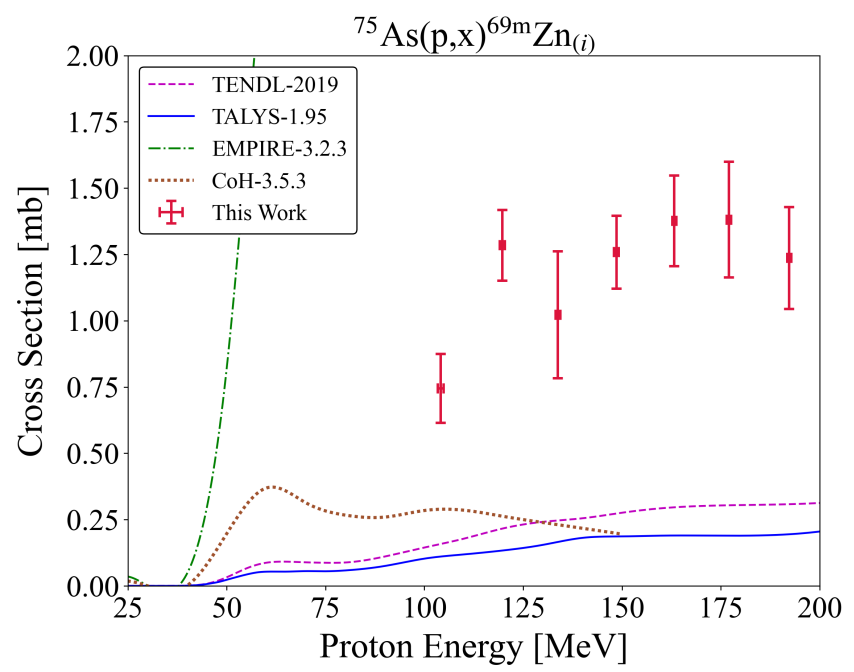

FIG. 62. Experimental and theoretical cross sections for ${ }^{69 \mathrm{~m}} \mathrm{Zn}$ production.

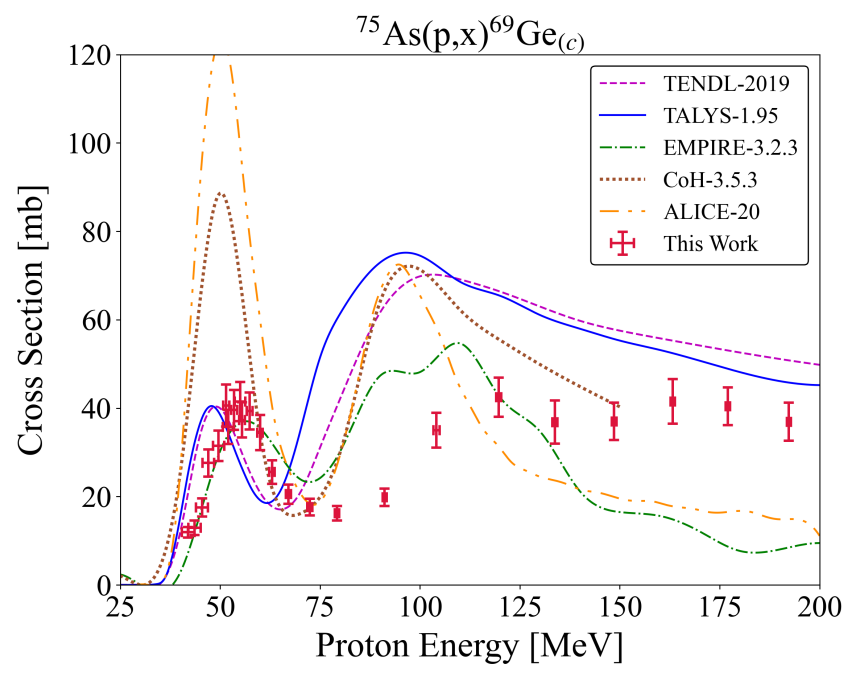

FIG. 63. Experimental and theoretical cross sections for ${ }^{69} \mathrm{Ge}$ production. 


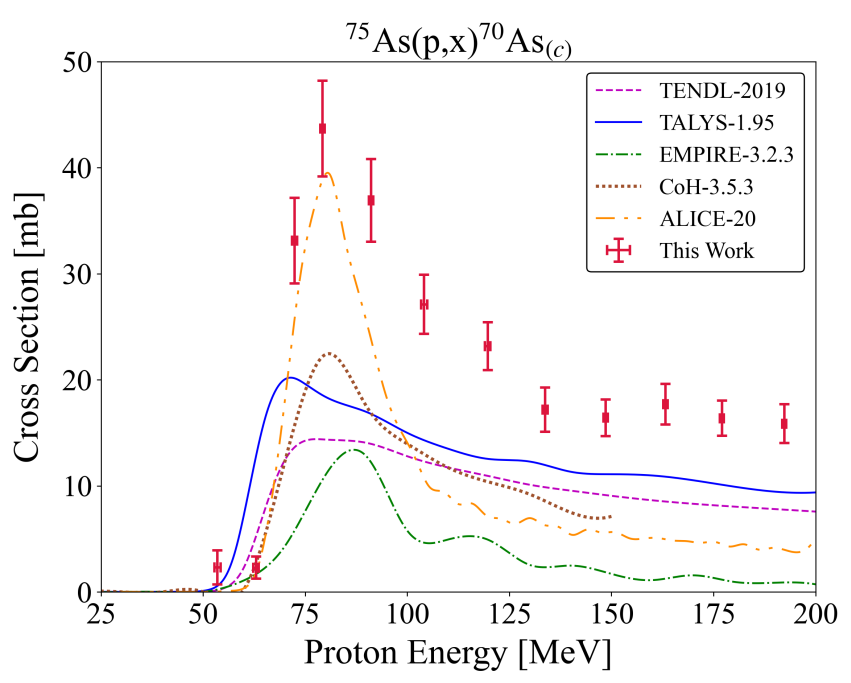

FIG. 64. Experimental and theoretical cross sections for ${ }^{70} \mathrm{As}$ production.

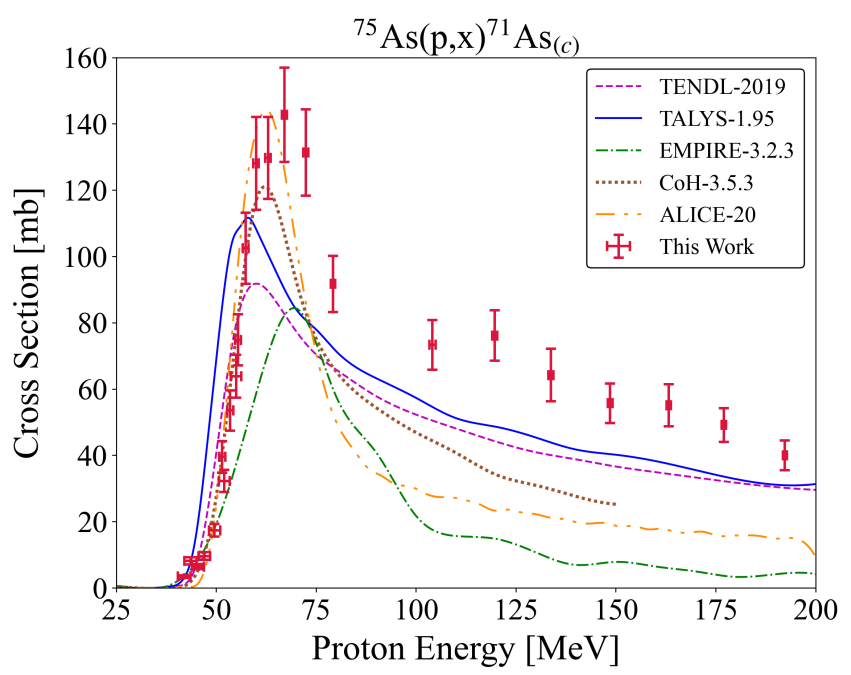

FIG. 65. Experimental and theoretical cross sections for ${ }^{71} \mathrm{As}$ production.

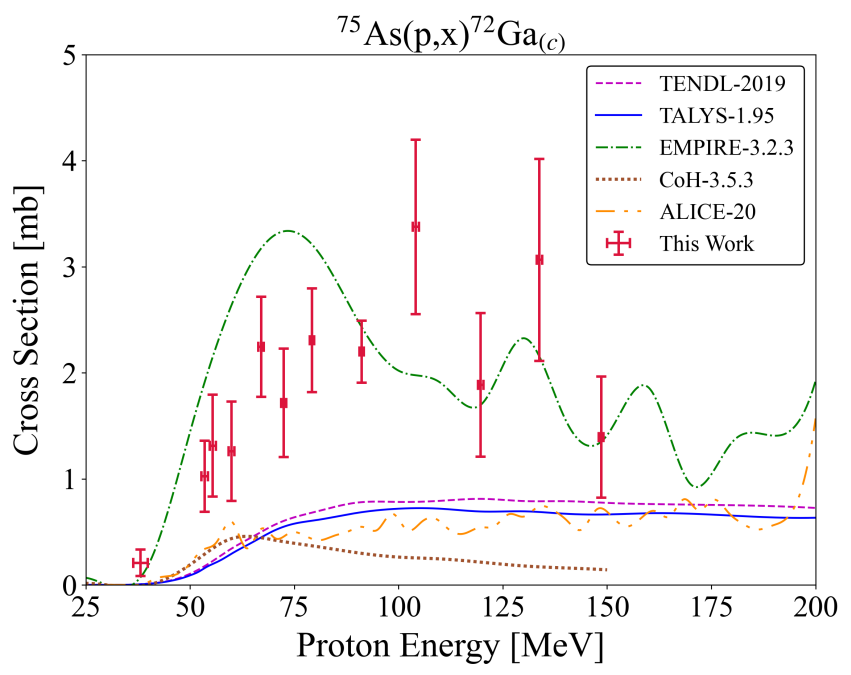

FIG. 66. Experimental and theoretical cross sections for ${ }^{72} \mathrm{Ga}$ production.

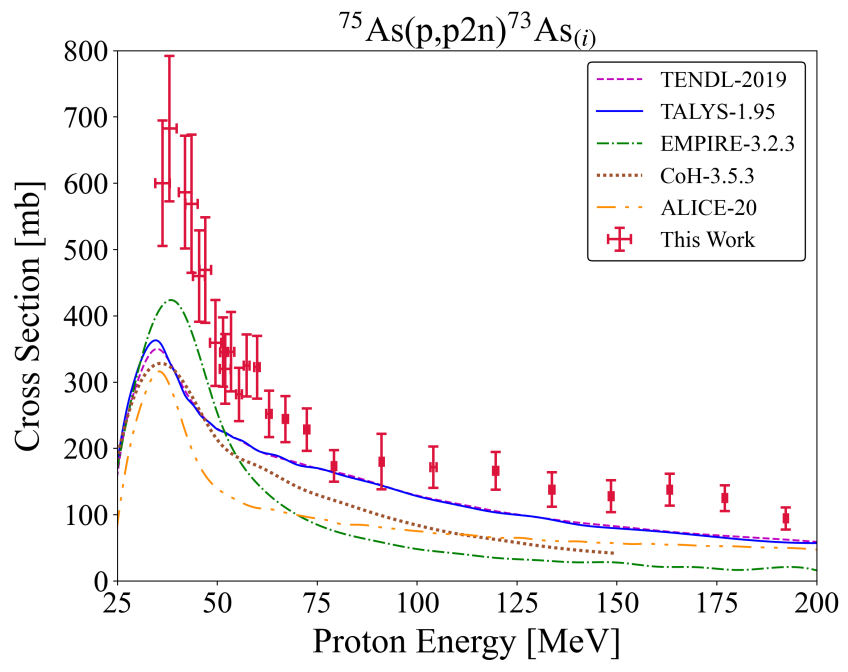

FIG. 67. Experimental and theoretical cross sections for ${ }^{73} \mathrm{As}$ production.

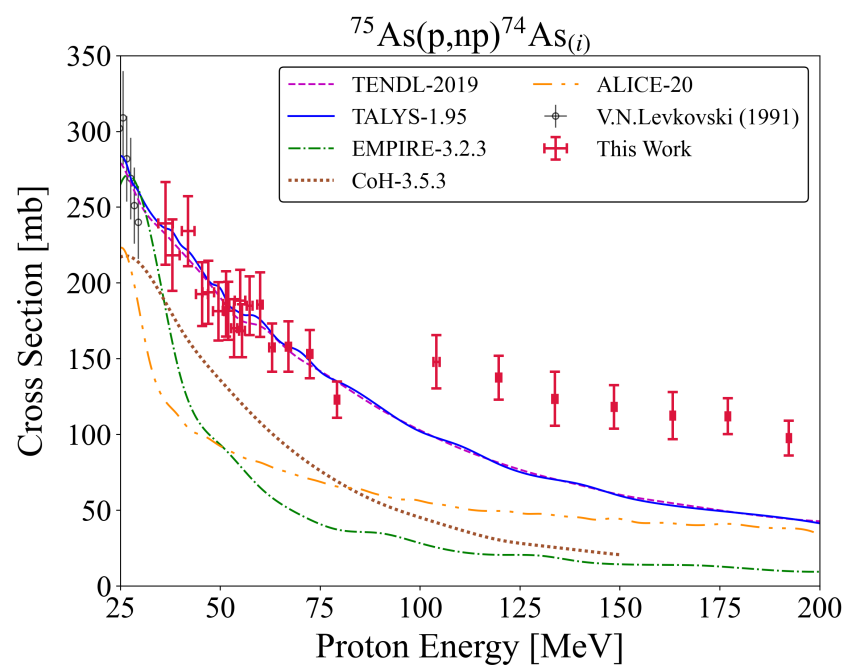

FIG. 68. Experimental and theoretical cross sections for ${ }^{74} \mathrm{As}$ production.

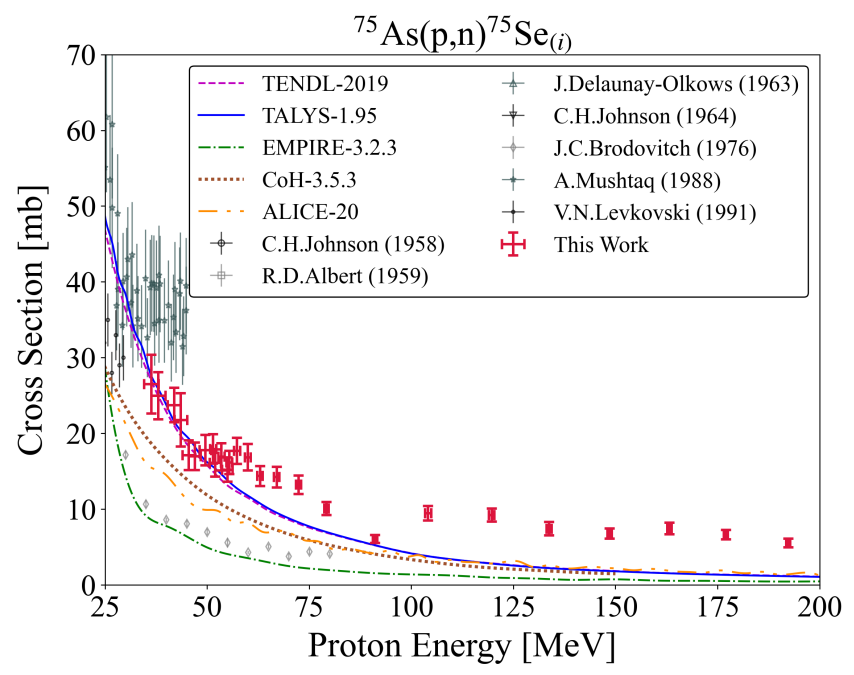

FIG. 69. Experimental and theoretical cross sections for ${ }^{75} \mathrm{Se}$ production. 


\section{Appendix D: TALYS Parameter Adjustments From Fitting Procedure}

The derived parameter adjustments from the fitting procedure applied to the ${ }^{75} \mathrm{As}(\mathrm{p}, \mathrm{x})$ data are listed in Table IX.

TABLE IX. ${ }^{75} \mathrm{As}(\mathrm{p}, \mathrm{x})$ best fit parameter adjustments derived from Fox et al. [21] procedure. The equidistant keyword adjusts the width of excitation energy binning and will be a default in updated TALYS versions. The strength keyword selects the gamma-ray strength model and has only a small impact in this charged-particle investigation. strength 8 performed comparably or slightly better than the other available models in TALYS.

\begin{tabular}{|c|c|}
\hline Parameter & Value \\
\hline ldmodel & $\begin{array}{l}6 \\
4{ }^{76-72} \mathrm{Se},{ }^{68} \mathrm{As} \\
5^{69} \mathrm{Ga}\end{array}$ \\
\hline strength & 8 \\
\hline equidistant & $\mathrm{y}$ \\
\hline M2constant & 0.80 \\
\hline M2limit & 3.9 \\
\hline M2shift & 0.55 \\
\hline $\mathrm{Kph}$ & 15.16 \\
\hline d1adjust $\mathrm{p}$ & 1.55 \\
\hline d1adjust n & 1.75 \\
\hline w1adjust p & 1.21 \\
\hline alphaomp & 6 \\
\hline deuteronomp & 4 \\
\hline Cstrip & $\begin{array}{ll}\text { a } & 0.85 \\
\text { d } & 2.4 \\
\text { h } & 0.55 \\
\text { t } & 0.55\end{array}$ \\
\hline Cknock & $\begin{array}{ll}\text { a } & 0.85 \\
\text { d } & 2.4 \\
\text { h } & 0.55 \\
\text { t } & 0.55\end{array}$ \\
\hline ctable & $\begin{array}{lll}34 & 73 & 0.24 \\
33 & 74 & 0.3 \\
33 & 73 & 0.75 \\
33 & 71 & -0.4 \\
32 & 69 & 0.285 \\
31 & 67 & -0.45\end{array}$ \\
\hline ptable & $\begin{array}{lll}34 & 73 & -0.65 \\
34 & 72 & 0.14 \\
33 & 73 & -1.85 \\
32 & 69 & -0.25 \\
31 & 67 & 5.5\end{array}$ \\
\hline
\end{tabular}


[1] D. Abriola and A. A. Sonzogni, Nuclear Data Sheets for $A=72$, Nuclear Data Sheets 111, 1 (2010).

[2] M. Jennewein, S. M. Qaim, P. V. Kulkarni, R. P. Mason, A. Hermanne, and F. Rösch, A no-carrier-added ${ }^{72} \mathrm{Se} /{ }^{72} \mathrm{As}$ radionuclide generator based on solid phase extraction, Radiochimica Acta 93, 579 (2005).

[3] F. T. Tárkányi, A. V. Ignatyuk, A. Hermanne, R. Capote, B. V. Carlson, J. W. Engle, M. A. Kellet, T. Kibedi, G. N. Kim, F. G. Kondev, M. Hussain, O. Lebeda, A. Luca, Y. Nagai, H. Naik, A. L. Nichols, F. M. Nortier, S. V. Suryanarayana, S. Takacs, and M. Verpelli, Recommended nuclear data for medical radioisotope production: diagnostic positron emitters, Journal of Radioanalytical and Nuclear Chemistry 319, 533 (2019).

[4] J. Katakura and Z. D. Wu, Nuclear Data Sheets for A = 124, Nuclear Data Sheets 109, 1655 (2008).

[5] G. L. Cascini, A. N. Asabella, A. Notaristefano, A. Restuccia, C. Ferrari, D. Rubini, C. Altini, and G. Rubini, ${ }^{124}$ Iodine: A Longer-Life Positron Emitter Isotope - New Opportunities in Molecular Imaging, BioMed Research International 2014, 1 (2014).

[6] B. Singh and N. Nica, Nuclear Data Sheets for A = 77, Nuclear Data Sheets 113, 1115 (2012).

[7] A. J. DeGraffenreid, D. G. Medvedev, T. E. Phelps, M. D. Gott, S. V. Smith, S. S. Jurisson, and C. S. Cutler, Cross-section measurements and production of Se with medium to high energy protons using arsenic containing targets, Radiochimica Acta 107, 279 (2019).

[8] M. Jennewein, M. A. Lewis, D. Zhao, E. Tsyganov, N. Slavine, J. He, L. Watkins, V. D. Kodibagkar, S. O. Kelly, P. Kulkarni, P. P. Antich, A. Hermanne, F. Ro, R. P. Mason, and P. E. Thorpe, Vascular Imaging of Solid Tumors in Rats with a Radioactive ArsenicLabeled Antibody that Binds Exposed Phosphatidylserine, Clinical Cancer Research 14, 1377 (2008).

[9] V. A. Sanders and C. S. Cutler, Radioarsenic: A promising theragnostic candidate for nuclear medicine, Nuclear Medicine and Biology 92, 184 (2021).

[10] P. A. Ellison, T. E. Barnhart, F. Chen, H. Hong, Y. Zhang, C. P. Theuer, W. Cai, R. J. Nickles, and O. T. DeJesus, High Yield Production and Radiochemical Isolation of Isotopically Pure Arsenic-72 and Novel Radioarsenic Labeling Strategies for the Development of Theranostic Radiopharmaceuticals, Bioconjugate Chemistry 27, 179 (2017).

[11] B. Ballard, F. M. Nortier, E. R. Birnbaum, K. D. John, D. R. Phillips, and M. Fassbender, Radioarsenic from a Portable ${ }^{72} \mathrm{Se} /{ }^{72}$ As Generator: A Current Perspective, Current Radiopharmaceuticals 5, 264 (2012).

[12] B. Ballard, D. Wycoff, E. R. Birnbaum, K. D. John, J. W. Lenz, S. S. Jurisson, C. S. Cutler, F. M. Nortier, W. A. Taylor, and M. E. Fassbender, Selenium-72 formation via ${ }^{\text {nat }} \operatorname{Br}(\mathrm{p}, \mathrm{x})$ induced by $100 \mathrm{MeV}$ Protons: Steps towards a novel ${ }^{72} \mathrm{Se} /{ }^{72}$ As generator system, Applied Radiation and Isotopes 70, 595 (2012).

[13] M. Fassbender, D. De Villiers, M. Nortier, and N. Van der Walt, The ${ }^{n a t} \operatorname{Br}(\mathrm{p}, \mathrm{x})^{73,75}$ Se nuclear processes: a convenient route for the production of radioselenium tracers relevant to amino acid labelling, Applied Radiation and Isotopes 54, 905 (2001).
[14] S. Takács, M. P. Takács, F. Ditrói, M. Aikawa, H. Haba, and Y. Komori, Activation cross sections of longer-lived radionuclides produced in germanium by alpha particle irradiation, Nuclear Instruments and Methods in Physics Research B 383, 213 (2016).

[15] E. A. McCutchan, Nuclear Data Sheets for $\mathrm{A}=68$, Nuclear Data Sheets 113, 1735 (2012).

[16] L. Martiniova, L. D. Palatis, E. Etchebehere, and G. Ravizzini, Gallium-68 in Medical Imaging, Current Radiopharmaceuticals 9, 187 (2016).

[17] F. Roesch and D. V. Filosofov, Production of Long Lived Parent Radionuclides for Generators : ${ }^{68} \mathrm{Ge},{ }^{82} \mathrm{Sr}$, ${ }^{90} \mathrm{Sr}$ and ${ }^{188} \mathrm{~W}$, IAEA Radioisotopes and Radiopharmaceuticals Series 2 (2010).

[18] B. P. Burke and S. J. Archibald, Handbook of Radiopharmaceuticals: Methodology and Applications, 2nd ed., edited by P. Scott and M. Kilbourn (2020) pp. 291323.

[19] C. Beard, D. L. Clark, G. D. Jarvinen, A. Migliori, W. Runde, M. Coonley, K. Parker, E. Lorusso, and L. Archuleta, Accelerator Radioisotopes Save Lives: Part II, Actinide Research Quarterly (LALP-10-011), 1 (2010).

[20] C. Kratochwil, F. Bruchertseifer, H. Rathke, M. Hohenfellner, F. L. Giesel, U. Haberkorn, and A. Morgenstern, Targeted $\alpha$-Therapy of Metastatic Castration-Resistant Prostate Cancer with ${ }^{225}$ Ac-PSMA-617: Swimmer-Plot Analysis Suggests Efficacy Regarding Duration of Tumor Control, Journal of Nuclear Medicine 59, 795 (2018).

[21] M. B. Fox, A. S. Voyles, J. T. Morrell, L. A. Bernstein, A. M. Lewis, A. J. Koning, J. C. Batchelder, E. R. Birnbaum, C. S. Cutler, D. G. Medvedev, F. M. Nortier, E. M. O'Brien, and C. Vermeulen, Investigating high-energy proton-induced reactions on spherical nuclei: Implications for the preequilibrium exciton model, Physical Review C 103, 034601 (2021).

[22] S. Hogle, E. O'Brien, A. S. Voyles, K. Kolos, and V. Sobes, Predictive Codes for Isotope Production, in Workshop for Applied Nuclear Data Activities (WANDA 2021) (Washington, D.C., 2021).

[23] A. S. Voyles, L. A. Bernstein, E. R. Birnbaum, J. W. Engle, S. A. Graves, T. Kawano, A. M. Lewis, and F. M. Nortier, Excitation functions for $(\mathrm{p}, \mathrm{x})$ reactions of niobium in the energy range of $\mathrm{E}_{p}=40-90 \mathrm{MeV}$, Nuclear Instruments and Methods in Physics Research B 429, 53 (2018).

[24] J. T. Morrell, A. S. Voyles, M. S. Basunia, J. C. Batchelder, E. F. Matthews, and L. A. Bernstein, Measurement of ${ }^{139} \mathrm{La}(\mathrm{p}, \mathrm{x})$ cross sections from $35-60 \mathrm{MeV}$ by stacked-target activation, The European Physical Journal A 56, 13 (2020).

[25] S. A. Graves, P. A. Ellison, T. E. Barnhart, H. F. Valdovinos, R. Eva, F. M. Nortier, R. J. Nickles, and J. W. Engle, Nuclear excitation functions of proton-induced reactions $\left(\mathrm{E}_{p}=35-90 \mathrm{MeV}\right)$ from $\mathrm{Fe}, \mathrm{Cu}, \mathrm{Al}$, Nuclear Instruments and Methods in Physics Research B 386, 44 (2016).

[26] J. W. Engle, S. G. Mashnik, J. W. Weidner, L. E. Wolfsberg, M. Fassbender, K. Jackman, A. Couture, L. J. Bitteker, J. L. Ullmann, M. S. Gulley, C. Pillai, K. D. 
John, E. R. Birnbaum, and F. M. Nortier, Cross sections from proton irradiation of thorium at $800 \mathrm{MeV}$, Physical Review C 88, 014604 (2013).

[27] J. W. Engle, S. G. Mashnik, L. A. Parker, K. R. Jackman, L. J. Bitteker, J. L. Ullmann, M. S. Gulley, C. Pillai, K. D. John, E. R. Birnbaum, and F. M. Nortier, Nuclear excitation functions from 40 to $200 \mathrm{MeV}$ proton irradiation of terbium, Nuclear Instruments and Methods in Physics Research B 366, 206 (2016).

[28] A. S. Voyles, M. B. Fox, J. T. Morrell, M. P. Zach, E. K. Still, L. A. Bernstein, W. D. Frey, and B. J. Mehciz, Preparation and Characterization of Thin Arsenic Targets for Stacked-Target Experiments, arXiv (2021), arXiv:2106.05524.

[29] W. J. Gallagher and S. J. Cipolla, A Model-Based Efficiency Calibration of a $\mathrm{Si}(\mathrm{Li})$ Detector in the Energy Region From 3 to $140 \mathrm{keV}$, Nuclear Instruments and Methods 122, 405 (1974).

[30] J. T. Morrell, Curie: Python Toolkit for Experimental Nuclear Data (2020).

[31] J. T. Morrell, NPAT: Nuclear physics analysis tools (2019).

[32] A. Hermanne, A. V. Ignatyuk, R. Capote, B. V. Carlson, J. W. Engle, M. A. Kellett, and T. Kib, Reference Cross Sections for Charged-particle Monitor Reactions, Nuclear Data Sheets 148, 338 (2018).

[33] A. S. Voyles, A. M. Lewis, J. T. Morrell, M. Shamsuzzoha Basunia, L. A. Bernstein, J. W. Engle, S. A. Graves, and E. F. Matthews, Proton-induced reactions on $\mathrm{Fe}, \mathrm{Cu}, \& \mathrm{Ti}$ from threshold to $55 \mathrm{MeV}$, European Physics Journal A 57, 1 (2021).

[34] A. J. Koning, S. Hilaire, and S. Goriely, TALYS-1.95 A Nuclear Reaction Program (2019).

[35] T. Kawano, Unified Coupled-Channels and HauserFeshbach Model Calculation for Nuclear Data Evaluation (LA-UR 19-20315), Tech. Rep. (2019) arXiv:1901.05641.

[36] M. Herman, R. Capote, B. V. Carlson, P. Oblozinsky, M. Sin, A. Trkov, H. Wienke, and V. Zerkin, EMPIRE: Nuclear Reaction Model Code System for Data Evaluation, Nuclear Data Sheets 108, 2655 (2007).

[37] M. Blann and J. Bisplinghoff, ALICE/LIVERMORE Precompound Compound Decay, Tech. Rep. (Lawrence Livermore National Laboratory, 1982).

[38] A. J. Koning and D. Rochman, Modern Nuclear Data Evaluation with the TALYS Code System, Nuclear Data Sheets 113, 2841 (2012).

[39] A. Mushtaq, S. M. Qaim, and G. Stöcklin, Production of ${ }^{73} \mathrm{Se}$ via $(\mathrm{p}, 3 \mathrm{n})$ and $(\mathrm{d}, 4 \mathrm{n})$ Reactions on Arsenic, International Journal of Radiation Applications and Instrumentation Part A 39, 1085 (1988).

[40] S. M. Qaim, A. Mushtaq, and M. Uhl, Isomeric crosssection ratio for the formation of ${ }^{73 m, g}$ Se in various nuclear processes, Physical Review C 38, 645 (1988).

[41] J. C. Brodovitch, J. J. Hogan, and K. Burns, The Preequilibrium Statistical Model: Comparison of Calculations With Two (p,xn) Reactions, Journal of Inorganic and Nuclear Chemistry 38, 1581 (1976).

[42] V. N. Levkovski, Middle Mass Nuclides $(A=40-100)$ Activation Cross Sections by Medium Energy ( $E=10-50$ $\mathrm{MeV}$ ) Protons and $\alpha$-particles (Experiments and Systematics) (Inter-Vesi, Moscow, 1991).

[43] B. L. Cohen and E. G. Newman, (p,pn) and (p,2n) Cross Sections in Medium Weight Elements, Physical Review
99, 718 (1953).

[44] S. Neumann, Activation experiments with mediumenergy neutrons and the production of extraterrestrial matter, Ph.D. thesis, University of Hanover, Germany (1999).

[45] E. Garrido, C. Duchemin, A. Guertin, F. Haddad, N. Michel, and V. Métivier, New excitation functions for proton induced reactions on natural titanium, nickel and copper up to $70 \mathrm{MeV}$, Nuclear Instruments and Methods in Physics Research B 383, 191 (2016).

[46] R. L. Brodzinski, L. A. Rancitelli, J. A. Cooper, and N. A. Wogman, High-Energy Proton Spallation of Titanium, Physical Review C 4, 1250 (1971).

[47] R. Michel, F. Peiffer, and R. Stuck, Measurement and hybrid model analysis of integral excitation functions for proton-induced reactions on vanadium, manganese and cobalt up to $200 \mathrm{MeV}$, Nuclear Physics A 441, 617 (1985).

[48] D. Fink, J. Sisterson, S. Vogt, G. Herzog, A. Magliss, J. Klein, R. Middleton, A. Koehler, and A. Magliss, Production of ${ }^{41} \mathrm{Ca}$ and $\mathrm{K}, \mathrm{Sc}$ and $\mathrm{V}$ short-lived isotopes by the irradiation of Ti with 35 to $150 \mathrm{MeV}$ protons: applications to solar cosmic ray studies, Nuclear Instruments and Methods in Physics Research B 52, 601 (1990).

[49] R. Michel, G. Brinkmann, H. Weigel, and W. Herr, Proton-induced Reactions on Titanium with Energies Between 13 and $45 \mathrm{MeV}$, Journal of Inorganic and Nuclear Chemistry 40, 1845 (1978).

[50] R. Michel, R. Bodemann, H. Busemann, R. Daunke, M. Gloris, H. J. Lange, B. Klug, A. Krins, I. Leya, M. Lüpke, S. Neumann, H. Reinhardt, M. SchnatzBüttgen, U. Herpers, T. Schiekel, F. Sudbrock, B. Holmqvist, H. Condé, P. Malmborg, M. Suter, B. Dittrich-Hannen, P. W. Kubik, H. A. Synal, and D. Filges, Cross sections for the production of residual nuclides by low- and medium-energy protons from the target elements $\mathrm{C}, \mathrm{N}, \mathrm{O}, \mathrm{Mg}, \mathrm{Al}, \mathrm{Si}, \mathrm{Ca}, \mathrm{Ti}, \mathrm{V}, \mathrm{Mn}$, $\mathrm{Fe}, \mathrm{Co}, \mathrm{Ni}, \mathrm{Cu}, \mathrm{Sr}, \mathrm{Y}, \mathrm{Zr}, \mathrm{Nb}, \mathrm{Ba}$ and $\mathrm{Au}$, Nuclear Instruments and Methods in Physics Research B 129, 153 (1997).

[51] M. U. Khandaker, K. Kim, M. W. Lee, K. S. Kim, G. N. Kim, Y. S. Cho, and Y. O. Lee, Investigations of the ${ }^{n a t} \mathrm{Ti}(\mathrm{p}, \mathrm{x}){ }^{43,44 m, 44 g, 46,47,48} \mathrm{Sc},{ }^{48} \mathrm{~V}$ nuclear processes up to $40 \mathrm{MeV}$, Applied Radiation and Isotopes 67, 1348 (2009).

[52] K. Zarie, N. Al-Hammad, and A. Azzam, Experimental study of excitation functions of some proton induced reactions on ${ }^{n a t} \mathrm{Ti}$ for beam monitoring purposes, Radiochimica Acta 94, 795 (2006).

[53] J. Cervenak and O. Lebeda, New cross-section data for proton-induced reactions on ${ }^{n a t} \mathrm{Ti}$ and ${ }^{n a t} \mathrm{Cu}$ with special regard to the beam monitoring, Nuclear Instruments and Methods in Physics Research B 480, 78 (2020).

[54] P. Kopecky, F. Szelecsenyi, T. Molnar, P. Mikecz, and F. T. Tárkányi, Excitation Functions of $(p, x n)$ Reactions on ${ }^{n a t} \mathrm{Ti}$ : Monitoring of Bombarding Proton Beams, Applied Radiation and Isotopes 44, 687 (1993).

[55] F. Bringas, M. T. Yamashita, I. D. Goldman, P. R. Pascholati, and V. Sciani, Measurement of ProtonInduced Reaction Cross Sections in $\mathrm{Ti}, \mathrm{Ni}$ and $\mathrm{Zr}$ near the Threshold, AIP Conference Proceedings 769, 13 (2005). 
[56] A. Hermanne, F. T. Tárkányi, S. Takács, F. Ditrói, and N. Amjed, Excitation functions for production of ${ }^{46} \mathrm{Sc}$ by deuteron and proton beams in ${ }^{n a t} \mathrm{Ti}$ : A basis for additional monitor reactions, Nuclear Instruments and Methods in Physics Research B 338, 31 (2014).

[57] B. Dittrich, U. Herpers, and T. Schiffmann, Thin-Target excitation functions for proton-produced long-lived radionuclides, Tech. Rep. (Fed. Rep. Germ. Report to the I.N.D.C. No.32/LN+SPECIAL, 1988).

[58] F. T. Tárkányi, F. Szelecsenyi, and P. Kopecky, Qaim S.M. (Ed.) Nuclear Data for Science and Technology, edited by Springer (Springer, Berlin, Heidelberg, 1992) pp. 529-532.

[59] J. R. Walton, D. Heymann, A. Yaniv, D. Edgerley, and M. W. Rowe, Cross Sections for He and Ne Isotopes in Natural $\mathrm{Mg}, \mathrm{Al}$, and $\mathrm{Si}$, He Isotopes in $\mathrm{CaF}_{2}, \mathrm{Ar}$ Isotopes in Natural $\mathrm{Ca}$, and Radionuclides in Natural $\mathrm{Al}, \mathrm{Si}, \mathrm{Ti}, \mathrm{Cr}$, and Stainless Steel Induced by 12- to 45-MeV Protons, Journal of Geophysical Research 81, 5689 (1976).

[60] S. Takács, F. T. Tárkányi, M. Sonck, and A. Hermanne, New cross-sections and intercomparison of proton monitor reactions on $\mathrm{Ti}, \mathrm{Ni}$ and $\mathrm{Cu}$, Nuclear Instruments and Methods in Physics Research B 188, 106 (2002).

[61] S. J. Mills, G. F. Steyn, and F. M. Nortier, Experimental and Theoretical Excitation Functions of Radionuclides Produced in Proton Bombardment of Copper up to 200 $\mathrm{MeV}$, International Journal of Radiation Applications and Instrumentation Part A 43, 1019 (1992).

[62] A. Grütter, Excitation Functions for Radioactive Isotopes Produced by Proton Bombardment of $\mathrm{Cu}$ and $\mathrm{Al}$ in the Energy Range of 16 to $70 \mathrm{MeV}$, Nuclear Physics A 383, 98 (1982).

[63] H. R. Heydegger, C. K. Garrett, and A. Van Ginneken, Thin-Target Cross Sections for Some Cr, Mn, Fe, Co, $\mathrm{Ni}$, and Zn Nuclides Produced in Copper by 82- to 416MeV Protons, Physical Review C 6, 1235 (1972).

[64] C. J. Orth, B. J. Dropesky, R. A. Williams, G. C. Giesler, and J. Hudis, Pion-induced spallation of copper across the $(3,3)$ resonance, Physical Review C 18, 1426 (1978).

[65] H. Yashima, Y. Uwamino, H. Iwase, H. Sugita, T. Nakamura, S. Ito, and A. Fukumura, Measurement and calculation of radioactivities of spallation products by highenergy heavy ions, Radiochimica Acta 91, 689 (2003).

[66] L. R. Greenwood and R. K. Smither, Measurement of Cu spallation cross sections at IPNS, Tech. Rep. (U.S. Dept. of Energy, Fusion Energy Series, No.0046-18, 1984).

[67] I. R. Williams and C. B. Fulmer, Excitation Functions for Radioactive Isotopes Produced by Protons Below 60 $\mathrm{MeV}$ on $\mathrm{Al}, \mathrm{Fe}$, and $\mathrm{Cu}$, Physical Review 162, 1055 (1967).

[68] M. Shahid, K. Kim, H. Naik, M. Zaman, S. C. Yang, and G. Kim, Measurement of excitation functions in proton induced reactions on natural copper from their threshold to $43 \mathrm{MeV}$, Nuclear Instruments and Methods in Physics Research B 342, 305 (2015).

[69] M. S. Uddin, M. Hagiwara, F. T. Tárkányi, F. Ditrói, and M. Baba, Experimental studies on the protoninduced activation reactions of molybdenum in the energy range 22-67 MeV, Applied Radiation and Isotopes 60, 911 (2004).
[70] A. Hermanne, F. Szelecsenyi, M. Sonck, S. Takács, F. T. Tárkányi, and P. Van den Winkel, New cross section data on ${ }^{68} \mathrm{Zn}(\mathrm{p}, 2 \mathrm{n}){ }^{67} \mathrm{Ga}$ and ${ }^{n a t} \mathrm{Zn}(\mathrm{p}, \mathrm{xn}){ }^{67} \mathrm{Ga}$ nuclear reactions for the development of a reference data base, Journal of Radioanalytical and Nuclear Chemistry 240, 623 (1999).

[71] P. Kopecky, Proton Beam Monitoring via the $\mathrm{Cu}(\mathrm{p}, \mathrm{x}){ }^{58} \mathrm{Co},{ }^{63} \mathrm{Cu}(\mathrm{p}, 2 \mathrm{n}){ }^{62} \mathrm{Zn}$ and ${ }^{65} \mathrm{Cu}(\mathrm{p}, \mathrm{n}){ }^{65} \mathrm{Zn}$ Reactions in Copper, International Journal of Applied Radiation and Isotopes 36, 657 (1985).

[72] E. Z. Buthelezi, F. M. Nortier, and I. W. Schroeder, Excitation functions for the production of ${ }^{82} \mathrm{Sr}$ by proton bombardment of ${ }^{n a t} \mathrm{Rb}$ at energies up to $100 \mathrm{MeV}$, Applied Radiation and Isotopes 64, 915 (2006).

[73] M. U. Khandaker, M. S. Uddin, K. S. Kim, Y. S. Lee, and G. N. Kim, Measurement of cross-sections for the $(\mathrm{p}, \mathrm{xn})$ reactions in natural molybdenum, Nuclear Instruments and Methods in Physics Research B 262, 171 (2007).

[74] Y. Yoshizawa, H. Noma, T. Horiguchi, T. Katoh, S. Amemiya, M. Itoh, K. Hisatake, M. Sekikawa, and K. Chida, Isotope Separator On-Line at INS FM Cyclotron, Nuclear Instruments and Methods 134, 93 (1976).

[75] F. S. Al-Saleh, A. A. Al-Harbi, and A. Azzam, Excitation functions of proton induced nuclear reactions on natural copper using a medium-sized cyclotron, Radiochimica Acta 94, 391 (2006).

[76] Y. Titarenko, V. F. Batyaev, V. M. Zhivun, A. B. Koldobsky, Y. Trebukhovsky, E. I. Karpikhin, R. D. Mulambetov, S. V. Mulambetova, Y. Nekrasov, A. Titarenko, K. A. Lipatov, B. Sharkov, A. A. Golubev, A. D. Fertman, V. I. Turtikov, A. V. Kantsyrev, I. V. Roudskoy, G. N. Smirnov, V. S. Barashenkov, K. K. Gudima, M. I. Baznat, S. G. Mashnik, and R. E. Prael, Nuclide Production Cross Sections for ${ }^{59} \mathrm{Co}$ and ${ }^{n a t} \mathrm{Cu}$ Irradiated with $0.2 \mathrm{GeV}$ and $2.6 \mathrm{GeV}$ Protons and $0.2 \mathrm{GeV} /$ Nucleon Carbon Ions, in AccApp'03 Meeting (San Diego, California, 2003) p. 8.

[77] V. N. Aleksandrov, M. P. Semenova, and V. G. Semenov, Production Cross Section of Radionuclies in $(\mathrm{p}, \mathrm{x})$ Reaction at Copper and Nickel Nuclei, Soviet Atomic Energy 62, 411 (1987).

[78] J. Kuhnhenn, U. Herpers, W. Glasser, R. Michel, P. W. Kubik, and M. Suter, Thin target cross sections for proton-induced formation of radionuclides from lead for $E_{p} \leq 71 \mathrm{MeV}$, Radiochimica Acta 89, 697 (2001).

[79] C. U. Jost, J. R. Griswold, S. H. Bruffey, S. Mirzadeh, D. W. Stracener, and C. L. Williams, Measurement of cross sections for the ${ }^{232} \mathrm{Th}(\mathrm{p}, 4 \mathrm{n}){ }^{229} \mathrm{~Pa}$ reaction at low proton energies, AIP Conference Proceedings 1525, 10.1063/1.4802383 (2013).

[80] H. Junde, H. Xiaolong, and J. K. Tuli, Nuclear Data Sheets for A =67, Nuclear Data Sheets 106, 159 (2005).

[81] B. Singh and J. Chen, Nuclear data sheets for A $=73$, Nuclear Data Sheets 158, 1 (2019).

[82] S. M. Qaim and I. Spahn, Development of novel radionuclides for medical applications, Journal of Labelled Compounds and Radiopharmaceuticals 61, 126 (2018).

[83] J. Chen, B. Singh, and J. A. Cameron, Nuclear Data Sheets for $\mathrm{A}=44$, Nuclear Data Sheets 112, 2357 (2011).

[84] I. F. Chaple and S. E. Lapi, Production and use of first row transition metal PET radionuclides, ${ }^{43,44} \mathrm{Sc},{ }^{52} \mathrm{Mn}$, 
and ${ }^{45} \mathrm{Ti}$, Journal of Nuclear Medicine 59, 1655 (2018).

[85] S. Ferguson, H. Jans, M. Wuest, T. Riauka, and F. Wuest, Comparison of scandium- $44 \mathrm{~g}$ with other PET radionuclides in pre-clinical PET phantom imaging, European Journal of Nuclear Medicine and Molecular Imaging Physics 6, 1 (2019).

[86] Y. Nagame, H. Nakahara, and M. Furukawa, Excitation Functions for $\alpha$ and ${ }^{3} \mathrm{He}$ Particles Induced Reactions on Zinc, Radiochimica Acta 46, 5 (1989).

[87] F. H. Ruddy and B. D. Pate, Formation and decay of the compound nucleus ${ }^{68} \mathrm{Ge}$, Nuclear Physics A 127, 305 (1969).

[88] N. T. Porile, S. Tanaka, H. Amano, M. Furukawa, S. Iwata, and M. Yagi, Nuclear Reactions of $\mathrm{Ga}^{69}$ and $\mathrm{Ga}^{71}$ with 13-56 MeV Protons, Nuclear Physics 43, 500 (1963).

[89] S. Amiel, Reactions of Alpha Particles with Germanium-70 and Zinc-70, Physical Review 116, 415 (1959).

[90] D. D. Villiers, M. Nortier, and W. Richter, Experimental and theoretical excitation functions for ${ }^{\text {nat }} \operatorname{Br}(\mathrm{p}, \mathrm{x})$ reactions, Applied Radiation and Isotopes 57, 907 (2002).

[91] R. Adam-Rebeles, A. Hermanne, P. Van Den Winkel, L. De Vis, R. Waegeneer, F. Tárkányi, S. Takács, and M. P. Takács, ${ }^{68} \mathrm{Ge} /{ }^{68} \mathrm{Ga}$ production revisited: Excitation curves, target preparation and chemical separation - purification, Radiochimica Acta 101, 481 (2013).

[92] A. Hermanne, R. Adam-Rebeles, F. Tárkányi, S. Takács, and F. Ditrói, Proton and deuteron induced reactions on ${ }^{n a t} \mathrm{Ga}$ : Experimental and calculated excitation functions, Nuclear Instruments and Methods in Physics Research B 359, 145 (2015).

[93] A. Mushtaq and S. M. Qaim, Excitation Functions of $\alpha$ and ${ }^{3} \mathrm{He}-\mathrm{Particle}$ Induced Nuclear Reactions on Natural Germanium: Evaluation of Production Routes for ${ }^{73} \mathrm{Se}$, Radiochimica Acta 50, 27 (1990).

[94] B. Effenberger, W. Kunold, W. Oesterle, M. Schneider, L. M. Simons, R. Abela, and J. Wüest, Determination of the spectroscopic quadrupole moments of ${ }^{75} \mathrm{As}_{33}$ and ${ }^{63} \mathrm{Cu}_{29}$, Zeitschrift fur Physik A - Atoms and Nuclei 309, 77 (1982).

[95] P. Verma, C. Sharma, S. Singh, A. Bharti, and S. K. Khosa, Theoretical Overview of Back-bending in Arsenic Isotopes, AIP Conference Proceedings 1524, 101 (2013).

[96] S. Goriely, M. Samyn, and J. M. Pearson, Further explorations of Skyrme-Hartree-Fock-Bogoliubov mass formulas. VII. Simultaneous fits to masses and fission barriers, Physical Review C 75, 064312 (2007).

[97] C. G. Li, Q. B. Chen, S. Q. Zhang, C. Xu, H. Hua, X. Q. Li, X. G. Wu, S. P. Hu, J. Meng, F. R. Xu, W. Y. Liang, Z. H. Li, Y. L. Ye, D. X. Jiang, J. J. Sun, R. Han, C. Y. Niu, X. C. Chen, P. J. Li, C. G. Wang, H. Y. Wu, G. S. Li, C. Y. He, Y. Zheng, C. B. Li, Q. M. Chen, J. Zhong, and W. K. Zhou, Observation of a novel stapler band in ${ }^{75}$ As, Physics Letters B 766, 107 (2017).

[98] S. M. Grimes, Hauser-Feshbach calculations in deformed nuclei, Physical Review C 88, 24613 (2013).

[99] R. Capote, M. Herman, P. Oblozinsky, P. G. Young, S. Goriely, T. Belgya, A. V. Ignatyuk, A. J. Koning, S. Hilaire, and Others, RIPL - Reference Input Parameter Library for Calculation of Nuclear Reactions and Nuclear Data Evaluations, Nuclear Data Sheets 110,
3107 (2009)

[100] A. Obertelli, T. Baugher, D. Bazin, S. Boissinot, J. P. Delaroche, A. Dijon, F. Flavigny, A. Gade, M. Girod, T. Glasmacher, G. F. Grinyer, W. Korten, J. Ljungvall, S. McDaniel, A. Ratkiewicz, B. Sulignano, P. Van Isacker, and D. Weisshaar, First spectroscopy of ${ }^{66} \mathrm{Se}$ and ${ }^{65}$ As: Investigating shape coexistence beyond the $\mathrm{N}=\mathrm{Z}$ line, Physics Letters B 701, 417 (2011).

[101] A. M. Bruce, J. Simpson, D. D. Warner, C. Baktash, C. J. Barton, M. A. Bentley, M. J. Brinkman, R. A. Cunningham, E. Dragulescu, L. Frankland, T. N. Ginter, C. J. Gross, R. C. Lemmon, B. MacDonald, C. D. O'Leary, S. M. Vincent, R. Wyss, C. H. Yu, and N. V. Zamfir, Two-neutron alignment and shape changes in ${ }^{69}$ As, Physical Review C 62, 027303 (2000).

[102] T. Kawano, New Arsenic Cross Section Calculations, Tech. Rep. (LA-UR-15-21585, Los Alamos National Laboratory, Nuclear Data Team, Los Alamos, NM, 2015).

[103] K. Shibata, G. Chiba, A. Ichihara, and S. Kunieda, Evaluation of Neutron Nuclear Data on Arsenic-75 for JENDL-4, Journal of Nuclear Science and Technology 47, 40 (2012).

[104] A. Negret and B. Singh, Nuclear data sheets for A = 75, Nuclear Data Sheets 114, 841 (2013).

[105] B. Singh and A. R. Farhan, Nuclear Data Sheets for A = 74, Nuclear Data Sheets 107, 1923 (2006).

[106] B. Singh, Nuclear Data Sheets Update for A = 76, Nuclear Data Sheets 74, 63 (1995).

[107] A. D. Ayangeakaa, R. V. Janssens, S. Zhu, D. Little, J. Henderson, C. Y. Wu, D. J. Hartley, M. Albers, K. Auranen, B. Bucher, M. P. Carpenter, P. Chowdhury, D. Cline, H. L. Crawford, P. Fallon, A. M. Forney, A. Gade, A. B. Hayes, F. G. Kondev, Krishichayan, T. Lauritsen, J. Li, A. O. MacChiavelli, D. Rhodes, D. Seweryniak, S. M. Stolze, W. B. Walters, and J. Wu, Evidence for Rigid Triaxial Deformation in ${ }^{76} \mathrm{Ge}$ from a Model-Independent Analysis, Physical Review Letters 123, 102501 (2019).

[108] S. Goriely, F. Tondeur, and J. M. Pearson, A HartreeFock Nuclear Mass Table, Atomic Data and Nuclear Data Tables 77, 311 (2001).

[109] S. Hilaire, M. Girod, S. Goriely, and A. J. Koning, Temperature-dependent combinatorial level densities with the D1M Gogny force, Physical Review C 86, 064317 (2012).

[110] A. J. Koning and J. P. Delaroche, Local and global nucleon optical models from $1 \mathrm{keV}$ to $200 \mathrm{MeV}$, Nuclear Physics A 713, 231 (2003).

[111] V. Avrigeanu, M. Avrigeanu, and C. Manailescu, Further explorations of the $\alpha$-particle optical model potential at low energies for the mass range $A \approx 45-209$, Physical Review C 90, 44612 (2014).

[112] Y. Han, Y. Shi, and Q. Shen, Deuteron global optical model potential for energies up to $200 \mathrm{MeV}$, Physical Review C 74, 044615 (2006).

[113] S. Watanabe, High energy scattering of deuterons by complex nuclei, Nuclear Physics 8, 484 (1958).

[114] G. P. Nobre, D. A. Brown, M. W. Herman, and A. Golas, Constraining level densities through quantitative correlations with cross-section data, Physical Review C 101, 1 (2020).

[115] T. Kawano, S. Chiba, and H. Koura, Phenomenological Nuclear Level Densities using the KTUY05 Nuclear 
Mass Formula for Applications Off-Stability, Journal of Nuclear Science and Technology 43, 1 (2006).

[116] S. M. Grimes, T. N. Massey, B. M. Oginni, S. Shukla, and A. Voinov, Nuclear Level Densities Off of the Stability Line, AIP Conference Proceedings 1005, 57 (2008).

[117] P. Roy, K. Banerjee, T. K. Rana, S. Kundu, S. Manna, A. Sen, D. Mondal, J. Sadhukhan, M. T. Senthil Kannan, T. K. Ghosh, S. Mukhopadhyay, D. Pandit, G. Mukherjee, S. Pal, D. Paul, K. Atreya, and C. Bhattacharya, Evidence for the reduction of nuclear level density away from the $\beta$-stability line, Physical Review C 102, 061601(R) (2020).

[118] S. M. Grimes, J. D. Anderson, A. K. Kerman, and C. Wong, Role of Isospin in Statistical Processes, Physical Review C 5, 85 (1972).

[119] A. J. Koning and M. C. Duijvestijn, A global preequilibrium analysis from 7 to $200 \mathrm{MeV}$ based on the op- tical model potential, Nuclear Physics A 744, 15 (2004).

[120] E. Vagena, M. Axiotis, and P. Dimitriou, Systematics of semi-microscopic proton-nucleus optical potential at low energies relevant to nuclear astrophysics, arXiv (2021), arXiv:2103.07129.

[121] C. Romano, L. A. Bernstein, T. Bailey, F. Bostelmann, D. Brown, Y. Danon, R. Casperson, J. Conlin, M. Devlin, B. Goldblum, M. Grosskopf, A. Lewis, D. Neudecker, E. M. O'Brien, B. Pierson, B. Quiter, A. Ratkiewicz, G. W. Severin, M. Smith, V. Sobes, A. Sonzogni, P. Talou, F. Tovesson, E. Vermeulen, K. Wendt, and M. Zerkle, Proceedings of the Workshop for Applied Nuclear Data: WANDA2020, Tech. Rep. (ORNL/TM-2020/1617, Oak Ridge National Laboratory, Washington, D.C., 2020).

[122] M. B. Fox, TREND As, Nb, Cu, Ti Gamma Spectroscopy [Data set] (2021). 